POINT-CONTACT SPECTROSCOPY

IN METALS

A.G.M. JANSEN 



\section{POINT-CONTACT SPECTROSCOPY}

\section{IN METALS}


PROMOTOR

PROF. DR. P. WYDER

CO -REFERENT

DR. A P van GELDER 


\title{
POINT-CONTACT SPECTROSCOPY \\ IN METALS
}

\author{
P R O E F S C H R I F T
}

TER VERKRIJGING VAN DE GRAAD VAN DOCTOR IN DE WISKUNDE EN NATUURWETENSCHAPPEN

AAN DE KATHOLIEKE UNIVERSITEIT TE NIJMEGEN OP GEZAG VAN DE RECTOR MAGNIFICUS PROF. DR. P.G.A.B. WIJDEVELD, VOLGENS BESLUIT VAN HET COLLEGE VAN DECANEN

IN HET OPENBAAR TE VERDEDIGEN

OP DONDERDAG 24 APRIL 1980

DES NAMIDDAGS TE 2.00 UUR PRECIES

door

ALOYSIUS GERARDUS MARIA JANSEN

geboren te Breda

1980

Druk: Krips Repro Meppel 
Iedereen, die op enigerlel wijze een bijdrage heeft geleverd aan het tot stand komen van dit proefschrift wil ik hiervoor hartelıjk bedanken. In het bijzonder Rikl Gommers en Lies Remers voor de verzorging van de lay-out, de medewerkers van de dienstverlenende afdelingen van de facultelt der wiskunde en natuurwetenschappen en niet in de laatste plaats alle medewerkers van de afdelıng experımentele natuurkunde 4 .

Dit onderzoek werd ultgevoerd op de afdeling experımentele natuurkunde 4 van het Research Instituut voor Materlalen van de Katholıeke Unıversitelt te Nljmegen, onder lelding van Prof. Dr. P. Wyder. Een gedeelte van dit onderzoek is gesteund door de Stichting voor Fundamenteel onderzoek der Materıe (FOM) met financiele bijdragen van de Nederlandse Organısatıe voor Zuıver Wetenschappelıjk Onderzoek (zWO).

We acknowledge the permission to reprint previously published papers obtalned from the publishers to The Physical Revicw, Sclence and Journal de Physique. 
Voor minn ouders

Uoor Maryte 
Chapter I GENERAL INTRODUCTION 1

References 6

Chapter II POINT-CONTACT SPECTROSCOPY IN METALS 7

1. Introduction 8

2. Methods for obtaining phonon spectra 10

3. Constriction resistance and its voltage dependence 12

4. Solution of the Boltzmann equation

4.1 Formulation of the problem 18

4.2 Solution in zeroth order: Sharvin current 21

4.3 Solution in first order: backflow current 27

4.4 Application to the electron-phonon interaction 30

4.5 Background signal 35

5. Experimental techniques

5.1 Point-contact fabrication 39

5.2 Electronics for recording derivatives 40

6. Experimental results 43

7. Temperature dependence of point-contact spectroscopy 58

8. Detection of other scattering mechanisms than the electron-phonon interaction 64

References $\quad 73$

Chapter III DIRECT MEASUREMENT OF ELECTRON-PHONON COUPLING $\alpha^{2} F(\omega)$ USING POINT CONTACTS: NOBLE METALS 76

Chapter IV NORMAL METALLIC POINT CONTACTS

Chapter V STRUCTURE OF CURRENT-VOLTAGE CHARACTERISTICS OF METAL POINT CONTACTS 84

Chapter VI POINT-CONTACT SPECTROSCOPY IN NORMAL METALS 86

References $\quad 95$

Chapter VII TEMPERATURE DEPENDENCE OF POINT-CONTACT SPECTROSCOPY $\begin{array}{ll}\text { IN COPPER } & 96\end{array}$

$\begin{array}{lr}\text { References } & 114\end{array}$ 
Chapter VIII APPLICATION OF POINT-CONTACT SPECTROSCOPY IN METALS TO THE KONDO PROBLEM 

Chapter I : GENERAL INTRODUCTION

It is one of the fascinating aspects of the quantum theory of solids that in a metal, the electrons can move freely over a farly large distance, called the mean free path (up to $1 \mathrm{~cm}$ in pure metals at low temperatures), without a collision with, for instance, impurities or lattice vibrations (phonons). In the absence of an applied voltage over the metal, the electrons move in random directions and no net current flows in the sample. In a uniform electric field, caused by an applied voltage, the electrons are accelerated over a distance of the order of the mean free path in the direction of the field and the electrons get a preferred velocity-component, the drift velocity, which is responsible for the current through the metal. After a collision, the electron comes back in equilibrium, and then the acceleration process starts again. Using this simple mean-free-path model for the electrical conduction, one can readıly show that the applied voltage over a metal sample is proportional to the electrical current. This is the well known Ohm's law. Due to the high electrical conductivity of a metal, the drift velocity is very small compared to the average random velocity of the electrons (Fermi velocity). This is why Ohm's law is such a good approximation for the relation between the current and the voltage in a metal. Increasing the voltage will certainly increase the drift velocity of the electrons, but from an experimental point of view, again due to the high electrical conductivity, deviations from Ohm's law are difficult to distinguish from ordinary heating effects.

In this thesis, we discuss interesting non- linear phenomena observed in the current-voltage characteristics of metallic constrictions (point contacts) whlch have a linear dimension a comparable to or smaller than the electronic mean free path $l(a<\ell)$. In these point contacts, it is possible to have a rather high electric field within a metal, impossible otherwise, wich gives the electrons a considerable drift velocity within the metal by simply applying a voltage over the contact. No significant heating occurs in this geometry. The observed non-linearlty of the currentvoltage relation in metallic point contacts can be used for a fundamental study of the interactions of the electrons in a metal. A spectral analysis of the relevant scattering mechanisms for the conduction electrons in a metal is possible. This experimental method, developed in Prof. I.K.Yanson's 
laboratory in Kharkov and in our laboratory in Nijmegen is nowadays called "polnt-contact spectroscopy".

Starting with the advent of rotating machinery at the end of the $19 \mathrm{th}$ century, the problem of the understanding of electrical contacts is qute old. Today, in the technology of micro electronics, the manufacturing of metalilc contacts is again important in order to attach stable metallic leads to semi-conducting devices (diodes, transistors, integrated circults). The current densities in contacts of these small-scale devices can be as large as $10^{6} \mathrm{~A} / \mathrm{cm}^{2}$ (a few milliampères through a contact with a linear dimension of a few microns). The point contacts under study in pointcontact spectroscopy and discussed in this thesis have even larger current densities (up to $10^{11} \mathrm{~A} / \mathrm{cm}^{2}$ ) and reveal remarkable non-linear behaviour. The first ploneering experiments in this rather new fleld were done by Yanson ${ }^{1}$ in Kharkov (USSR) in a study of the current-voltage characteristics of tunnel junctions (two metal films separated by an oxide layer) with a short circult between the metal films deliberately or accidentally produced. He discovered that the contact resistance of these micro contacts at liquid helium temperatures increased remarkably in a non-linear way at applied voltages which correspond to the phonon frequencies of the bulk metal forming the junction. This observation indicates that the non-linearity is related to the coupling between the conduction electrons and the phonons. Moreover, Yanson found, as the grand result for pointcontact spectroscopy, that the measured voltage derivative of the resistance gives a signal as function of the applied voltage which is proportional to the electron-phonon coupling parameter $\alpha^{2} F(\omega) \cdot \alpha^{2} F(\omega)$ characterlzes the energy dependent electron-phonon interaction, loosely speaking, F is the phonon density of states and $\alpha$ the matrix element for the electronphonon interaction averaged over the Fermi sphere.

The applicability of this spectroscopic method has been dramatically increased by the discovery that also metallic point contacts, consisting of a sharply etched needle ("spear") pressed agannst a bulk surface ("anvil"), are useful for an experımental study of the phenomenum ${ }^{2}$. These pressure-type point contacts make it possible to use the method in a simple and convenient way for an experimental study of a large varlety of samples, including single crystals in order to study anısotropy effects. In figure 1 we give an example of the current-voltage characteristics for a pressure-type point contact of copper. The increase in the resistance 


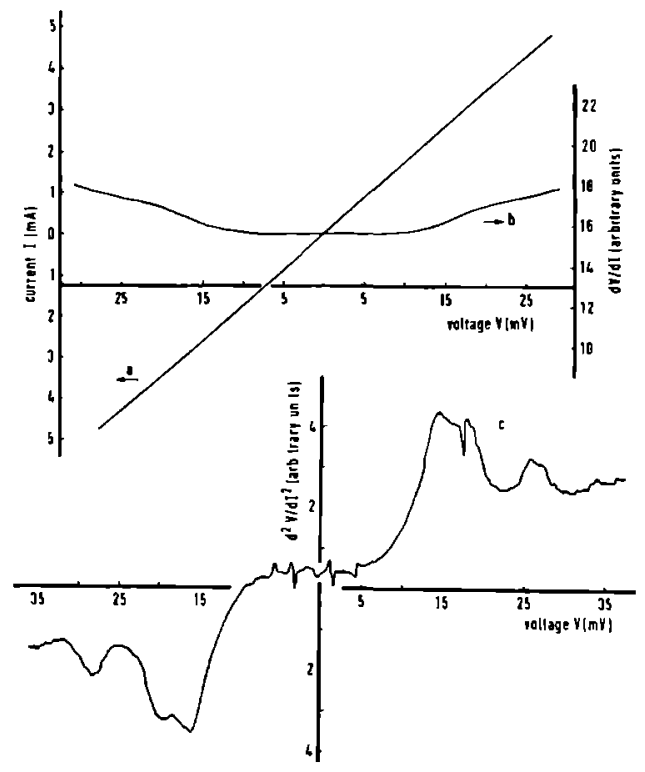

Fig. 1 Current-voltage characteristics as directly neasured on a recorder of a copper point contact with a resistance $R_{0}=5.7 \Omega$ at a temperature $T=1.2 \mathrm{~K}$. The current $I$ (a), the differential resistance $d V / d I(b)$ and the second derivative $d^{2} v / d I^{2}$ (c) are glven as a function of the applied voltage $V$. The structures in the $\mathrm{d}^{2} \mathrm{v} / \mathrm{dI}^{2}-$ signal around 17 and $27 \mathrm{mV}$ correspond to the bulk phonon frequencles of copper.

$\mathrm{dV} / \mathrm{dI}$ around 17 and $27 \mathrm{mV}$ and the resulting structures in the $\mathrm{d}^{2} \mathrm{v} / \mathrm{dI} \mathrm{I}^{2}$ signal are clearly visible. These experiments reveal in a very clear way the $\alpha^{2}$ F-spectrum for the electron-phonon interaction in copper. The coupling between electrons and phonons plays an important role in many physical properties of metals, such as the electrical conductivity, the thermal conductivity, superconductivity etc. This makes that an experimental determination of the function $a^{2} F$ is of great interest. This is of particular importance, as theoretical band structure calculations of $\alpha^{2} F$ seem to be rather difficult and not too reliable. The point-contact method gives, as an example, detalled information about the relative strength of the coupling of the electrons with the various phonon modes (transverse and longıtudinal) in a metal. For normal metals, (1.e non-superconductors), point-contact spectroscopy 1s, at 
least until now, the only experimental method which can detormine the energy dependence of the function $\alpha^{2} F$ successfully.

For an intultive explanation of the non- linearity in the currentvoltage characteristic of a point contact it is instructive to make a side step to one of the classic experiment in atomic pnysics: the Franck-Hertz experiment ${ }^{3}$. In a tube filled with a dilute gas of mercury atoms, electrons are accelerated through a potential. Increasing the potential results in an increase of the kinetic energy of the electrons. If the kinetic energy of the electrons reaches the quantum energy, whlch can be absorbed by a Hg atom, the conductivity of the gas chamber decreases. This process results in the periodic pattern of the conductivity of a dilute gas of mercury atoms in an applied electric field,as shown in figure 2. The Franck-liertz experiment sjows in a clear way that the emission and absorption of light is quantized, and, more important in our context, that the scattering cross section for the scattering of the electrons with $\mathrm{Hg}$ atoms is a measurable function of the energy. For a point contact between two metals, a simular situation holds: The electrons are accelerated in the electric field caused by the applied voltage by passing the contact area. A moderation and a slow down of the injected electrons occurs via inelastic collisions with phonons, these processes depend strongly on the energy of the electrons and give rise to a non-linear current-voltage characterıstıc due to the electronphonon interaction.

In a theoretical analysis 4,5 , the transport problen for the electrical current througn a point contact in the limit a<l has been solved, using the concept of "blackflow". After having suffered an inelastic collısion (spontaneous emission of the phonon) the electrons which have been injected through the contact, can flow back through the constriction. This explains the increase in resistance at typical phonon energies. The theoretical analysis shows that the second derıvatıve signal for a metallic point contact is proportional to a slight modification of the usual function $\alpha^{2} \mathrm{~F}$. The modiflcation has to do with the txansport efficiency for the occurence of a blackflow process in the point- contact geometry.

Until now point-contact spectroscopy has been used almost exclusively to study the electron-phonon interaction. It is obviously very much worthwle to investigate the possibility if other scatterers than phonons can be probed via the same point-contact method. Recently, the interaction of electrons with magnons ${ }^{6}$ and with paramagnetic 1 mpurities ${ }^{7}$ has been 


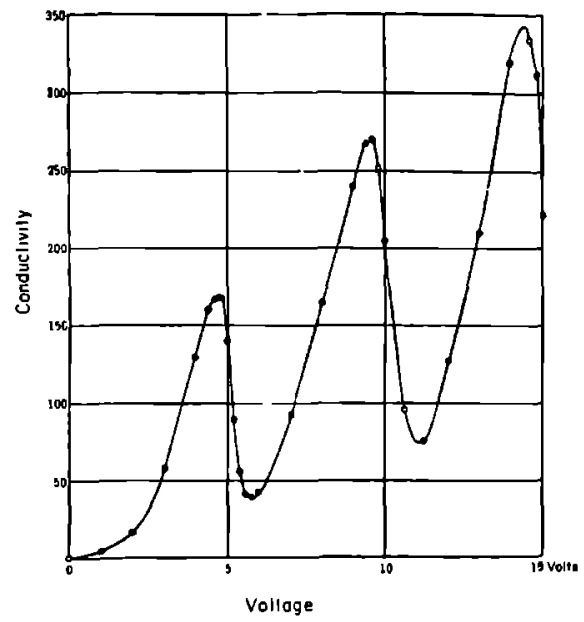

Fig. 2 The Franck-Hertz experiment from atomic physics. The conductivity $\mathrm{dI} / \mathrm{dV}$ as a function of the voltage in a dilute gas of mercury atoms (After the orıginal paper by Franck and Hertz).

detected successfully in point- contact experiments, with, respectively, ferromagnetic metals and magnetıcally dilute alloys (Kondo systems). These experiments give a promising future for the detection of all kinds of scattering mechanisms of the conduction electrons in a metal using point-contact spectroscopy. The only limiting condition seens to be that the electronic mean free path of the sample to be investigated in a pointcontact geometry has to be comparable or larger than the dimensions of the contact.

The thesis is organized as follows. In the next chapter, a survey is given of the theoretical and experimental work done in this field with its exciting "sharp points". After this review, the following chapters treat several aspects in point-contact experiments, involving the measurement of the electron-phonon coupling in noble metals, the observation of double phonon processes, the temperature dependence of the spectroscopic method and the application of point-contact experiments to Kondo systems. All chapters are given in the form of published papers or papers to be publıshed. 
1. I.K. Yanson, Zh. Eksp. Teor. Fiz. 66, 1035 (1974) (Sov. Phys. - JETP 39, 506 (1974)\}.

2. A.G.M. Jansen, F.M. Mueller and P. Wyder, Proc. 2nd Rochester Conf. on Superconductivity in $d$ - and f-band Metals, ed. D.H. Douglass (Plenum, New York, 1976).

3. J. Franck and G. Hertz, Verhand. Deut. Physik. Ges. 16, 457 (1914); ibidem p. 512 .

4. I.O. Kulik, A.N. Omel'yanchuk and R.I. Shekhter, Fiz. Nizk. Temp. 3 , 1543 (1977) \{Sov. J. Low Temp. Phys. 3 , 740 (1977)\}.

5. A.P. van Gelder, Solid State Commun. 25, 1097 (1978).

6. B.I. Verkin, I.K. Yanson, I.O. Kulik, O.I. Shklyarevski, A.A. Lysykh and Yu.G. Naydyuk, Solid State Commun. 30, 215 (1979).

7. A.G.M. Jansen, A.P. van Gelder, P. Wyder and S. Strāssler, to be published; see chapter VIII. 


\section{Abstract}

Polnt contacts between normal metals at low temperatures show very interesting non-linear phenomena in the current-voltage characteristics. The observed deviations from Ohm's law in metallic constrictions can be used for an energy resolved spectral analysis of the interaction mechanisms of the conduction electrons with elementary excitations in a metal. A review 15 given on the theoretical and experimental work dealing with this spectroscopic method. The metalilc point contacts investigated show structures in the measured secord derivatıve $d^{2} V / d I^{2}$ which are in agreement with structures in the phonon density of states of the metal under study. A theoretical analysis, involving an iterative solution of the full non-linear Boltzmann equation for the transport problem of a current through a point-contact geometry, 15 able to show that the observed second derivative signal 15 proportional to Eliashbergs form of the electron-phonon interaction $\alpha^{2} F(\omega)$, with a slight modification due to a transport efficiency function. Non-equilibrium effects of the phonon system are discussed in relation to the observed background signal on which the $\alpha^{2}$ F-signal is thought to be superimposed. The theory can easily be extended to other scattering mechanisms than the electron-phonon interaction, and some experiments show that the interactions of conduction electrons with magnons and paramagnetic impurities can be detected using point contacts between ferromagnetic metals and between magnetically dilute alloys. 
1. Introduction

The problems of electrical contacts between metals have always attracted a great deal of interest. Especially in the development of micro electronics, much effort has been put in the production of reliable contacts to these small-scale devices. For the metallic contacts, one usually expects a linear relation between the applied voltage and the current through the contact, according to Ohm's law. However, interesting non-linear behaveour can be observed in the current-voltage characteristics of metallic constrictions if the linear dimension of the contact becomes comparable to or smaller than the mean free path of the electrons in the metal. It turns out that the observed phenomenum can be used for a fundamental study of the scattering mechanisms of the conduction electrons in a metal, leading to a new experimental tool, nowadays referred to as "polnt-contact spectroscopy". In this paper we want to give a review on the experimental and theoretical work done in this field following the first ploneering and inportant publication by Yanson (1974 a).

In an expcrimental study of metal-insulator-metal tunnel junctions, with a short circult in the oxide layer between the metal films, Yanson (1974 a) has found that the current-voltage characterlstics of these metallic micro contacts showed a non-linear behaviour at liquid helium temperatures. To characterıze the non-1ınearity Yanson has measured the second derivative $\mathrm{d}^{2} \mathrm{v} / \mathrm{dI}^{2}$ of the voltage with respect to the current as a function of the voltage $V$. As a most remarkable result, he observed structures in the measured second-derivative signal at applied voltages corresponding to the bulk phonon frequencies of the metal forming the junction. Yanson interpreted the experinental data as a direct measurement of the electronphonon interaction function $\alpha^{2}$ F. Roughly speaking, the Eliashberg function $\alpha^{2} F$ is the product of the phonon density of states $F$ and the squared matrıx element $\alpha$ for the electron-phonon interaction, averaged over the Fermi sphere. As the radius a of the investigated contacts is small compared to the mean free path $l$ of the electrons $(a<\ell)$, the transport of electrons through the contact is ballıstic and no longer diffuse. Within a mean free path distance, the electrons are accelerated due to the electric field caused by the applied voltage, and are injected from one metal side to the other by passing the contact (Sharvin, 1965). The accelerated electrons are brought back into equilibrium via inelastic collisions with, for 
instance, phonons. In paragraph 3 of this paper, we wlll show in a simplıfied and intultıve plcture that one can easily get an interpolation formula for the resistance of a contact in the intermediate regime between the IImiting cases of $a \gg l$ or $a \ll \ell$ and $1 t$ is found that the inelastic phonon emission near the orlfice ylelds a second derlvative signal which is proportional to the Elıashherg function $\alpha^{2} \mathrm{~F}$. Recently, theoretical work with a more fundamental approach has become avallable (paragraph 4). The full non-linear Boltzmann equation has been solved for the point-contact problem, Introducing the concept of "backflow" (Kulık et al., 1977; van Gelder, 1978). After injection, the electrons can flow back through the orlfice due to an inelastıc scattering process; thıs process gives rise to a negative and voltage-dependent correction to the current. Hence, the non-linear current-voltage relation contalns information about the inelastic collisions of the electrons. A careful analysis then shows that the measured spectrum is characterized by a slight modification of the function $\alpha^{2} F$, involving a geometrical transport efficiency for the backflow current arising from the electron-phonon interaction. In addition, in most pointcontact spectra a smooth background signal is observed, on which the $\alpha^{2}$ F-signal is thought to be superimposed. An explanation for the background signal can be glven in terms of a non-equilibrium distribution of the phonon system, whlch glves additional corrections to the current from stimulated emssion and absorption processes (van Gelder et al., 1978; van Gelder, 1980).

Experimentally, various metals have been investigated using point-contact spectroscopy for the measurement of the electron-phonon interaction (Yanson and Kulık, 1978). The method has been extended for the investıgatıon of a large varıety of samples, including single crystals, by the fact, that pressure-type polnt contacts, consısting of a sharply etched spear pressed agaınst a bulk anvil, appeared to be very useful for spectroscoplc investıgations (Jansen et al., 1977 and 1978). In paragraph 5, the experimental techniques used for the polnt-contact method will be discussed. Paragraph 6 contains a survey of the experimental results of the measured electronphonon interaction in metals using metallic point contacts. This technique provides interesting and novel results, especially for the normal metals, whlch have not been studied before. For instance, one gets direct experimental information concerning the relative strength of the various phonon modes (transverse or longitudinal) in the electron-phonon coupling. 
The influence of the temperature will be treated in paragraph 7. An increase of the measuring temperature results in a broadening of the measured second derivative spectra. It is very interesting to compare the temperature dependence of the point-contact resistance at zero voltage with that of the bulk resistivity. Small differences in the temperature dependence for the two cases can be ascribed to differences in the transport efficlency in the bulk and in the point-contact geometry (van Gelder et al., 1980 a).

It is obvious that point-contact spectroscopy can in principle be applied to any arbitrary scatterer, not only phonons. The relevant theory will therefore be presented accordingly. In paragraph 8 experimental results will be discussed which have been performed in ferromagnets (Verkin et al., 1979) and in magnetically dilute alloys (Jansen et al., 1980 b) in order to detect other scattering mechanisms than the electron-phonon interaction. In the point-contact experiments with ferromagnets, structure is observed which can be ascribed to strong heating in the vicinity of the metallıc contact. A modified temperature dependent resıstivity is measured arising from electron-magnon interaction. The experiments with the Kondo systems seem to open the possibility for a direct determination of the energy-dependent scatterıng time for a metal containing paramagnetıc $1 \mathrm{~m}-$ purities from the change in resistance of a point-contact.

However, a word of caution should be sald about the general applicablilty of point-contact spectroscopy as a general tool to measure the energy dependence of the scattering length. As the technique works only if the dimensions of the polnt contact are small or at least comparable to the mean free path of the electrons, point-contact spectroscopy can only be applied successfully to reasonably pure metals with not too short a mean free path.

2. Methods for obtalning phonon spectra

It is rather difficult to determine theoretically the Eliashberg function $\alpha^{2} F$, responsible for the electron-phonon interaction in metals. Therefore, it is of considerable importance to measure the phonon spectra experimentally; this can be done in several ways. Detalled phonon dispersion curves $w(q)$ (phonon frequency $w$ as function of wave number $q$ ) along the principal symmetry directions of a single crystal are obtained from 
experiments with coherent inelastic neutron scattering (Dolling and Woods, 1965); these dispersion curves can then be fitted to an interatomic force model for nearest nelghbor interactions (Born-von Karman analysis). By sampling over a large number of q-values, the phonon density of states $F(w)$ is then calculated from the dispersion curves. For materials, which absorb neutrons (1.e. ${ }^{3}$ He or $\mathrm{cd}$ ), diffuse $\mathrm{x}$-ray scatterıng can be used in a similar way to determine the phonon dispersion curves leading to $F(\omega)$ (Walker, 1956).

Using the superconducting tunneling mechanism in a geometry of two metal films separated by an oxıde layer the electron-phonon interaction $\alpha^{2} \mathrm{~F}$ can be measured for superconducting metals (McMlllan and Rowel1, 1969). The density of states in a superconductor is obtalned experimentally from the conductivity of the tunnel junction in the superconducting state, normalized to that in the normal state. Theoretically, using the theory of strong coupling superconductors (Parks, 1969), the density of states can be found by an iterative solution of the coupled Eliashberg equations containing the function $\alpha^{2}$ F. By trial and error, it is then possible to determine $\alpha^{2} \mathrm{~F}$ by comparing the theoretically calculated density of states with the experimental results. Using the proximity effect, in a tunnel junction phonon structures have been observed in a normal metal backed by a superconductor (Chalkın and Hansma, 1976).

In tunneling experiments with superconductors put in the normal state by a magnetic field, non-linearities in the current-voltage characteristics have been observed at typical phonon energies (Rowell et al., 1969), whlch can be analysed in terms of an enhanced tunneling probability arising from phonon modes of the surface layer in the metal electrodes. It is interesting to note that in these tunneling experiments an increase is seen in the conductivity of the junction, where polnt-contact spectroscopy shows an $1 n-$ crease in the contact resistance.

In Mossbauer experiments (Frauenfelder, 1962) a moment of the function $\alpha^{2} F\left(f \alpha^{2} F(\omega) \omega^{n} d \omega\right.$ for $\left.n= \pm 1, \pm 2\right)$ can be measured. One should realize that the energy dependence of the electron-phonon interaction is also contained in the temperature dependence of the electrical resistivity, which could allow a determination of $\alpha^{2} F$ using a suitable analysis. Polnt-contact spectroscopy seems to be an important tool to measure the function $\alpha^{2}$ F. Especially for non-superconducting metals, it is the only known method to yield detalled information on the energy dependence 
of the electron-phonon interaction. As up to now phonon spectra of metals are most elaborately studied usıng inelastıc neutron scattering (F( $\omega)$ ) and superconducting tunneling-spectroscopy $\left(\alpha^{2} F(\omega)\right)$, we wlll compare the pointcontact spectra with spectra from these experimental techniques.

3. Constriction resistance and its voltage dependence

We want to describe the voltage dependence of the electrical resistance of a metallic point contact at low temperatures which reveals the non-linearity in the current-voltage characteristics arising from inelastic scattering processes of the electrons. In this paxagraph, we use a heuristic approach in order to clarify the role of the relavant parameters in pointcontact experiments. In particular we discuss in detall the interactions of the electrons with the lattice vibrations, 1.e. the electron-phonon interaction. In paragraph 4 a more formal solution of the non-linear Boltzmann equation for the polnt-contact problem will be discussed; this approach will be extended to arbltrary targets for inelastic scatterıng of the conduction electrons.

The interest in the understanding of electrical contacts, often called the problem of the "constriction resistance" is quite old (Holn, 1967). Already Maxwell (1904) has studied the problem of the resistance of a small metallic contact. He solved in a classical way Polsson's equation for the electrical potential energy $\phi$ as a function of the position $\underline{r}$

$$
v^{2} \phi(\underline{r})=0
$$

in oblate spherical coordinates. For a current flowing through a constricting circular orifice of radius a the contours of constant potential energy are given by

$$
\phi(\underline{r})= \pm \frac{\mathrm{eV}}{2}\left[1-\frac{2}{\pi} \arctan (1 / \xi)\right],
$$

where the oblate spherical coordinates may be found from the more familiar spherical coordinates $r$ and $z$ by solving the lmplicit equation $r^{2} / a^{2}=$ $\left(1+\xi^{2}\right)\left(1-z^{2} / \xi^{2} a^{2}\right)$ (figure 1). The two signs refer to the two sides of the contact. The total voltage $v$, applied over the contact, devided by the current through any contour of constant potential yield an expression for the contact resistance. For simplicity we choose the contour with $\xi=0$ and $\phi(\underline{r})=0$, that 15 the orlflce Itself. The current I 1 g given by 


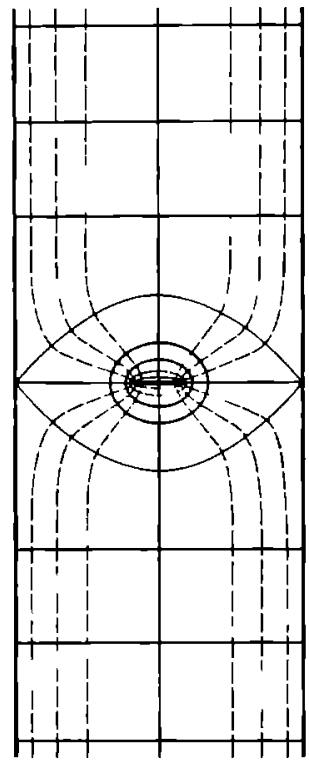

Fig. 1 Point contact in the Maxwell limit $(l<a)$. Indlcated are the electric field lines (broken curves) and the equipotential surfaces.

an integral of the current density $\underline{J}$ over the area $\underline{O}$ of the orifice

$$
I=\int \underline{\mathrm{dO}} \cdot \underline{\mathrm{J}}=\int_{0}^{2 \pi} \mathrm{d} \varphi{\underset{0}{f} \mathrm{a}}_{0} \mathrm{dr} \frac{1}{\rho \mathrm{e}}\left(\frac{\partial \phi}{\partial z}\right)_{\mathrm{z}=0} .
$$

Here, explicit use of Ohm's law is made in the form of $]_{z}=\sigma \mathrm{E}_{z}=$ $=(1 / \rho e)(\partial \phi / \partial z)$, where the conductivity of the metal is given by $\sigma=1 / \rho$. From this, one gets the Maxwellıan resistance for the point contact

$$
R_{M}=\rho / 2 a
$$

Maxwell's method gives the solution for the point-contact problem in the regıme where Ohm's law $\underline{J}=\sigma E$ is valıd. However, for contacts of metals where the mean free path $\ell$ of the electrons is much larger than the linear dimension a of the contact, this assumption does not hold any more. The problem resembles the well known Knudsen-problem in the kınetıc gas theory: By pumping through a small hole in a gas contalner, the pressure of the gas wlll be lowered. However, at the moment where the path length of the gas molecules becomes comparable with the dlameter of the hole, there is no longer diffuse flow and the molecules will pass the orifice ballistıcally. The problem was first considered by Knudsen (1934) and the different regimes are characterızed by the knudsen ratıo $\mathrm{K}=\mathrm{l} / \mathrm{a}$.

Electrical contacts in the clean limit with large Knudsen ratios 


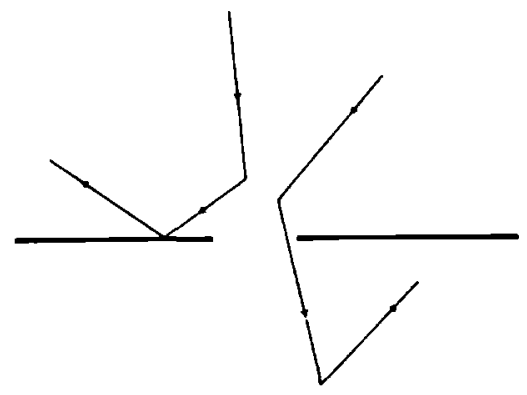

Fig. 2 Point-contact in the Knudsen limit $(\ell>a)$ leading to a ballistic transport of the electrons.

have a large gradient in the potential near the contact, causing the electrons to be accelerated within the metal over a short distance (figure 2 ). Crudely speaking, the electrons are injected with an excess energy from one metal to the other. This type of contacts with large knudsen numbers has been discussed for the first time by Sharvin (1965). The speed increment $\Delta \mathrm{v}$ for an electron which passes the orifice is proportional to the applied voltage: $\Delta v=e V / P_{F}$, where $P_{F}$ is the Fermi momentum. The speed increment results in a current I through the contact, given by $I=\pi a^{2} n \Delta v=$ $\pi a^{2}\left(n_{0} e^{2} / p_{F}\right) v$, where $n_{0}$ is the electron density. Using the Drude formula for the resistivity $\rho=p_{F} / n_{0} e^{2} l$, one finds an expression for the resistance of a contact in the limlt of high $K=l / a: R_{s} \simeq \rho l / \pi a^{2}$. Performing the integration over all possible angles gives a numerical factor of $4 / 3$. The Sharvin resistance $(K=\ell / a \gg 1)$ of a circular contact is therefore given by

$$
R_{S}=\frac{4 \rho \ell}{3 \pi a^{2}} .
$$

It should be realized that the Sharvin resistance is independent of the mean free path $\ell$ because $\rho \alpha 1 / \ell$, as expected for ballistic transport through the contact. Using the expressions for the resistances in the Maxwell and Sharvin limit we can make an estımate of the contact radius a for 1.e. a copper point contact with resistance $\mathrm{R}=1 \Omega$ and residual resistivity ratio between roomtemperature and liquid helium temperature RRR=20. Using the Maxwell formula, we find $a=4 A$, and using the Sharvin formula $a=120 \AA$. For a reasonable pure metal we may conclude that the point contacts usually obtained in these experiments can be consıdered to be in the Knudsen limit $(\ell / a>1)$.

In between the limiting cases of the Maxwell resistance $R_{M}(K \ll 1)$ 
and the Sharvin resistance $R_{s}(K>>1)$, glven by equations (3.4) and (3.5), a simple interpolation formula can be found. Wexler (1966) has given a solution of the Boltzmann equation, using the variational principle for the resistance of a contact. One gets for the resistance $R$ of the point-contact

$$
R=\frac{4}{3 \pi} \frac{\rho \ell}{a^{2}}+\Gamma(K) \frac{\rho}{2 a}=\frac{4}{3 \pi} \frac{\rho \ell}{a^{2}}\left(1+\frac{3 \pi}{B} \Gamma(K) \frac{a}{1}\right) .
$$

Here, $\Gamma$ (K) is a slowly varying function of the Knudsen number $K$, with $\Gamma(\mathrm{K}=0)=1$ and $\Gamma(\mathrm{K}=\infty)=0.694$.

The measured current-voltage characteristic of a $\mathrm{Cu}$ point-contact at low temperatures is non-linear as shown in figure 3. This is clearly visible in the measured first and second derıvatıve (dV/dI and $\mathrm{d}^{2} \mathrm{~V} / \mathrm{dI}^{2}$ ), also plotted in figure 3. The structure in the second derivatıve around 17 and $27 \mathrm{mV}$

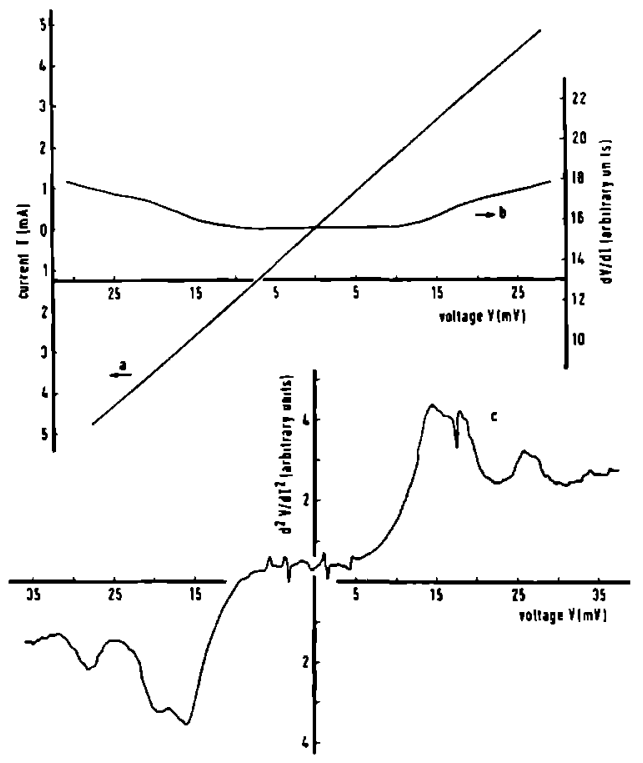

Fig. 3 Recorder output of the current-voltage characterıstics of a copper point contact. The current I (a), the first derıvative $\mathrm{dV} / \mathrm{dI}$ (b) and the second derıvative $\mathrm{d}^{2} \mathrm{~V} / \mathrm{dI}^{2}$ are given as a function of the applied voltage $V$. Resistance $R_{0}=5.7 \Omega$; temperature $\mathrm{T}=1.2 \mathrm{~K}$. 
occurs at energles corresponding to the typlcal phonon frequencies of $\mathrm{Cu}$; therefore the signal is directly related to the electron-phonon interaction in the metal. As the measured signal is proportional to the voltage derivative of the resistance $\left(\mathrm{d}^{2} \mathrm{~V} / \mathrm{dI}^{2}=1 / \mathrm{R} \mathrm{dR} / \mathrm{dV}\right)$, the voltage or energy dependence of the contact resistance as expressed by equation (3.6) has to be consldered. Usually, the electronlc mean free path $\ell$ wlll be energy dependent, $\ell=\ell(E)$. However, in equation (3.6) only the second term depends on the mean free path $\ell(E)$ as the product $\rho \ell$ is characteristic for a glven metal and is independent of the mean free path. One finds for the voltage derivative of the resistance

$$
\frac{d R}{d V}=\Gamma(K) \frac{\rho \ell}{2 a} \frac{d}{d V}\left(\frac{1}{\ell(t=e V)}\right)
$$

The Sharvin-like term in equation (3.6) leads to no structure, but this term is essential as he allows to have an electric field within a metal. The total scattering lengtn $\ell$ of the electrons is determined by the elastic scattering with the impurities ( $\left.\ell_{1 m p}\right)$ and the inelastic scattering with the phonons ( $\ell_{\text {ep }}$ ). Using Matthiessen's rule the total scattering length $\ell$ can be written as

$$
\frac{1}{\ell}=\frac{1}{\ell_{\text {imp }}}+\frac{1}{\ell_{\text {ep }}}
$$

Here, only the electron-phonon length $\ell_{\text {ep }}$ wlll be energy dependent and give rise to a contribution to the signal via equation (3.7). Using the well known Fermi's golden rule argument for the transition rate of an electron arising from the electron-phonon interaction, one finds for the scattering time $\tau_{\text {ep }}(\varepsilon)=\ell_{\text {ep }}(E) / v_{F}$ of an electron with energy $E$ above the Ferml level

$$
\begin{aligned}
\frac{1}{\tau}=\frac{2 \pi}{h} \sum_{k}\left|g_{q}\right|^{2} \delta\left(\varepsilon_{p}-\varepsilon_{k}-\hbar \omega_{q}\right)\left(N_{q}+1-f_{k}\right) \\
+\delta\left(\varepsilon_{p}+h \omega_{q}-\varepsilon_{k}\right)\left(N_{q}+E_{k}\right) .
\end{aligned}
$$

Here, $g_{q}$ is the matrıx element for the electron-phonon interaction for a phonon with wave vector $q$ and energy $h \omega_{q^{\prime}} \varepsilon_{p}$ and $\varepsilon_{k}$ the energies of electrons with momenta $p$ and $k, \mathrm{~N}_{\mathrm{q}}$ the Bose distribution function of the phonons and $f_{k}$ the Fermi distribution function of the electrons. The summation over $k$ can be replaced by an integral 


$$
\Sigma=\frac{\mathrm{N}_{\mathrm{O}}}{2} \rho \mathrm{d} \epsilon_{\mathrm{k}} \rho \frac{\mathrm{gdq}}{2 \mathrm{k}_{\mathrm{F}}^{2}},
$$

where $\mathrm{N}_{0}$ is the density of states at the Fermi level. Defining the Ellashberg function for the electron-phonon interaction as

$$
\alpha^{2} F(\omega)=\frac{N_{o}}{2} \delta \frac{q d q}{2 k_{F}^{2}}\left|g_{q}\right|^{2} \delta\left(h \omega-h_{\omega_{q}}\right),
$$

the scattering time can now be written as

$$
\frac{1}{\tau(\varepsilon)}=2 \pi \int d \omega \alpha^{2} F(\omega)[2 N(\omega)+1+f(\varepsilon+\omega)-f(\varepsilon-\omega)] .
$$

For $\mathrm{T}=0$ this reduces to

$$
\frac{1}{\tau(\varepsilon)}=2 \pi \int_{0}^{\varepsilon / h} d \omega \alpha^{2} F(\omega) \text {. }
$$

Therefore one finds the all important result that for low temperatures the voltage derivative of the point-contact resistance is given by

$$
\frac{\mathrm{dR}}{\mathrm{dV}}(\mathrm{V})=\frac{\rho l}{2 \mathrm{a}} \Gamma(\mathrm{K}) \frac{2 \pi \mathrm{e}}{\hbar \mathrm{v}_{\mathrm{F}}} \alpha^{2} \mathrm{~F}(\mathrm{eV})
$$

This equation allows a direct determination of $\alpha^{2} F$ by measuring the voltage derivative of the resistance of a metallic contact.

In figure 4 we have plotted the measured second derivative $d^{2} v / d I^{2}$ as a function of the applied voltage for a Au point contact. To compare the measured signal with the phonon spectrum, we have given in the same figure the phonon density of states $F(\omega)$ as obtalned from inelastıc neutron scattering experiments. As expected, there is a clear agreement in the voltage dependence of the signal with the frequencies of the transverse and longltudinal phonons. The point-contact spectrum shows a weaker coupling of the electrons with the longltudinal phonons than with the transverse phonons; this was observed for all the noble metal point contacts (Jansen et al., 1977 and 1978). Note, that the point-contact spectrum should vanish for applied voltages bigger than the Debije energy, as there are no more phonons avallable. However, a smooth background sıgnal, saturating at high energles at a non-zero level, remaıns (short-dashed line in figure 4). Because of the spontaneous emission of phonons, the phonon system does not have to be 


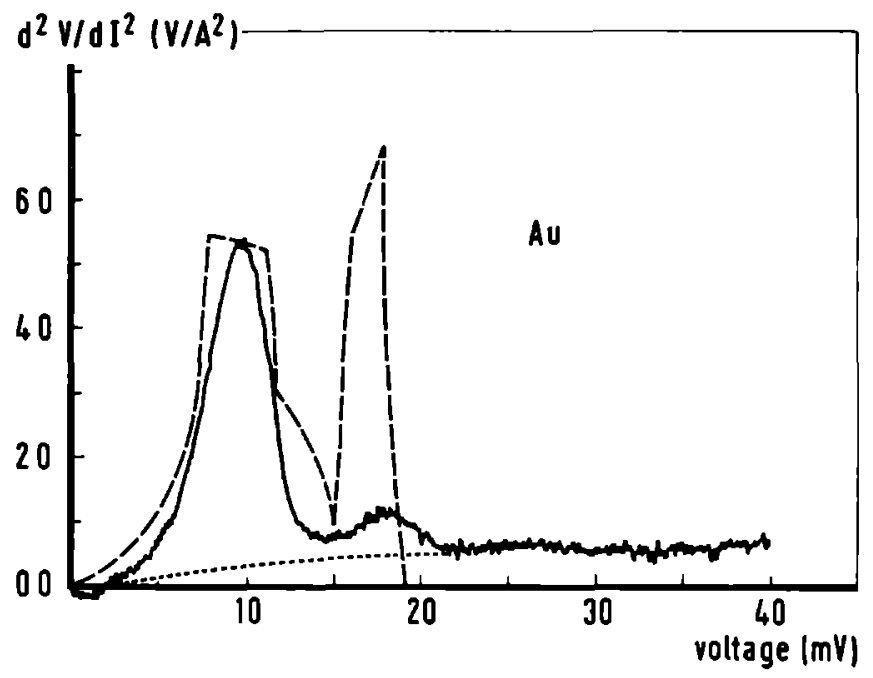

Fıg. 4 Measured $\mathrm{d}^{2} \mathrm{v} / \mathrm{dI}^{2}$ spectrum of a gold point contact. The long-dashed line gives the phonon density of states $F(\omega)$ obtained from inelastic neutron scattering (Lynn et al., 1973). The short-dashed line shows the smooth background signal.

in thermal equilibrium. If this effect is taken into account, it will be shown in the next paragraph, that these non-equilibrium phonons give rise to a background signal, whlch saturates above the Debije energy, in agreement with the experimental findings.

4. Solution of the Boltzmann equation

4.1 Formulation of the problem

In the previous paragraph we have discussed the observed non-linear phenomena in the current-voltage characteristics of metallic point contacts using a more phenomenological approach based on an interpolation procedure between the Maxwell and Sharvin limlt. Here, we present a more fundamental discussion based on the solution of the full non-linear Boltzmann equation for the polnt-contact problem which has recently become avallable (Kulik et al., 1977; Kullk and Yanson, 1978; van Gelder, 1978 and 1980; Jansen et al., 1980 b). The solution is based on an iterative procedure. In zeroth 
order, the linear relation between the current and the voltage is described for a point-contact geometry in the high knudsen number limit, resulting in tile energy-independent Sharvin resistance. In next order, energy-dependent corrections are added to the current leading to the observed nonlinear behaviour of the point contacts. In our discussion, we will generalize the solution of the problem, originally proposed by Kulik et al. (1977), in such a way that the electrons can scatter with arbitrary targets, i.e. phonons, impurities, localized spins, etc. (Jansen et al., 1980 b). To avoid divergent terms in second order, van Gelder (1978 and 1980) has introduced a damping along the trajectory of the electrons. This approach gives in first order (i.e. for a contact with a large knudsen number) the same results as without damping, while in higher orders the iterative procedure converges then apropriately.

First of all the problem has to be formulated in terms of the Boltzmann equation for the distribution function of the electrons, and the Poisson equation for the electric field, taking properly into account the boundary conditions. These equations are then solved by iteration with respect to the collision term in the Boltzmann equation, yielding solutions in zeroth and first order. Because until now point-contact spectroscopy is most successfully used for the experimental determination of the electron-phonon interaction in metals, the result will finely explicitly be applied to phonons, as the scattering targets. In context with the damping mechanism mentioned before, double collision processes will be treated as an application for higher order iterations. It is possible to discuss other interaction mechanisms which can be probed using point contacts within the same theoretical frane-work. In our analysis, we restrict ourselves nainly to a thermalized system of scatterers at a temperature $T=0$, as most of the experiments are performed at liquid helium temperatures. The influence of the temperature, which in essence is only a thermal averaging can easily be taken into account. With respect to the electron-phonon interaction a careful analysis shows that the measured derivative $d^{2} I / d V^{2}$ is proportional to a new electron-phonon interaction-function $\alpha^{2} F_{p}$, where the subscript $p$ indicates the difference with respect to the usual Eliashberg function $\alpha^{2} F$ due to an efficiency factor, which depends on the angle between the momenta of the electron before and after the collision. In the last part of this paragraph the observed smooth background signal on which the $\alpha^{2} \mathrm{~F}$-signal is thought to be superimposed will be discussed. An explanation of the back- 
ground signal is given in terms of a non-equilibrium distribution of the phonons, arlsing from the spontaneous emission-processes in the vicinity of the contact. The analysis yields a saturating signal at high voltages. In addition, phonon-drag effects and direct electron-electron scattering can be assoclated with a structure at zero blas and, respectively, a signal which is linear in the applied voltage.

Formally, to solve the point-contact problem, we have to determine the current I through a polnt contact for an applied voltage $V$. The polnt-contact geometry is represented by an open orıfice in a otherwise, non-conducting plane which separates two metallic half-spaces. The current I is given by an integral of the $z$-component of the current density $J_{z}(\underline{r})$ over the area of the orifice (conveniently placed in the $z=0$ plane):

$$
I=\underset{\text { orifice }}{\int f d x d y ~} J_{z}(x, y, 0)
$$

with the current density per unit volume given by

$$
\underline{I}(\underline{r})=2 \text { e } \sum_{\underline{k}} \underline{v}_{\underline{k}} f_{\underline{k}}(\underline{r})
$$

$\underline{v}_{\underline{k}}$ is the band velocity of an electron with a wave vector $\underline{k}$, and $f_{\underline{k}}$ (r) the distribution function of an electron at the position $\underline{r}$. Once the function $\mathrm{f}_{\underline{k}}(\underline{r})$ is known, the current can ke calculated, and $\underline{f}_{\underline{k}}(\underline{r})$ can be obtained by solving the Boltzmann equation

$$
\underline{v_{k}} \cdot \underline{\nabla}_{\underline{r}} \underline{f}_{\underline{k}}(\underline{r})+\frac{e}{h} \underline{E} \cdot \underline{\nabla}_{\underline{k}} \underline{f}_{\underline{k}}(\underline{r})=\left.\frac{\partial f}{\partial t}\right|_{\text {coll }}
$$

with the drift terms on the left-hand side of this equation and the scattering term on the right-hand side. The electric field E in the Boltzmann equation must be obtalned from Polsson's equation

$$
-\Delta \phi(\underline{r})=e \underline{\nabla} \cdot \underline{E}=-\left(2 e / \varepsilon_{o}\right) \sum_{\underline{k}}^{\sum} f_{k}(\underline{r}),
$$

where $\Delta$ is the Laplacian operator applied to the electrostatic potential energy $\phi(\underline{r})$ and $\varepsilon_{0}$ the dielectric constant of the vacuum. As the ThomasFermi screening length is small (< 1 A) for a metal, the Polsson equation reduces to the condition of charge neutrality

$$
2 e \underset{\underline{k}}{\sum}\left[f_{\underline{k}}(\underline{r})-f_{o}\left(\epsilon_{k}\right)\right]=0
$$

Inside a metal, where $f_{0}\left(\varepsilon_{k}\right)=1 /\left(e^{\left(\epsilon_{k}-\mu\right) / k_{B} T}-1\right)$ is the usual Fermi-Derac distribution function in equilibrium ( $\mu$ is the chemical potential). 
Equations (4.3) and (4.5) have to be solved with the boundary conditions

$$
\begin{aligned}
\phi(z \rightarrow \pm \infty) & = \pm \mathrm{eV} / 2 \\
\mathrm{f}_{\underline{k}}(z \rightarrow \pm \infty) & =\mathrm{f}_{\mathrm{o}}\left(\varepsilon_{\mathrm{k}}\right)
\end{aligned}
$$

becausc a voltage $\mathrm{V}$ is applied over the contact and the distribution has to be in equilibrium and equal to the Fermi function far from the orifice. The collision term for the gain and loss contribution to an electron state with wave vector $\underline{k}$ can be written as

$$
\left.\frac{\partial \underline{f}_{\underline{k}}(\underline{r})}{\partial t}\right|_{\operatorname{coll} 1 .}=\sum_{\underline{k}^{\prime}}\left[\Gamma\left(\underline{k}^{\prime}, \underline{k}\right) \underline{\underline{k}}_{\underline{k}^{\prime}}\left(1-\underline{f}_{\underline{k}}\right)-\Gamma\left(\underline{k}, \underline{k}^{\prime}\right) \underline{f}_{\underline{k}}\left(1-\underline{f}_{\underline{k}}\right)\right] .
$$

According to the general principles of statistical mechanics, the transition rate $\Gamma\left(\underline{k^{\prime}}, \underline{k}\right)$ between an initial state $\mid \underline{k}^{\prime} i>$ and a final state $\mid \underline{k} f>$ is given by

$$
\Gamma^{\prime}\left(\underline{k}^{\prime}, \underline{k}\right)=\frac{2 \pi}{\hbar} \sum_{i f} P_{i} \delta\left(\varepsilon_{f}-\varepsilon_{i}-\left(\varepsilon_{k},-\varepsilon_{k}\right)\right)|<\underline{k} f| u\left|\underline{k}^{\prime} i>\right|^{2} .
$$

$P_{i}$ is the probability for the system to be in the state $i$, and $E_{i}$ and $\varepsilon_{f}$ are the initial and final energies of the target. The squared matrix-element contains the interaction $\mathrm{U}$ between the scattered electron and the target. Arbitrary scattering mechanisms can be chosen for the targets; the most important one is obviously the electron-phonon interaction.

Given the basic formulas for the solution of the problem, the Boltzmann equation (4.3) will be solved, using the charge neutrality condition (4.5) by an iteration with respect to the collision tenn taking into account the boundary conditions (i.e. an electron is specularly or diffusely reflected at the boundaries of the systen). In a schematic way the successive contributions to the current from these iterations are shown in figure 5. Figure 5 a glves the zeroth order field-enission current according to Sharvin's picture (1965). Figures $5 b$ and $5 c$ show the non-linear corrections to the Sharvin current for backflow processes, involving single and double collisions with a target respectively.

\subsection{Solution in zeroth order: Sharvin current}

For the solution of the Boltzmann equation it is convenient to intro- 


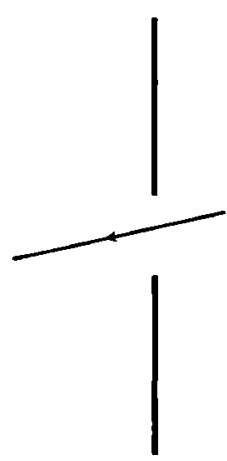

(0)

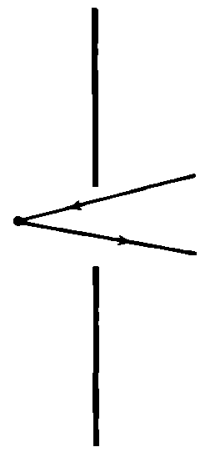

(b)

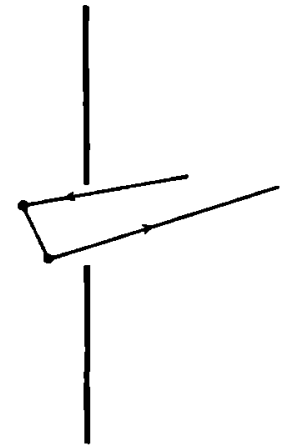

(c)

Fig. 5 Schematic view of the successive iterative contributions to the current through a point contact: zeroth order Sharvin current (a), first order single collision backflow (b) and second order double collision backflow (c).

duce a local potentıal $\psi_{\underline{k}}(\underline{r})$ in the expression for the distribution function $\mathrm{f}_{\underline{k}}(\underline{r})$, defined through

$$
f_{\underline{k}}(\underline{r})=f_{0}\left(\varepsilon_{k}-\psi_{\underline{k}}(\underline{r})\right),
$$

where $f_{0}$ is the Fermi function. With a first order Taylor expansion of the distribution function with respect to this local potential $\dot{\psi}_{\underline{k}}(\underline{r})$ the current density in equation (4.2) becomes

$$
J_{z}(\underline{\underline{r}})=-2 e \sum_{\underline{k}} v_{\underline{k} z} \psi_{\underline{k}}(\underline{r})\left(\frac{\partial F_{o}}{\partial \varepsilon_{k}}\right) .
$$

In the limit of low temperatures this expression reduces to

$$
J_{z}(\underline{r})=2 \mathrm{e}\left\langle\underline{v}_{\underline{k} z} \psi_{\underline{k}}(\underline{r})\right\rangle_{0} \text {, }
$$

where we have used the definıtion $\sum_{k} \ldots \delta\left(\varepsilon_{k}-\omega\right) \equiv\langle\ldots .\rangle_{\omega}$ for a summation over the Fermi-sphere. The distribution function on the left side of the Boltzmann equation (4.3) can also be expanded in a Taylor series, at which procedure only first order terms in the fleld are kept, and one gets 


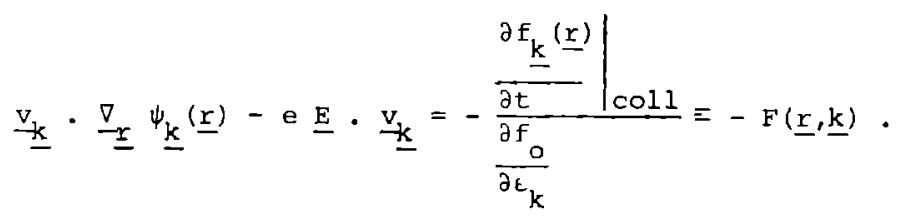

Using Chamber's method of trajectories, we integrate equation (4.12) along the path (varlable $s$ ) of an electron. Introducing $\underline{n}=\underline{\mathbf{v}}_{\underline{\mathbf{k}}} /\left|\mathbf{v}_{\mathbf{k}}\right|$, where $\underline{\mathbf{n}}$ is parallel to the path 5 , equation (4.12) can be written as

$$
\frac{\partial \underline{\underline{k}}}{\partial s}-e \underline{E} \cdot \underline{n}=-\frac{F(\underline{r}, \underline{k})}{\mid v_{k} T}
$$

Integration from $s=-\infty$ (far from the orifice) to $s=0$ (position $\underline{r}$ ) yields an expression for the local potential $\psi_{\underline{k}}(\underline{r})$

$$
\psi_{\underline{k}}(\underline{r})=-[\phi(\underline{r})-\phi(s=-\infty)]-\int_{-\infty}^{0} \frac{d s}{\left|v_{k}\right|} F(\underline{r}, \underline{k}) \text {. }
$$

The potential $\phi(s=-\infty)$ is given by the boundary condition for the starting point of the electron

$$
\phi(s=-\infty)=p\left(\underline{r}, \underline{v_{k}}\right) e v / 2,
$$

where $p\left(\underline{x}, \underline{v}_{k}\right)= \pm 1$, depending on wether the starting point is at the high $(p=+1)$ or low $(p=-1)$ potential side of the contact. The potential $\phi(\underline{r})$ has to be found from the charge neutrality condition (4.5), which can be written as follows

$$
\left\langle\psi_{\mathrm{k}}(\underline{\mathrm{r}})>_{\mathrm{o}}=0\right. \text {. }
$$

Combining equations (4.14) and (4.16), we find for the potential energy

$$
\phi(\underline{r})=\frac{1}{\langle 1\rangle_{0}}\left[\langle\phi(s=-\infty)\rangle_{0}-\langle G(\underline{r}, \underline{k})\rangle_{0}\right] \text {, }
$$

where $G(\underline{r}, \underline{k})=\int_{-\infty}^{0} \frac{d s}{\left|v_{k}\right|} F(\underline{r}, \underline{k})$. It should be noted that $\phi(\underline{r})$ is an even function in the velocity $\underline{-}_{\underline{k}}$ and that therefore the potential $\phi(\underline{r})$ doesn't give a contribution to the current density of equation (4.11). The electrostatic potential $\phi(\underline{r})$ determines the bottom of the conduction band, and plays no direct role in the electronic transport.

Starting with $F=G=0$, it is possible to get an iterative solution 
of the Boltzmann equation, where the collision tern is used to produce the higher order iterations. In zeroth order (1.e. $F=G=0$ ), one finds the fleld-emission current as discussed by Sharvin (1965). The potential energy in this zeroth order 25 then given by

$$
\left.\phi^{(0)}(\underline{r})=\langle\phi(s=-\infty)\rangle_{0} /<1\right\rangle_{0} \text {. }
$$

Introducing $Y(\underline{r})$ as the solid angle at which the orlfice is seen from the position $\underline{r}$, we obtain

$$
\phi^{(0)}(\underline{r})= \pm \frac{\mathrm{eV}}{2}\left[1-\frac{\gamma(\underline{r})}{2 \pi}\right]
$$

with the + or - sign for the two sides of the contact. In particular, we have on the $z$-axis

$$
\phi^{(0)}(x=y=0, z)= \pm \frac{e V}{2} \frac{z}{\sqrt{z^{2}+a^{2}}}
$$

This function has been drawn to indicate the bottom of the conduction band in figure 6. Again in zeroth order, the local potential $\psi_{\underline{k}}^{(0)}(\underline{r})$ is equal to
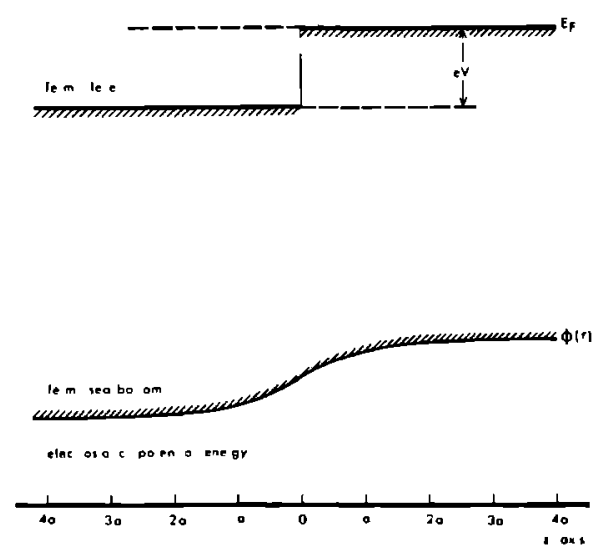

Fig. 6 The conduction band of the electrons for $T \rightarrow 0$ near a contact with radius $a$. The applied voltage over the contact yields the sketched Fermı levels $E_{F}$ and the electrostatic potential energy $\phi(\underline{r})$ for $\underline{r}$ along the z-axis. 


$$
\psi_{\underline{k}}^{(0)}(\underline{r})=-\phi^{(0)}(\underline{r})+\phi(s=-\infty) \text {, }
$$

leading to a zeroth-order distribution function $\mathrm{f}_{\underline{k}}^{(0)}(\underline{r})=f_{0}\left(\epsilon_{\mathbf{k}}-\psi_{\underline{k}}^{(o)}(\underline{r})\right)$ for the elctrons. Thıs $\mathrm{f}_{\underline{k}}^{(0)}(\underline{r})$ is indicated for different positions $\underline{r}$ in figure 7 .

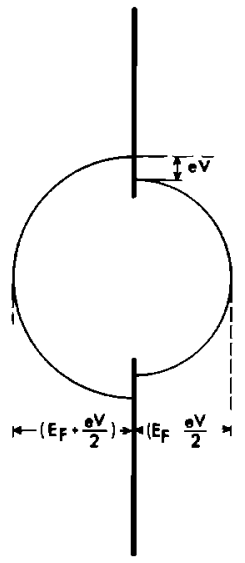

(a)

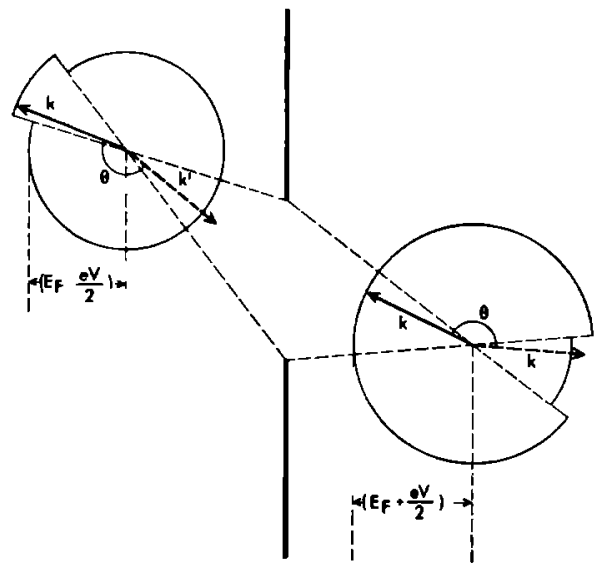

(b)

Fig. 7 The electronic distribution of the electrons in zeroth order according to the Sharvin plcture at the orifice (a) and at positions near the orifice (b). The inelastic scattering processes, where the velocity directions of the electrons change from $\underline{k}$ to $\underline{k}^{\prime}$, give a negative correction to the current.

Using expression, (4..1), the current through the orifice from equations (4.1) and (4.11) becomes

$$
I^{(0)}=e^{2} v D<\left|v_{k z}\right|>_{0} \text {, }
$$

where D is the area of the orifice. Evaluating expression (4.22) for a quadratic dispersion relation for the electrons yields the expression for the Sharvin resistance, already given in the previous chapter for a circular 
orlflce

$$
R_{S}=R_{0}=V / I{ }^{(0)}=\frac{4}{3} \frac{P l}{D} .
$$

$\rho \ell$ is a malerial constant and is given by $\rho \ell=\mathrm{nv}_{\mathrm{F}} / \mathrm{n}_{\mathrm{o}} \mathrm{e}^{2}$ for a Drude model. $R_{S}$ is energy independent and indicates an ohmic behaviour.

In the evaluation of expression (4.22) selt-energy effects have been neglected. If the electronic density of states is not constant within an energy shell eV around the fermi energy, the current ${ }^{(0)}$ through the point contact becomes voltage dependent (non-ohmlc behaviour). Self-energy effects, arısing 1.e. from the electron-phonon interaction, can lead to a non-linear current-voltage characterıstic for a point-contact (Onelyanchuk et al., 1977). By introducing an effectıve mass $m^{*}(E)=m(1+\lambda(c))$, a quadratic dispersion relation for the energy $\varepsilon=h^{2} k^{2} / 2 m^{*}(\varepsilon)$ of an electron can be defined. The renormalisation parameter $\lambda(\varepsilon)$, arising from the electron-phonon interaction, has been discussed in detall by Grimvall (1976); $\lambda$ is connected with the Eliashberg function $\alpha^{2} F$ through $\lambda=\lambda(0)=$ $2 \int \mathrm{d} \omega \alpha^{2} \mathrm{~F}(\omega) / \omega$.

Taking into account this type of self-energy effects, the current in zeroth order takes the form

$$
I^{(0)} \sim \int d k k^{2} v_{k} \sim \int d k k^{2}\left(\frac{d \varepsilon}{d k}\right) \sim \int_{0}^{e V} d \varepsilon \varepsilon_{0} \text {, }
$$

where $\epsilon_{0}=h^{2} k^{2} / 2 m$ is the electron energy without self-energy corrections. Comparing the expressions for $\iota$ and $\varepsilon_{0}$, we have $\left(d \varepsilon_{0} / d \varepsilon\right)=\left(m^{*} / \mathrm{m}\right){ }_{\varepsilon}$. Differentiating expression (4.24) twice gives now the contribution to the second derivative signal due to a change in the electronic density of states because of the electron-phonon interaction:

$$
\frac{1}{R} \frac{d R}{d V} \simeq R_{0} \frac{d^{2} I}{d V^{2}}=\frac{e}{E_{F}} \quad\left(\frac{d \epsilon}{d \epsilon}\right)_{e V}=\frac{e}{E_{F}}\left(\frac{m^{*}}{m}\right)_{e V} .
$$

A quantitative estimate of the non-linearity according to equation (4.25) glves a value $\sim 0.2 \mathrm{v}^{-1}$, which is only a few percent of the usually observed non-linearity in point-contact spectroscopy due to electron-phonon scattering. Therefore, we neglect self-energy effects and assume the density of states of the electrons to be constant near the Fermi energy. 
The first order corrections for the current through a point contact are negative. This is due to the fact that the injected electrons can flow back through the orlfice after an inelastıc scattering with a target. This collision gives rise to an energy-dependent contribution to the current. For 1sotroplc scattering processes, the all important result is found that the change in the resistance of a point contact is proportional to the energy-dependent scattering rate of the electrons.

The first order terms of the electrostatic potential $\phi^{(1)}(\underline{r})$ and the local potentıal $\psi_{\underline{k}}^{(1)}(\underline{r})$ are obtalned by using equations (4.4) and (4.17)

$$
\begin{aligned}
& \Psi_{\underline{k}}^{(1)}(\underline{r})=-\phi^{(1)}(\underline{r})-G_{\underline{k}}^{(0)}(\underline{r}) \\
& \phi^{(1)}(\underline{r})=-\left\langle G_{\underline{k}}^{(0)}(\underline{r})\right\rangle_{0} /\langle 1\rangle_{0} .
\end{aligned}
$$

Here, in the spirit of the Iterative procedure, the function $\mathrm{G}_{\underline{k}}^{(0)}(\underline{r})$ is calculated using the zeroth order distribution function $\mathrm{f}_{\mathrm{k}}^{(0)}$ : $\mathrm{G}_{\underline{k}}^{(0)}(\underline{\underline{r}})=\mathrm{G}\left\{\mathrm{f}_{\mathrm{o}}\left(\varepsilon_{\mathrm{k}}-\psi_{\underline{k}}^{(0)}(\underline{r})\right)\right\}$. Agarn, as $2 \mathrm{n}$ the zeroth order case, $-_{\phi}(1)(\underline{r})$ will not glve a contribution to the current because the potentlal $\phi(\underline{r})$ is an even function of the electron velocity. It turns out that it is not necessary to calculate the electro-static potential explicitly as we could also have defined the local potentıal by $f_{k}(\underline{r})=f_{0}\left(\varepsilon_{k}+\phi(\underline{r})-\psi_{k}(\underline{r})\right)$, with the boundary condition $\psi_{k}(z+\infty)= \pm e v / 2$, in order to get the same results without an explicit expression for $\phi(\underline{r})$. Using equations (4.1) and (4.2), the correction $I^{(1)}$ (backflow current) to the total current is glven by

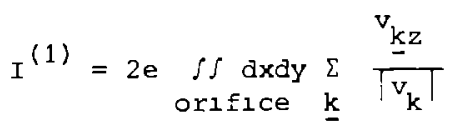

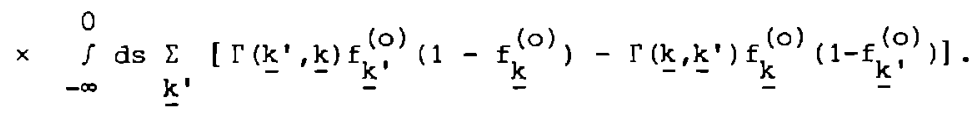

The path s is a straight trajectory parallel to $\underline{\underline{v}}_{\underline{k}}$ ending at the orifice $(s=0)$. In figure 8 we have drawn some illustrative pathes, starting at 


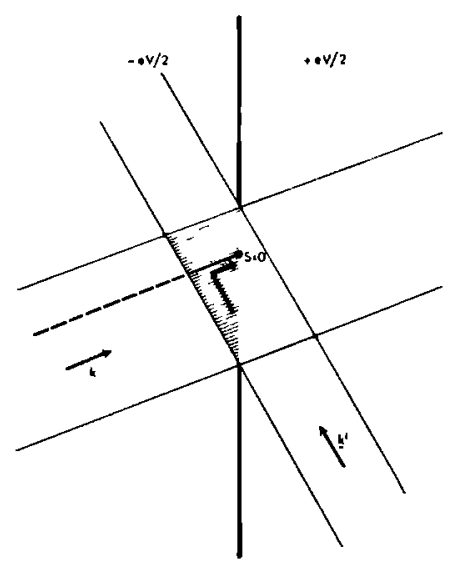

()

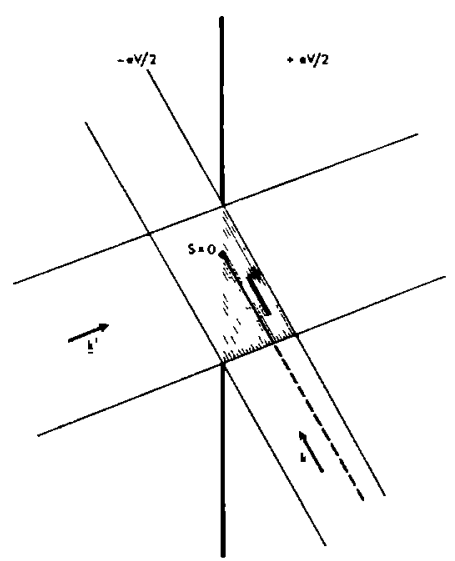

(b)

Fig. 8 Trajectories of the electrons starting far from the left (a) or right (b) side of the orlfice and ending at the orlfice $(s=0)$. The pathlengthes in the shaded area of the figures contribute to the signal for inelastic scattering of the electrons from k' to $\underline{\mathbf{k}}$.

the left side (figure 8a) and at the right side (flgure $8 b$ ) of the orlfice. Only the pathlengthes which lie in the shaded area of the figure can contribute to the current. For both situations, the correction to the current is negative. It is now readily shown that the spatial integrations reduce to a weightfactor $\mathrm{K}\left(\underline{\mathrm{k}}, \underline{\mathrm{k}}^{\prime}\right)=\int \rho \mathrm{dxdy} \frac{\mathrm{v}_{\mathrm{kz}}}{\left|\mathrm{v}_{\mathrm{k}}\right|} \rho$ ds whıch is the common volume of two cylinders constructed with the boundary line of the orifice and whose walls are parallel to $\underline{\mathrm{v}}_{\mathrm{k}}$ and $\underline{\underline{v}}_{\mathbf{k}^{\prime}}$.

In figure 9 we show the iñelastic scattering of an electron in the state $\underline{\mathbf{k}}$ ' to the state $\underline{\mathbf{k}}$ in energy space near the Fermi surface for the temperature $T \rightarrow 0$. In this low temperature limit, the surmation over $k$ and $k^{\prime}$ can be written as

$$
\begin{aligned}
& \text { eV eV eV } \varepsilon_{1}
\end{aligned}
$$

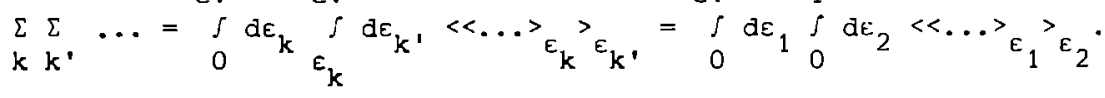




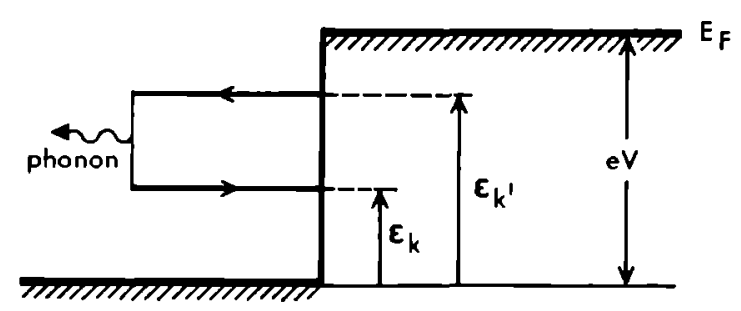

Fig. 9 Single collision backflow process in energy space, where a phonon with energy $\left(\varepsilon_{k^{\prime}}-\varepsilon_{k}\right)$ is emitted spontaneously.

Using these results for the energy and spatial integrations, the backflow current reduces to

$$
I^{(1)}=-2 e \int_{0}^{e V} d \varepsilon_{1} \int_{0}^{\varepsilon_{1}} d \varepsilon_{2}\left\langle\Gamma\left(\underline{k}, \underline{k}^{\prime}\right) K\left(\underline{k}, \underline{k}^{\prime}\right)\right\rangle \epsilon_{1}{ }_{\varepsilon_{2}} .
$$

The influence of the backflow current on the resistance of a point contact can now be evaluated. For small changes in the resistance (small compared with the resistance at zero voltage), the resistance change $\Delta \mathbf{R}$ will be glven by

$$
\Delta R=R_{0}^{2} e \frac{d I^{(1)}}{d(e V)} .
$$

For lsotropıc scattering we obtain

$$
\Delta R=R_{0}^{2} 2 e^{2}\left\langle\left\langle k\left(\underline{k}, \underline{k}^{\prime}\right)\right\rangle_{0}\right\rangle_{0} \int_{0}^{e V} d \varepsilon \Gamma(e V, \varepsilon) N(E) .
$$

Here, $N(E)=\langle 1\rangle_{E}\langle 1\rangle$ o is the normalized density of states. The relaxation time time $\tau$ for an electron with energy $e V$ above the Fermi level is given by

$$
\frac{1}{\tau}(\mathrm{eV})=\sum_{\mathbf{k}^{\prime}} \Gamma\left(\underline{\mathbf{k}}, \underline{\mathbf{k}}^{\prime}\right)=\langle 1\rangle \int_{0}^{\mathrm{eV}} \mathrm{d} \in \Gamma(\mathrm{eV}, \varepsilon) \mathrm{N}(\varepsilon)
$$

whlch also defines the energy dependent relaxation rate $\Gamma(e V, \epsilon)$. Assuming that the density of states is constant for energies $\mathrm{eV} \ll \mathrm{E}_{\mathbf{F}}(\mathrm{N}(\mathrm{eV})=1)$, the resistance change of a point contact at low temperatures is directly 
proportional to the scattering rate

$$
\Delta R=R_{0}^{2} 2 e^{2} \frac{\left.\left.\left\langle\left(\underline{k}, \underline{k}^{\prime}\right)\right\rangle\right\rangle_{0}\right\rangle_{0}}{\langle 1\rangle_{0}} I^{-1}(e v) .
$$

Using $\left.\left\langle\left\langle k\left(\underline{k}, \underline{k}^{\prime}\right)\right\rangle_{0}\right\rangle_{0} /<1\right\rangle_{0}^{2}=a^{3 / 3}$ and $\langle 1\rangle_{0}=m^{2} v_{F} / \pi^{2} h^{3}$, the change in the contact resistance given by expression (4.34) differs only $3.6 \%$ from the expression (3.6) for the resistance found by wexler through an interpolation between the Maxwell and Sharvin limit. The last equation shows the grand result of point-contact spectroscopy. A direct determination of the energy-dependent scattering rate of an electron is possible from the measurement of the resistance of a point contact as a function of the applied voltage. This result can obviously be applied to arbitrary targets (equations $(4.8),(4.32)$ and $(4.34))$.

\subsection{Application to the electron-phonon interaction}

In this section, the theory of point-contact spectroscopy will be explicitly applied to the electron-phonon interaction, starting from the general results of equations (4.28) and (4.30). A transport efficiency function is found for the electron-phonon interaction as measured in pointcontact experiments, very similar to the well-known efficiency function $(1-\cos \theta)$ in the dc-resistivity of bulk metals. Here, we will limit ourself to a thermalized phonon system at a temperature $\mathrm{T}=0$; the consequences of non-equilibrium effects will be treated in the next section. It is necessary to modify the solution of the point-contact problem slightly (van Gelder, 1978 and 1980) by taking into account the damping of the excited electrons in order to deal properly with multiple collision processes.

The collision term for the electron-phonon interaction in the Boltzmann equation can be written as

$$
\begin{aligned}
& \left.\frac{\partial \underline{f_{\underline{k}}(\underline{r})}}{\partial t}\right|_{\text {coll. }}=\frac{2 \pi}{h} \sum_{\underline{k}^{\prime}}\left|g_{k k^{\prime}}\right|^{2}\left[\underline{f}_{\underline{k}},\left(1-\underline{f}_{\underline{k}}\right)\left(N_{q}+1\right)-\underline{f}_{\underline{k}}\left(1-f_{\underline{k}},\right) N_{q}\right\} \delta\left(\varepsilon_{k},-\varepsilon_{k}-h \omega\right) \\
& \left.+\left\{\underline{f}_{\underline{k}^{\prime}}\left(1-f_{\underline{k}}\right) N_{q}-f_{\underline{k}}\left(1-f_{\underline{k}}{ }^{\prime}\right)\left(N_{q}+1\right)\right\} \delta\left(\varepsilon_{k},-\varepsilon_{k}+f \omega\right)\right] .
\end{aligned}
$$


Here, $G_{k k}$, is the matrlx element for the electron-phonon interaction, and $\mathrm{f}_{\mathrm{k}}$ and $\mathrm{N}_{\mathrm{q}}$ the Ferml-Dirac and Bose-Einstein distribution respectıvely, with the phonon wave number given by $q=\left|\underline{k}-\underline{k^{\prime}}\right|$. Using the collision term in the expression (4.28), one gets for tne backflow current in the limit $T=0 \quad\left(N_{q}=0\right)$

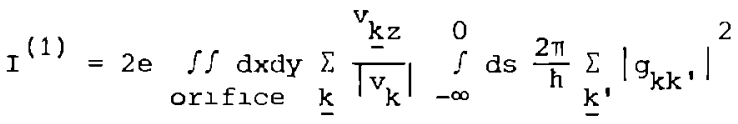

$$
\begin{aligned}
& \times\left[\mathrm{f}_{\underline{k}}^{(0)}\left(1-\mathrm{f}_{\underline{\mathrm{k}}}^{(0)}\right) \delta\left(\varepsilon_{\mathrm{k}},-\varepsilon_{\mathrm{k}}-\hbar \omega\right)-\underline{\mathrm{E}}_{\underline{\mathrm{k}}}^{(0)}\left(1-\mathrm{f}_{\underline{\mathrm{k}}}^{(0)}\right) \delta\left(\varepsilon_{\mathrm{k}^{\prime}},-\varepsilon_{\mathrm{k}}+\hbar \omega\right)\right]
\end{aligned}
$$

With $\underset{\underline{k}}{\sum}=\frac{\mathrm{N}_{\mathrm{o}}}{8 T^{T}} \rho \mathrm{de} f \mathrm{~d}^{2} \mathrm{n}$ (where $\underline{\mathrm{n}}=\underline{\mathrm{v}}_{\underline{\mathrm{k}}} /\left|\mathrm{v}_{\mathrm{k}}\right|$ ) the spatial integrations can be written as

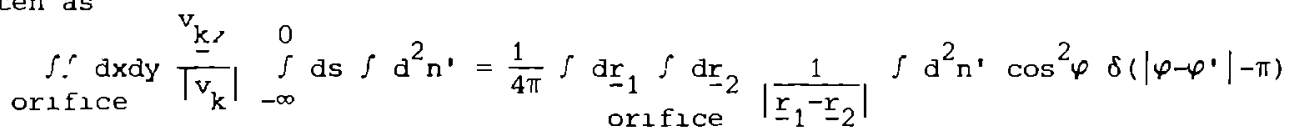

where $\varphi$ and $\varphi^{\prime}$ are the polar angles of $\underline{k}$ and $\underline{k}^{\prime}$, respectively. The coordinates $\underline{r}_{1}$ and $\underline{r}_{2}$ run over the orlfice (see figure 10). Because of the change in the variables of equation (4.37), the transitions during a scatterlng process are no longer restrlcted (l.e. $v_{k z} v_{k^{\prime} z}<0$ ), and therefore the final result has to be multiplied by $\left(\frac{1}{2}\right)$. For the spatial integration one gets

$$
\int d \underline{r}_{1} \int d \underline{r}_{2} \mid \frac{1}{\left|\underline{r}_{1}-\underline{r}_{2}\right|}=\frac{16}{3} \pi \sigma a^{3},
$$

where $\pi \mathrm{a}^{2}$ is equal to the area of the oriflce, and $\sigma=1$ if the shape is circular. Averaging over all possible crystalline directions with respect to the orlfice gives the transport efficiency function $n$, depending on the scattering angle $0=\theta\left(\underline{n}, \underline{n}^{\prime}\right)$ in such a way that $\eta(\theta)=\left\langle\cos ^{2} \varphi \delta\left(\left|\varphi-\varphi^{\prime}\right|-\pi\right)\right\rangle^{*}$. Realizing that $\int d^{2} n \int d^{2} n^{\prime} \sim \int d \alpha \int d \beta \sin \alpha \sin \beta \simeq \pi \rho d 0 \sin \theta(1-\theta / \operatorname{tg} 0)$ $\alpha+\beta=0<\pi$ 0 (see figure 10), one obtains the normalized efficieny function (van Gelder, 1980)

$$
\eta(\theta)=\frac{1}{2}\left(1-\frac{\theta}{\operatorname{tg} \theta}\right) .
$$




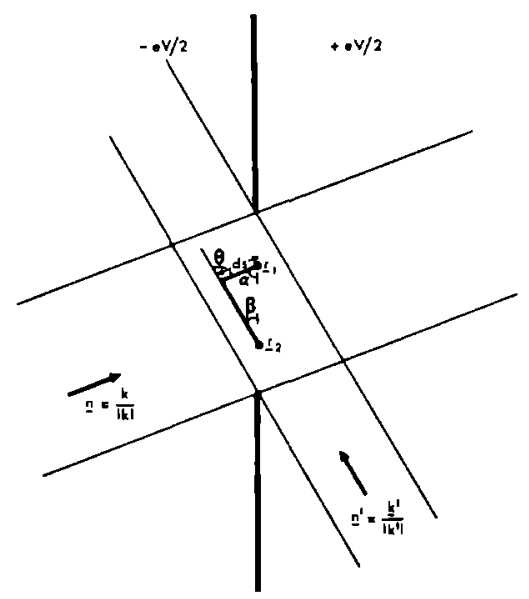

Fig. 10 Integration variables used for the evaluation of the backflow current (see text). If $\underline{n}$ and $\underline{n}$ ' varie in a fixed plane, one has $\int d^{2} n \int d^{2} n \cdot \sim \int d \alpha \int d \beta \sin \alpha \sin \beta \simeq \pi \int d \theta \sin \theta(1-\theta / \operatorname{tg} \theta)$. $\alpha+\beta \quad \theta<\pi$

The backflow current can now be written as

$$
I(1)=\frac{4}{3 \pi} \frac{\mathrm{em}^{2} v_{F}}{h^{4}} a^{3} \int_{0}^{e V} d \varepsilon_{1} \int_{0}^{\varepsilon_{1}} d \varepsilon_{2} \alpha^{2} F_{p}\left(\varepsilon_{2}\right)
$$

where we have used $N_{0}=\langle 1\rangle_{0}=\frac{m^{2} v_{F}}{\pi^{2} h^{3}}$, and the expression for the electronphonon interaction function $\alpha^{2} F_{p}(E)$ for the point contact situation

$$
\alpha^{2} F_{p}(\varepsilon)=\frac{N_{o}}{32 \pi^{2}} \int d^{2} n \int d^{2} n^{\prime}\left|g_{n n^{\prime}}\right|^{2} \eta\left(\theta\left(\underline{n}, n^{\prime}\right)\right) \delta\left(\varepsilon-h \omega_{n n^{\prime}}\right)
$$

In the usual transport theory for the dc-resistivity of bulk metals, one has a simllar expression for the electron-phonon interaction with the well known transport-efficlency function $\left(1-\cos \theta\left(\underline{n}, \underline{n}^{\prime}\right)\right)$

$$
\alpha^{2} F_{t r}(\varepsilon)=\frac{N_{o}}{32 \pi^{2}} \int d^{2} n \int d^{2} n^{\prime}\left|g_{n n^{\prime}}\right|^{2}\left(1-\cos \theta\left(\underline{n}, \underline{n}^{\prime}\right)\right) \delta\left(\varepsilon-\hbar \omega_{n n^{\prime}}\right)
$$

Measurements of the second derivative $d^{2} I / d V^{2}$ for a metallic point contact yield a direct determination of the function $\alpha^{2} F_{p}$ 


$$
\frac{d^{2} I}{d v^{2}}=\left(\frac{4}{3 \pi}\right) \frac{e^{3} m^{2} v_{F}}{h^{4}} a^{3} \alpha^{2} F_{p}(e v)
$$

For the logarithmic derivative of the resistance we obtain for $R \sim R_{0}$

$$
\frac{1}{R} \frac{d R}{d V}=\frac{16}{3} \frac{e}{h v_{F}} \alpha^{2} F_{p}(e V)
$$

It is of some interest to study the qualitative difference between the behaviour of the functions $\alpha^{2} F$ (no efflciency), $\alpha^{2} y_{\text {tr }}$ efficiency $(1-\cos \theta)$ ) and $\alpha^{2} \mathbf{F}_{\mathbf{p}}$ (efficlency $\eta(\theta)$ ) at low frequencies, where the wave vector $q$ of a phonon can be taken as proportional to the frequency $\omega$, for normal and umklapp scatterıng. For the analysıs, we consider a metal with a munimal separation between the spheres in a repeated zone scheme equal to the wave vector $q_{0}$. For the usual normal processes, the different parameters in the function $\alpha^{2} F$ have the following low-frequency dependence: angular integrations $\int d^{2} n \int d^{2} n^{\prime} \sim f q d q \sim \omega^{2}$, squared matrıx element $\left|g_{q}\right|^{2} \sim q \sim \omega$, and the efficiency functions $(1-\cos \theta) \sim \mathrm{q}^{2} \sim \omega^{2}$ and $\eta(\theta) \sim \mathrm{q}^{2} \sim \omega^{2}$. Therefore, In the case of normal processes no difference is expected between $\alpha^{2} F_{\text {tr }}$ and $\alpha^{2} F_{p}$ in the low-frequency behaviour. For umklapp scattering however, the angular integrations yield $\int d^{2} n \int d^{2} n^{\prime} \sim \int\left(q-q_{0}\right) q d q \sim \int q^{2} d q \sim \omega^{3} ; q_{0}$ is the minimal wave vector for the occurrence of an umklapp process. For wave vectors above the minimal value $q_{0}$, the transport efficlencies behave differently: $(1-\cos \theta) \sim$ const., and $\frac{1}{2}(1-\theta / \operatorname{tg} \theta) \sim q^{-\frac{1}{2}} \sim \omega^{-\frac{1}{2}}$. In table I we have summarlzed the obtalned results for the low-frequency behaviour of the functions $\alpha^{2} F, \alpha^{2} F_{t r}$ and $\alpha^{2} F_{P}$.

Table I: Low frequency behaviour of the electron-phonon interaction parameters $\alpha^{2} F, \alpha^{2} F_{t r}$ and $\alpha^{2} F_{p^{\prime}}$ which differ in the transport efficiency, for metals with a spherical Fermi surface.

\begin{tabular}{lccc} 
& $\alpha^{2} F$ & $\alpha^{2} F_{t r}$ & $\alpha^{2} F_{p}$ \\
\cline { 2 - 4 } transport efficiency & - & $(1-\cos \theta)$ & $\frac{1}{2}(1-\theta / \operatorname{tg} \theta)$ \\
normal processes & $\omega^{2}$ & $\omega^{4}$ & $\omega^{4}$ \\
umklapp processes & $\omega^{3}$ & $\omega^{3}$ & $\omega^{5 / 2}$
\end{tabular}


In a careful analysis of the theory of the point-contact problem van Gelder (1978 and 1980) has found that the simple solutions given in Lne preceding section lead to divergencies if the next order approximation, $1 . e$. multiple phonon processes (figure 5c) are considered. It is possiole to overcome these divergence problems by takıng inlo account a damping of the excited electrons along their trajectories. The zeroth order solution of the electron distribution (given in figure 7) is now exponentially damped along the path $s$ of the electron, starting from the orlfice, such that $f^{(0)}=f_{o}\left(\varepsilon_{k}-\psi_{k}(r)\right) e^{-s / l}$, where $s$ is the path length of the electron as measured from the orlfice and $\ell$ the average mean frec path along the trajectory. In zeroth order this damped solution gives exactly the Sharvin current, because the damping is symmetric with respect to the orifice at the $(z=0)$-plane. In first order a damping factor has to be added to the expression for the signal as given by equation (4.44). For point contacts with large Knudsen numbers $(K=l / a>1)$, the damping can be neglected because in the calculations of the backflow current the exponential factor $\mathrm{e}^{-s / \ell}$ is close to one (s $\sim$ a). For double collision backflow processes the solution of the problem is no longer divergent, and van Gelder (1978) has found the relevant geometrical factor for the backflow current to be of the order of $a^{4} \ln (a / \ell)$. The relative strength of the double-phonon signal compared to single-phonon collisions is reduced by a factor $\mathrm{K}=\mathrm{l} / \mathrm{a}$ in the limit of large knudsen numbers $k \gg 1$. In figure 11 we have drawn the double phonon process in energy-space, resulting in a backflow current

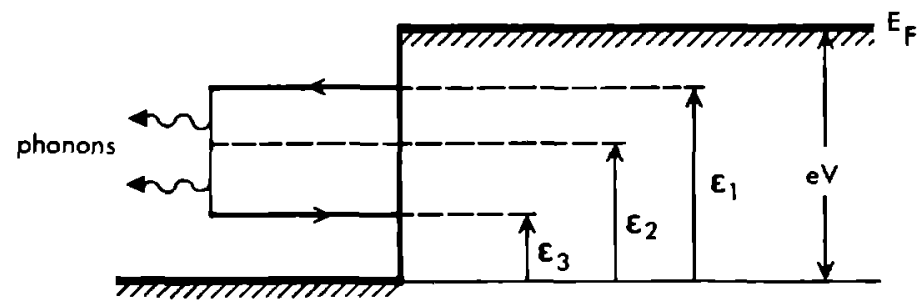

Fig. 11 Double collısıon backflow process in energy space, where two phonons with energy $\left(\varepsilon_{1}-E_{2}\right)$ and $\left(E_{2}-E_{3}\right)$ are emitted spontaneously. 


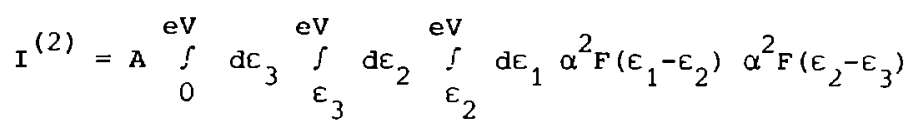

where $A \sim O\left(a^{4} \ln (a / \ell)\right)$ is the geometrical factor. For the second derivative one finds

$$
\frac{d^{2} I(2)}{d V^{2}} \sim \int_{0}^{e V} d E \alpha^{2} F(t) \alpha^{2} F(e V-E)
$$

This result yields a contribution to the signal at twice the relevant phonon frequencies.

\subsection{Background signal}

Although no equilibrium-phonons should be present for energies above the Debije energy $h \omega_{D}$, the measured signal of the point-contact spectrum does not reduce to zero for high applied voltages (eV $\left.>h \omega_{D}\right)$. The signal which can be related to the electron-phonon interaction $\alpha^{2} F_{p}$ and has been discussed in the previous sections, is thought to be superimposed on a smooth background signal. The experiments show that this background signal seems to saturate at a constant level above the Deblje energy. It should be noted that the multiple phonon collisions, discussed in section 4.4 (see equation 4.46), give rise to a signal for energies eV above the Debıje frequency. However, it was shown theoretically and experimentally (van Gelder et al., 1978) that these processes lead to small signals and can certainly not explain the observed background entirely. Very recently (van Gelder et al., 1978 and van Gelder, 1980) a detalled explanation for the background sıgnal was given by taking into account the non-equilibrium distribution of the phonon system. The phonon-emission processes of the injected electrons yield a non-thermalized phonon system near the orlfice leading to additional backflow currents arising from stimulated emission and absorption of phonons. The problem is now to determine the non-thermalized distribution of the phonons which has to be included in the equation (4.40) leading to the signal. One finds as a result a background signal, whlch saturates above the Debije energy. In addition, van Gelder (1980) has also considered processes involving phonon drag effects as a possible explanation of the observed signals at low voltages, and also direct elec- 
tron-electron scattering which gives a signal linear in the applied voltage.

For a determination of the phonon distribution we have to consider the transport equation for the phonons resulting from the electronic distribution given in figure 7 which allows spontaneous emission of phonons. This transport equation is obtained by equalizing the drift and collision term in the Boltzmann equation for the phonons. As usual, the collision term contalns a gain and a loss term; the gain term orlginates from the spontaneous emission of phonons during the decay of the accelerated electrons near the contact, and the loss term describes the production of electron-hole palrs by the phonons. Glving the explicit form of the collision term, the transport equation of the phonons reads

$$
\begin{aligned}
& \underline{\mathrm{c}}_{\underline{\mathrm{q}}} \cdot \frac{\partial \underline{\mathrm{q}}^{(\underline{r})}}{\partial \underline{r}}=\left.\frac{\partial \underline{\underline{\mathrm{q}}}^{(\underline{r})}}{\partial \mathrm{t}}\right|_{\operatorname{coll} 1 .}= \\
& =\frac{\mathrm{C}_{\underline{\mathrm{q}}}}{E \ell_{\mathrm{p}}(\varepsilon)}\left\{\gamma(\underline{r})[1-\gamma(\underline{r})](\mathrm{eV}-E)-\varepsilon \mathrm{N}_{\underline{\mathrm{q}}}(\underline{r})\right\}
\end{aligned}
$$

Here $\underline{q}, \underline{c}_{q}$ and $\varepsilon=h \omega_{q}$ are respectively the wave vector, velocity and energy of a phonon. $l_{p}(\varepsilon)$ is the energy-dependent mean free path of a phonon, and $Y(\underline{r})$ the solid angle under which the orlfice is seen from the point $\underline{r}$. The expression for the collision term can be understood in the following way. The phase-space restrictions with respect to the energy for the gain and loss term, are contained in the second factor between brackets on the right of equation (4.47). For an electron-hole palr creation by a phonon (loss term), the energetic phase-space is equal to the energy $E$ of the phonon, and the rate for these processes is proportional to $E \mathrm{~N}_{\underline{\mathrm{q}}}(\underline{r})$. For a spontaneous emission of a phonon (gain term), the production rate is proportional to the product of the phase-space (eV - E) and the geometrical factor $\gamma(\underline{r})[1-\gamma(\underline{r})]$; the kinematic constraints are contained in the first factor on the right. The loss term is given in the relaxation time approximation by $\mathrm{N}_{\underline{q}}(\underline{r}) / \tau_{p}(E)=\left(c_{\underline{q}} / \varepsilon l_{p}(E)\right) \in N_{\underline{q}}(\underline{r})$.

By introducing the path variable $s$ for the trajectory of a phonon with a wave vector $\mathrm{g}$, the phonon transport equation can be solved and leads to the non-equilibrium phonon distribution function

$$
N_{\underline{q}}(\underline{r})=\frac{e V-\varepsilon}{\varepsilon} \frac{1}{\ell_{p}(\varepsilon)} \int_{-\infty}^{0} d s \gamma(s)[1-\gamma(s)] e^{-s / \ell_{p}(\varepsilon)},
$$


where the path $s$ starts far from the orlfice $(s=-\infty)$ and terminates at the position $\underline{r}(s=0)$ near the orlfice. Assuming that we are in the knudsen regime for the phonon mean free path $\left(K_{p}(c)=\ell_{p}(E) / a \gg 1\right)$ we can ignore the damping term in the integral. For different directions of $q$ the number of phonons present at the orifice $(\underline{r}=0)$ can then be determined to be

$$
\mathrm{N}_{\underline{q}}(0)=\frac{\mathrm{eV}-\varepsilon}{\varepsilon} \frac{1}{l_{\mathrm{p}}(\varepsilon)} \quad \begin{aligned}
& (1 / 4) \text { a for } \underline{\mathrm{q}} \| \text { orıfice } \\
& (\pi / 8) \text { a for } \underline{\mathrm{q}} 1 \text { orıfice. }
\end{aligned}
$$

As an average over all q-directions, we take the numerical factor to be $0.32=1 / 2(1 / 4+T / 8)$ for $\left.\mathrm{N}(c, e V)=\left\langle\mathrm{N}_{\mathrm{g}}(0)\right\rangle\right\rangle_{\mathrm{av}}$. Using this non-equilibrium distribution $\mathrm{N}(t, V)$, we find the contribution to the single collision backflow current by supplementing a factor $(1+N(\varepsilon, v))$ to expression (4.40). In figure 12 we have given the various diagrams in energy space for the different collisions which have to be taken into account. Figures $12 \mathrm{a}$ and $12 \mathrm{~b}$ indicate the spontaneous and stimulated emıssion and the stimulated absorptıon processes, and figures $12 \mathrm{c}$ and $12 \mathrm{~d}$ glve the excitation of an electron by a phonon from below to above the Fermi level. Adding up these contributions, we obtain for the single collision backflow current, with non-equibrium phonons present

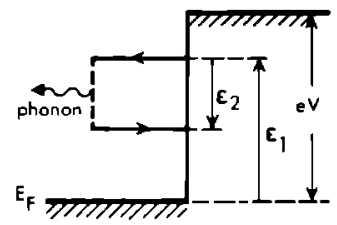

(a)

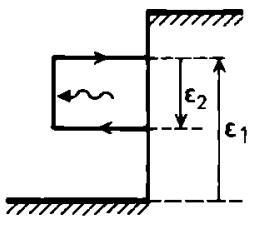

(b)

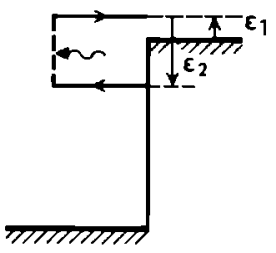

(c)

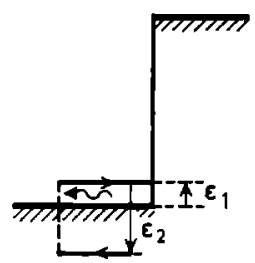

(d)

Fig. 12 Diagrams in energy space for the various contributions to the sıgnal (including background). spontancous and stımulated emission of a phonon (a), stimulated absorption process (b), excitation of an electron from below to above the Ferms level by a phonon (c and $d$ ). 


$$
\begin{aligned}
& I^{(1)}=- \text { const }\left[\int_{0}^{e V} d \varepsilon_{1} \int_{0}^{\varepsilon_{1}} d \varepsilon_{2} \alpha^{2} F_{p}\left(\varepsilon_{2}\right)\left\{1+2 N\left(\varepsilon_{2}, V\right)\right\}+\right. \\
& \left.+2 \int_{0}^{e V} d \varepsilon_{1} \int_{\varepsilon_{1}}^{e V} d \varepsilon_{2} \alpha^{2} F_{p}\left(\varepsilon_{2}\right) N\left(\varepsilon_{2}, v\right)\right]
\end{aligned}
$$

Differentiating twice on gets an expression for the logarithmic voltage derivative of the resistance

$$
\frac{1}{R} \frac{d R}{d V}=\frac{16}{3} \frac{e a}{h v_{F}}\left[\alpha^{2} F_{p}(e V)+\int_{0}^{e V} d \varepsilon \frac{\alpha^{2} F_{p}(E)}{E} G_{1}(E)+\alpha^{2} F_{p}(c) G_{2}(t)\right]
$$

with $G_{1}(E)=1.28 \mathrm{~K}_{\mathrm{p}}^{-1}(\varepsilon)$ and $G_{2}(\varepsilon)=0.64 \mathrm{~K}_{\mathrm{p}}^{-1}(\varepsilon)$. As 1 t can be seen from equation (4.51), the signal $\frac{1}{R} \frac{d R}{d V}$ saturates to a constant level for applied voltages above the Debije energy. Furthermore, the ratio of the background (second and third term) to signal (first term) is of the order $a$ in $\mathrm{R}_{0}^{-\frac{1}{2}}$.

The solution of the Boltzmann equation for phonons leading to equation (4.50) holds for a random generation of phonons with no kinematic constralnts, ı.e. no explicit q-dependence in $\mathrm{N}_{\mathrm{q}}$. If kinematıc constraints are taken into account the expression (4.51) for the signal formally still holds, but the functions $G_{1}(E)$ and $G_{2}(E)$ contaln a welghted q-dependence. An explicit solution of this problem is rather complicated. An excess backfiow of holes would lead to a negatıve signal in the second-derıvative spectra, which is sometımes observed experimentally around zero voltage. In additıon, at low frequencles, where normal scatterıng prevalls, the phonon generation can be peaked in the forward direction, and therefore phonon-drag effects would yield to a backflow of holes through the orıfice, for higher frequencles unklapp scatterıng randomizes the phonon generation and phonon drag is less probable. This, too, would explain the negative signals observed in certain point contacts around zero voltage.

Direct electron-electron interaction, where the collision of two electrons creates an electron-hole, gives a contribution to the signal $\frac{1}{R} \frac{d R}{d V}$ which is linear in the applied voltage as the scattering rate for electron-electron scattering is quadratic in energy. However, if energy and momentum conservation is taken into account this contribution to the signal vanishes. Only if momentum conservation is broken up (for instance by impurities), it is possible to have randon directions of the electrons, and electron-electron collisions could contribute a linear term to the signal. 
5.1 Point-contact Fabrication

Two types of polnt contacts have been investigated in the past, namely shorted-thın-film and pressure-type point contacts. The ploneering first point-contact experıments were performed by Yanson (1974a), using tunneljunction geometries. Usıng conventional evaporatıon technıques, a sandwich of two films of the metal of interest, separated by an oxide layer (figure 13), is produced. Accidentally or intentionally a point contact is produced

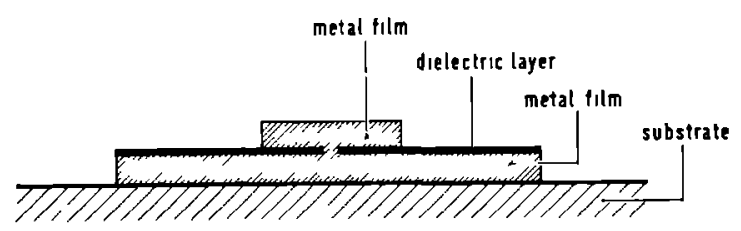

Fig. 13 Schematic view of a metal-insulator-metal tunnel junction with a short circult between the two metal films

In the oxlde layer by an electrıc break-down tnus connecting the two retal fllms by a metallıc short-circult. In later experıments (Yanson and Shalov, 1976), a sharply-pointed steel needle was pressed onto the surface of the junction, providing a crack in the dielectrıc layer and so forming a contact.

Pressure-type contacts are much simpler to control, they have been used for the first time for point-contact spectroscopy by Jansen et al. (1976). A sharply etched metal wire ("spear") is carefully pressed against a flat metal surface ("anvıl") to form the contact. The spear 15 fabricated by electrolytically etching a wire (50-100 $\mu \mathrm{m} \emptyset$ ) to a tip with a curvature radius of $\sim 1 \mu \mathrm{m}$, as is well known from whisker technology (Dozler and Rodgers, 1964). Togetier with the chenically cleaned anvil the spear is then mounted in a system with a differential screw-mechanism which allows to adjust a contact whlle immersed in liquid hellum (see figure 14). The total pltch of the differential screw 1 s of the order of $\sim 20 \mu \mathrm{m}$. In order to increase mechanlcal stabllity, often a spring is bended into the wire forming the spear. 


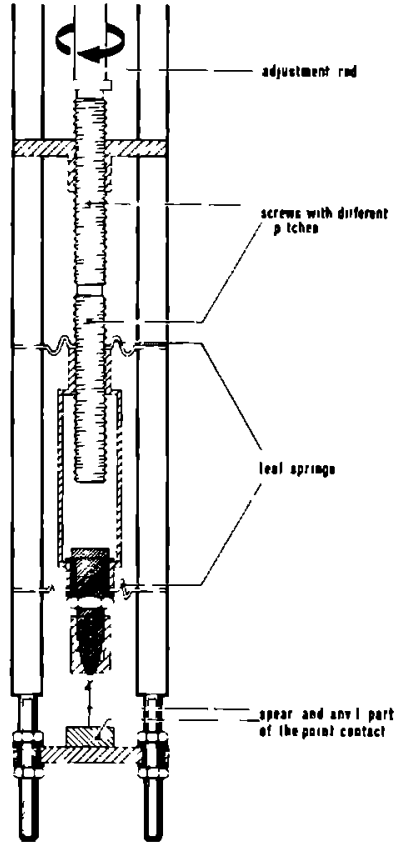

Fig. 14 Schematic view of a

pressure-type point con-

tact. By means of a dif-

ferentıal screw mechanısm

the spear can be moved

towards the anvil in order

to adjust a contact.

Pressure-type point contacts allow the investigation of a large variety of samples, including studies of anısotropies on single crystals. It is possible to obtain stable spear-anvil contacts with resistance values up to $\sim 50 \Omega$. Thin fllm structures have the advantage of higher mechanical stablity with usable contacts of up to $\sim 500 \Omega$. This limit is given by the fact that the contact diameter, calculated with expression (3.5), becomes comparable to the de Broglie wavelength ( $~ 5 \AA)$, where a quantum-mechanical approach of the problem is necessary.

\subsection{Electronics for recording derivatives}

The technique for recording derivatives $\left(\mathrm{dV} / \mathrm{dI}\right.$ and $\left.\mathrm{d}^{2} \mathrm{v} / \mathrm{dI}^{2}\right)$ in pointcontact spectroscopy is the same as in experiments with superconducting tunnel junctions (MCMIllan and Rowell, 1969) which makes use of modulation methods. On top of the bias current, which is swept in order to vary the applied voltage, a small ac-current is superimposed. The ac-voltage over the contact is measured by phase-sensitive detection at the fundamental 
frequency ( $1 \mathrm{f}$-mode) and at twice the fundamental frequency (2f-mode) of the modulator to obtain signals which are proportional to the first and second current-derivatıve of the voltage. As the change $1 \mathrm{n}$ the resistance of the point contacts is usually only a few percent over the whole investigated voltage range, a bridge circult can be used for the ac-signal to compensate the differential resistance in such a way that only changes of the resistance will be seen by the lock-in amplifiers. In figure 15 a typlcal block diagram is glven for the bridge clrcult, with current supply's and

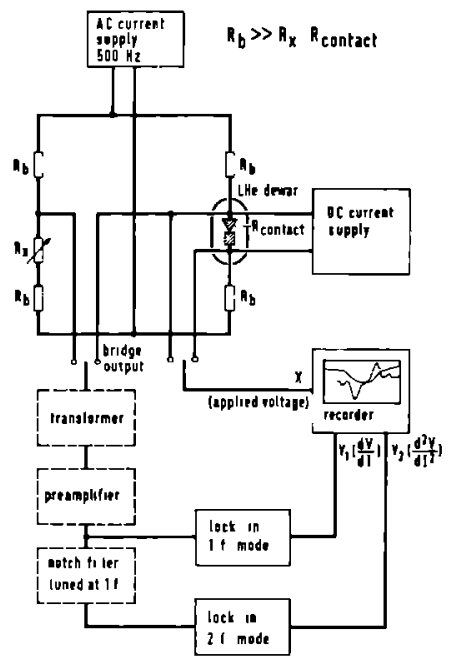

Fig. 15a Block diagram for the phase-sensıtıve detection of first (dV/dI) and second $\left(\mathrm{d}^{2} \mathrm{v} / \mathrm{dI}^{2}\right)$ derivatives as a function of the applied voltage for a point contact at liquid helıum temperatures. In the bridge clrcult the point-contact resistance $R_{\text {contact }}$ is balanced by $R_{x}$. The dashed parts in the block scheme are not essential for the measuring method.

ampliflers, as used in our laboratory (see also Adler and Jackson, 1966). By setting the resistance $R_{x}$ equal to the point-contact resistance, the bridge is balanced for ac-signals. The output of the bridge goes to the two lock-in amplifiers to measure the first and second derivative simultaneously. To avold subharmonic response at the phase-sensitive detector, a 


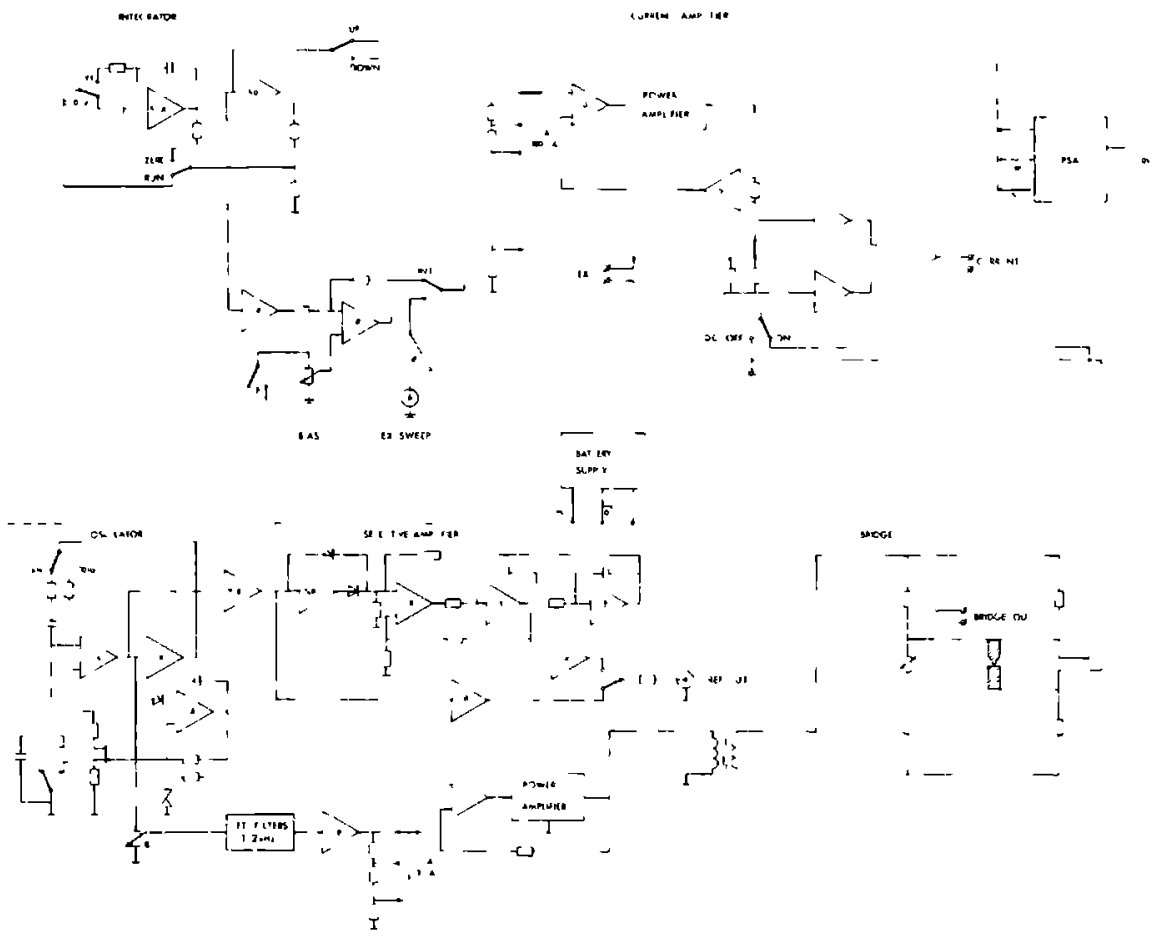

Fig. 15b Detalls of the mann parts of the home-bulld electronic set-up : dc-current supply, ac-current modulator and bridge circuit. The numbers refer to commercially avallable operatıonal amplifiers (Analog Devices and Texas Instruments).

notch filter is used before the $2 f$-mode lock-in. In addition, a transformer can give a better impedance natch to the amplifiers. From the measured contact-resistance $R_{0}$ and the signal $v_{10} 1 \mathrm{n}$ the $1 \mathrm{f}$-mode, both measured at zero bias-voltage, absolute values for the second derlvative $d^{2} v / d I^{2}$ can be obtained from the signal $v_{2}$ in the $2 f$-mode:

$$
\frac{d^{2} v}{d I^{2}}=\frac{4}{\sqrt{2}} v_{2} \frac{R_{o}^{2}}{v_{10}^{2}}
$$

In addition to the intrunsic line width of the spectra, the resolution in the experiment will be determined by the amplitude of the applied ac- 
voltage $\mathrm{V}_{10}$ and the bath temperature $T$. For an ac-voltage with an effective value $v_{10}$, the instrumental 11newıdth is given by $1.72 \mathrm{v}_{10}$ (Kleln et al., 1973). As will be discussed in paragraph 7, the thermal broadening in metalmetal point-contact spectroscopy is the same as in tunneling experiments with normal-metal films where Lambe and Jaklevic (1968) have shown that the thermal innewidth is equal to $5.4 \mathrm{k}_{\mathrm{B}} \mathrm{T}$. Therefore, the temperature of a pumped helium bath $(T=1.5 \mathrm{~K})$ ylelds a linewidth equal to $700 \mu \mathrm{V}$. This value gives an indication for the modulation voltage to be used which is acceptable without averaging the relevant signal instrumentally.

6 Experimental results

In this paragraph we will discuss the experimentally obtalned polntcontact spectra. The first part treats the observed structure in the measured second-derivative spectra whlch, according to the expressions (4.43) and (4.44), can be related directly to the electron-phonon interaction function $\alpha^{2} F_{p}$. The point-contact method has been applied to both superconducting and normal metals. Therefore, it is of considerable interest to compare $\alpha^{2} F_{p^{\prime}}$ as obtained from point-contact spectroscopy while the metal is in the normal state, with $\alpha^{2}$, as obtalned from superconducting tunneling spectroscopy using the Rowell-McMillan inversion scheme. Obviously, pointcontact spectra of normal metals, which do not become superconductors, are particularly important as they give for the first time detalled experimental information about the energy dependent function $\alpha^{2} F$ in normal metals, almost inaccessible otherwise. The experimentally determined point-contact spectra will be compared with reutron-scattering experiments, whlch measure the phonon density of states $F(\omega)$. If theoretical band structure calculation of $\alpha^{2}$ F are avallable, they too can be checked against the point-contact spectra. The all important mass-enhancement parameter $\lambda$, characterizing the electron-phonon interaction for a given metal, can be extracted in a stralght-forward way from the point-contact data and wlll be compared wlth values deduced from other methods or theorles. In the second part of this paragraph, the experimentally observed smooth background signal will be studied in some detall. In particular, a phenomenological analysis to separate the $\alpha^{2}$ F-signal from this background will be glven.

Figure 16 gives an example of a measured spectrum of a $\mathrm{Pb}$ micro-contact in the normal state as performed by Yanson (1974a) in his all important 


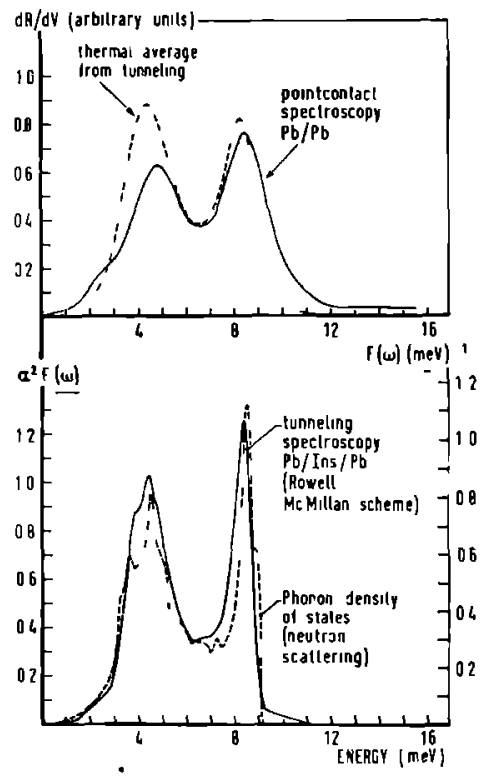

Fig. 16 Measured dR/dV point-contact spectrum of a lead mero-contact in a tunnel Junction (after Yanson, 1974a). Resistance $R_{0}=314 \Omega$; temperature $T=2.0 \mathrm{~K}$. The phonon density of states $F(\omega)$ (Stedman et al., 1967) and the Ellashberg function $\alpha^{2} F(\omega)$ (Rowell et al., 1973) are shown in the bottom part. The dashed line in the top part 15 the thermal average of the function $\alpha^{2} F(\omega)$ due to the bath temperature $\mathbf{T}=2.0 \mathrm{~K}$.

ploneering work, using the shorted-thin-film technique wherc the currentvoltage characteristics of metallic micro-contacts in the oxide layer of a tunnel junction are studied. The usual voltage derıvative of the resistance has been plotted as a function of the voltage applied over the point contact. The transverse and longltudinal phonon peaks are clearly visible at respectively 5 and $8.5 \mathrm{mV}$. In the bottom part of figure 16 we have drawn for a comparison the phonon density of states $F(\omega)$ (Stedman et al., 1967) and the Ellashberg function $\alpha^{2} F(\omega)$ as obtalned from superconducting tunneling spectroscopy (Rowell et al., 1973). Th1s $\alpha^{2}$ F-function has been repeated in the top part of figure 16 again, after averaging with a thermal weight function with line width $5.4 \mathrm{k}_{\mathrm{B}} \mathrm{T}$ (see paragraph 7) in order to make possible a direct comparison with the point-contact spectrum. Note that above the Debije 
energy a small background signal can be observed. The polnt-contact spectrum is very simllar to the smeared Ellashberg function. Small differences are probably due to the transport efficlency function $\eta(\theta)$ in the $\alpha^{2} F_{p}-\operatorname{signal}$ which is most effective at low frequencies. Using short-circuits in tunnel Junctions, the electron-phonon interaction has also been measured in other superconductors, like Sn (Yanson, 1974a) and In (Yanson, 1974b) and has been compared successfully with tunneling spectroscopy.

The use of pressure-type point contacts (Jansen et al., 1976) Increased the applicability of the spectroscopic method to a large variety of samples, including single crystals. In figure 17 we have shown the measured second-derıvatives $\mathrm{d}^{2} \mathrm{~V} / \mathrm{dI}^{2}$ of spear-anvil contacts of the noble metals $\mathrm{Cu}, \mathrm{Ag}$ and $\mathrm{Au}$ (Jansen et al., 1977). In the same figure are also plotted the corresponding phonon densities of states F( $\omega)$ obtalned from inelastic neutron scatterıng (Lynn et al., 1973). Similar results for the noble metals were obtained by electrıc breakdown of the metal-oxide-metal geometry (Yanson and Shalov, 1976; Shalov and Yanson, 1977). All these measured curves show that for the noble metals the electrons are less strongly coupled to the longitudinal phonons than to the transverse phonons. Das (1973) has shown in a theoretical analysis that the d-band character of the noble metals is important for the electron-phonon interaction. Taking into account the hybridization of the $s$ - and d-band electrons the matrix element for the electron-phonon interaction is calculated and it is found that the electrons are strongly coupled to the transverse phonons via umklapp scattering. As is shown by the singularity for $\theta=T$ in the efficiency function $\eta(\theta)$, urklapp scatterıng is partıcularly important in the point-contact spectra leading to $\alpha^{2} F_{P}$. This Idea was confirmed in experiments with real d-metals. In the point-contact spectra of Fe, Co and $\mathrm{N}_{1}$ (Verkın et al., 1979) It can also be seen that the transverse phonons couple strongex with the electrons than the longitudinal phonons do (see paragraph 8). In the case of $\mathrm{Zn}$ and $\mathrm{Cd}$ point contacts, consisting of film structures, however no strong energy dependence was observed in the coupling between the electrons and the phonons (Yanson, 1977).

As there exist detalled calculations for the electron-phonon interaction based on pseudo-potential theory for the alkali metals, polnt-contact spectra of these metals are particularly significant. Experiments of $\mathrm{K}$, Na and $\mathrm{L}_{1}$ have been performed at low temperatures (Jansen et al., 1980a). In figure 18 we have plotted the point-contact spectra for $\mathrm{K}$ and $\mathrm{Na}$, and in figure 19 for 


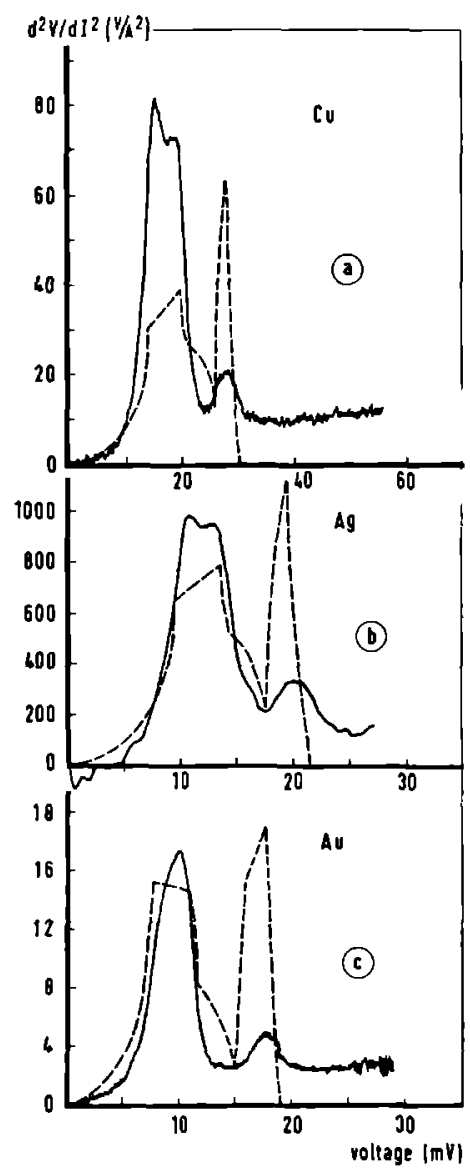

Fig. 17 Measured $\mathrm{d}^{2} \mathrm{v} / \mathrm{dI}^{2}$ spectra of point contacts of the noble metals. Copper contact (a) with resistance $R_{0}=3.3 \Omega$ and at temperature $T=1.5 \mathrm{~K}$; silver contact (b) with $R_{0}=16.3 \Omega$ and at $T=1.2 \mathrm{~K}$; gold contact $(c)$ with $R_{0}=3.3 \Omega$ and at $T=1.2 \mathrm{~K}$. The broken curves give the phonon densities of states $F(\omega)$ obtained from neutron-scattering experiments (Lynn et al., 1973).

Li. In the same figures the phonon densities of states $F(\omega)$, obtained from neutron-scattering experıments are also given. (Cowley et al., 1966; Gilat and Raubenhelmer, 1968; Smlth et al., 1968). The measured spectra for $K$ and $\mathrm{Na}$ look very similar. As expected for metals where normal scattering is important, we observe a stronger coupling for the longltudinal phonons than 

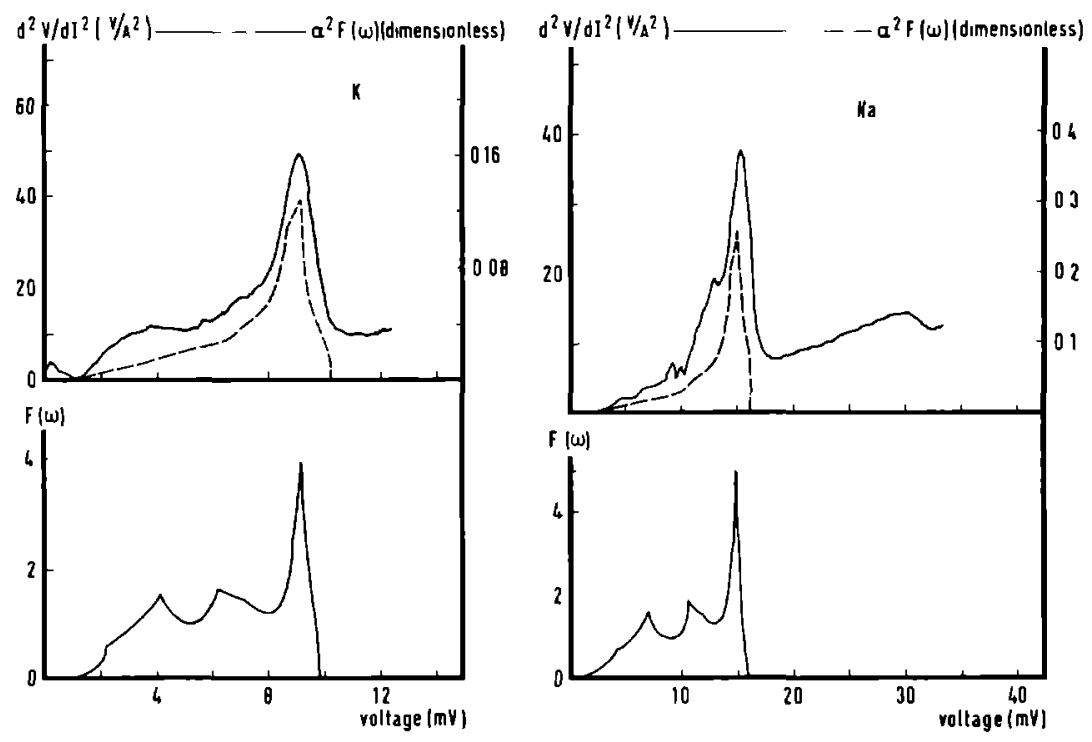

Fig. 18 Measured $\mathrm{d}^{2} \mathrm{v} / \mathrm{dI}^{2}$ spectra for polnt contacts of potassium (left) and sodium (rlght). $\mathrm{K}$ polnt contact with resistance $\mathrm{R}_{\mathrm{O}}=2.9 \Omega$ and at temperature $\mathrm{T}=1.2 \mathrm{~K}$; Na point contact with $\mathrm{R}_{0}=1.1 \Omega$ and at $T=1.5 \mathrm{~K}$. The dashed curves are the theoretically obtalned $\alpha^{2}$ F-functions (Carbotte and Dynes, 1968). The bottom figures give the phonon density of states from neutron-scattering experiments (Cowley et al., 1966; Gllat and Raubenhelmer, 1968).

for the transverse ones. However, for $\mathrm{LI}$ it is agaln seen that the coupling with the transverse phonons is stronger, just as in the case of the noble metals. It has been pointed out in the literature (Danıno et al., 1978) that the Ll metal reveals non-alkali behaviour with much more umklapp scattering than the otner alkalı metals and this could explain the stronger coupling with the transverse phonons. In the figures for the alkali metals, we have also given the theoretically calculated functions $\alpha^{2} F(\omega)$ (Carbotte and Dynes, 1968; Hayman and Carbotte, 1971). The theory is confirmed in a remarkable way by the point-contact experiments. It should be noted that $\mathrm{Na}$ and Ll have a martensitic phase transition (for $\mathrm{Na}$ at $40 \mathrm{~K}$ and for $\mathrm{Ll}$ at $80 \mathrm{~K}$ ) wnlch could give a slight difference in the spectra measured at $1.5 \mathrm{~K}$, 


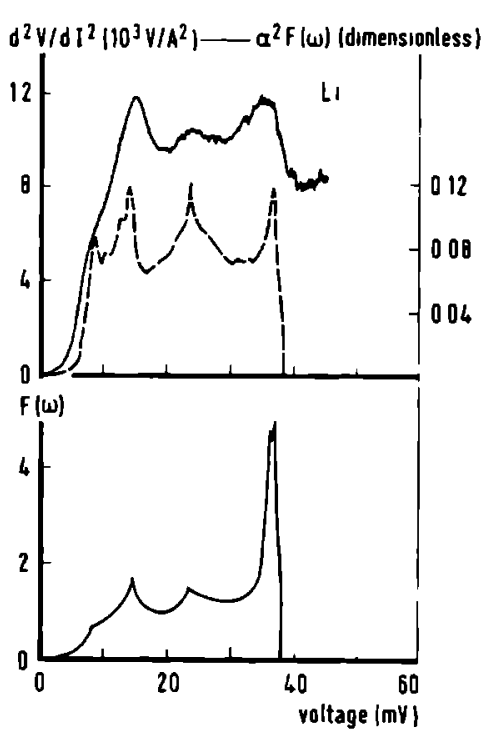

Fig. 19 Measured $\mathrm{d}^{2} \mathrm{v} / \mathrm{dI}^{2}$

spectrum for a lithium

point contact. Resis-

tance $R_{0}=25.4$ and

temperature $\mathbf{T}=1.5 \mathrm{~K}$.

The dashed curve is

the theoretically ob-

talned $\alpha^{2}$ F-function

(Hayman and Carbotte,

1971). The bottom

figure gives the phonon

density of states

from neutron-scatterıng

experiments (Smith et

al., 1968).

compared with the bcc-structure of the alkali metals above the transition temperature used for the theoretical calculations. Note the signal in the spectrum of $\mathrm{Na}$ in figure 18 at twice the phonon frequencies, which is due to double phonon processes as has been observed and explained for Au before (van Gelder et al., 1978). In table II we have summarlzed the point-contact experıments for the metals which have been investigated most extensively for the determination of the electron-phonon interaction. In principle, point-contact spectroscopy can be applied to every metal by means of a short clrcult in a tunneljunction or a pressure contact in a spear-anvil geometry as long as the dimensions of the contact are comparable with the mean free path of the electrons. For instance, in our laboratory we have observed phonon structure in the point-contact spectra of Al, W, Pd, Pt and Mg. By pressing together two different metals, the behaviour of a dissimilar point contact can be investigated. It has been shown experimentally for a Au-Cu junction (Jansen et al., 1977) that both parts of the contact give a contribution to the signal for both negative and positive voltages. As is obvious from figure 8 , where it is lllustrated that scattering processes at both sides of the contact contribute to the current, the addıtıvity of the signals from the two metals is expected. An example of a sandwich junction with a Au-Mg contact is given in figure 20. 
Table II The electron-phonon interaction has been studied in the following metals by means of point contacts (short-circult in a tunnel junction or spear-anvil contact).

\begin{tabular}{|c|c|c|c|}
\hline metal & short-circult & pressure-type & reference \\
\hline $\mathrm{Pb}, \mathrm{Sn}$ & $\mathrm{x}$ & & Yanson, $1974 \mathrm{a}$ \\
\hline In & $x$ & & Yanson, 1974b \\
\hline $\mathrm{Cu}$ & $x$ & & Yanson and Shalov, 1976 \\
\hline $\mathrm{Ag}, \mathrm{Au}$ & $x$ & & Shalov and Yanson, 1977 \\
\hline $\mathrm{Cu}, \mathrm{Ag}, \mathrm{Au}$ & & $x$ & Jansen et al.. 1977 \\
\hline $\mathrm{Zn}, \mathrm{Cd}$ & $\mathbf{x}$ & & Yanson, 1977 \\
\hline $\mathrm{Fe}, \mathrm{Co}, \mathrm{N}_{1}$ & & $\mathrm{x}$ & Verkin et al., 1979 \\
\hline $\mathrm{K}, \mathrm{Na}, \mathrm{Ll}$ & & $x$ & Jansen et al., 1980a \\
\hline
\end{tabular}

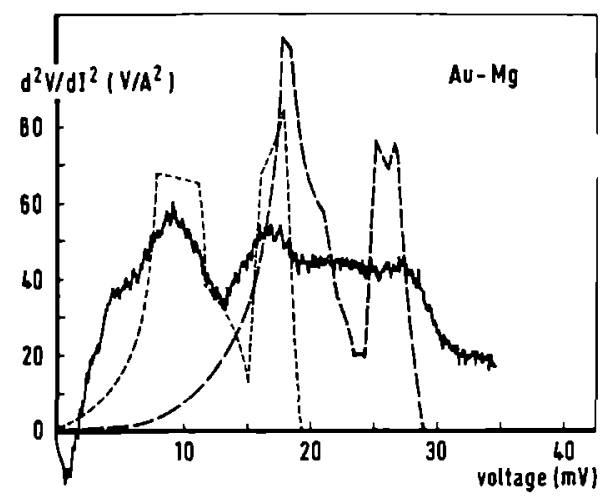

Fig. 20 Measured $d^{2} v / d I^{2}$ spectrum of a dissimilar point contact of a Au spear agalnst a $\mathrm{Mg}$ anvil. Resıstance $\mathrm{R}_{\mathrm{O}}=9.9 \Omega$ and temperature $T=1.5 \mathrm{~K}$. The phonon densitıes of states for Au (short-dashed curve) and for Mg (long-dashed curve) have been obtalned from neutron-scattering experıments (Lynn et al., 1973; Young and Koppel, 1964). 
Pressure-type point contacts allow the investigation of single crystals to look for anisotropy effects. Ooviously, care has to be taken that spear and anvil have the same crystal direction at the point contact. Yanson and Batrak (1978) have measured point-contact spectra for varlous orlentations of a $\mathrm{Zn}$ crystal, which due to its hexagonal structure is expected to show anisotropies. In figure 21 we show the point-contact spectra for two different alrections, where both, the spear and anvil, consisted of properly alıgned single crystals.

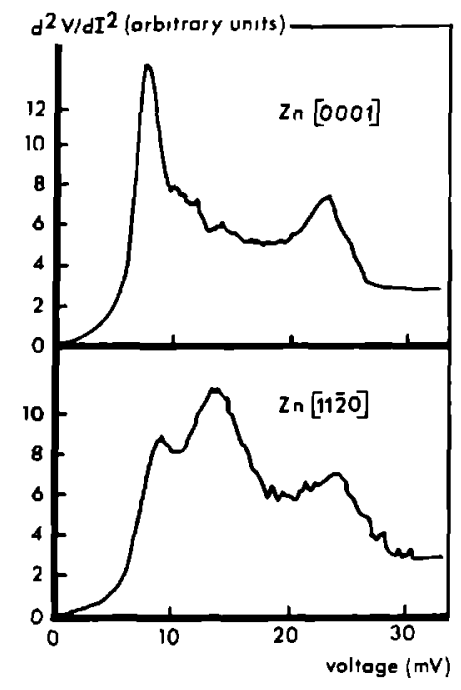

Fig. 21 Measured $d^{2} v / d I^{2}$ spectra for in point contacts along the incilcated crystalline alıections (after Yanson and Batrak, 1978) to show anisotropy effects in point-contact spectroscopy.

The experimental curves show that the phonon structures at 8 and $14 \pi \mathrm{r}$ have a different strength for two perpendicular directions. This observation is in accordance with theoretical calculations of the anisotropy in the function $\alpha^{2} F(\omega)$ for $z$ in (Truant and carbotte, 1973). As expected, for a cubıc metal, such as $\mathrm{Cu}$, the anısotropy effect is very small. It should be noted that the anisotropy is smeared out due to the cosine-dependence of tine injected Sharvin current through the contact $\left(v_{k z}={ }^{\prime} v_{k}\right.$ ' cos $\left.\gamma\right)$.

The preceeding discussion of the results of point-contact experiments has been mainly qualitative in a sense that only the relative energy dependence of the electron-phonon interaction has been discussed, and not its 
absolute strength. However, using the expressions (4.23) and (4.44) and the measured values of the resistance $R_{0}$ and the second derivative $d^{2} v / d I^{2}=$ $R(d R / d V)$ of the contact investigated, the function $\alpha^{2} F_{p}$ can be determined in an absolute way. It is hence possible to get directly the mass-enhancement parameter $\lambda=\int d \omega \alpha^{2} F(\omega) / \omega$, which renormalizes the mass of an electron for the interactions with the phonon system $\left(m^{*}=m(1+\lambda)\right)$, from measured point contact spectra. Van Gelder (1980) has shown that the functions $\alpha^{2} F, \alpha^{2} F_{p}$ and $\alpha^{2} F_{t r}$ yleld the same values for $\lambda$, if the squared matrix element $\left|g_{\Omega}{ }_{n}\right|^{2}$ in the expressions (4.41) and (4.42) can be assured to be proportional to the phonon frequency $\omega$. Because this sum-rule argument for the various $\alpha^{2}$ F-functions with their different transport-efficiencies, it is possible to compare the renormalization parameter $\lambda$, obtained by means of point-contact experiments with values obtalned by other methods. Grimvall (1976) has given a careful literature survey of theoretically and experimentally determined values for the parameter $\lambda$. The theorles involve pseudopotential calculations, and the experimental determinations make use of tunneling spectroscopy and the McMillan equation (relation between $\mathrm{T}_{C}$ and $\lambda$ ) for superconducting materials, or the electrical resistivity and the electronic heat capacity in the limit of high temperatures. In order to calculate the renormalization parameter $\lambda$ the $\alpha^{2} F_{p}$-part in the measured spectra has to be separated from the smooth background signal. In figure 22 point-contact spectra of Au are given for several different contacts. As is clear from the figure, the background signal does not have a uniform voltage dependence for all spectra. The spectra with relatively the lowest background are practically constant above the Debije energy, and therefore are more suitable for a separation in a $\alpha^{2} F_{p}$-signal and a background signal. For this type of the spectra (relatively low background) we assume that the signal $(1 / R) d R / d V$ can be written as a linear combination of two functions $A(V)$ and $B(V)$

$$
\frac{1}{R} \frac{d R}{d V}=p A(V)+q B(V)
$$

where $A(V)$ and $B(V)$ represent the energy-dependent functions for respectively the $\alpha^{2} F_{p}$-signal and the background signal. $p$ and $q$ contaln the energyIndependent factors according to equation (4.47) and contain the linear dimension $a$ of the orifice in such $a$ way that $p \propto a$ and $q \propto a^{2}$. According to the expression for the Sharvin resistance $\left(R_{0} \propto a^{-2}\right)$ we have $P \propto R_{0}^{-\frac{1}{2}}$ and 


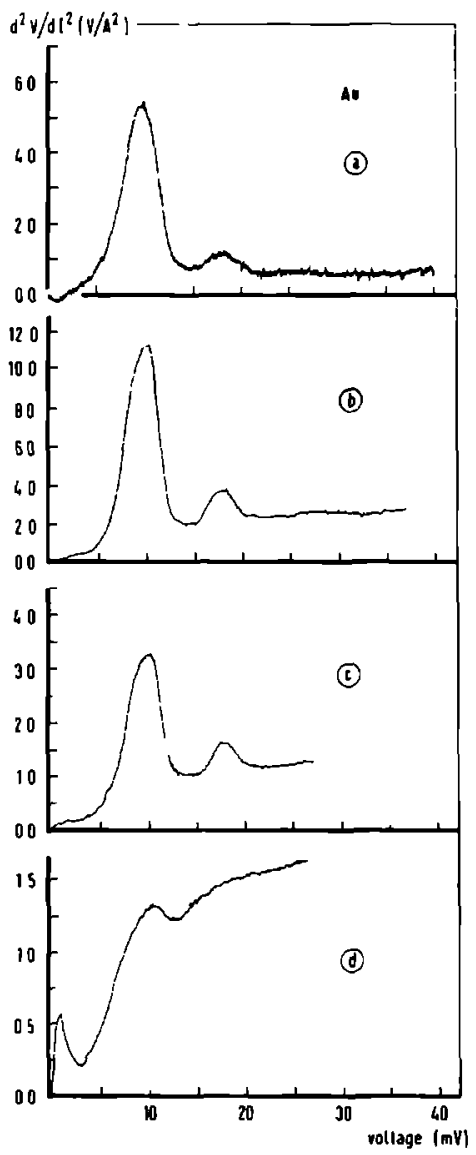

Fig. 22 Measured $d^{2} v / d I^{2}$ spectra for different gold point contacts (pressure type) with resistances $\mathrm{R}_{\mathrm{O}}=2.4 \Omega(\mathrm{a}), 0.7 \Omega(6)$, $0.4 \Omega(c)$ and $0.15 \Omega$ (b), at a temperature $T=1.2 \mathrm{~K}$.

$q \propto \mathrm{R}_{0}^{-1}$. In figure 23 the measured signals at $17.5 \mathrm{mV}$ (transverse phonon peak) and at $35 \mathrm{mV}$ (background) are plotted for different resistances of $\mathrm{Cu}$ point contacts. The data are given on a double log-scale, and we expect respectively a slope of $\left(-\frac{1}{2}\right)$ and of $(-1)$. To be consistent, the signals at the transverse phonon peak have to be corrected for the smooth background signal; using a linear interpolation for the background between low voltages and the Debije voltage, one gets corrected signal values at $17.5 \mathrm{mV}$ (shown as the open circles in figure 23). It is possible to explain deviations from the lines with slope $\left(-\frac{1}{2}\right)$ and $(-1)$ in figure 23 by introducing as additional parameters a transfer probability $t$ and the number $m$ of possible parallel contacts (van Gelder, 1978; van Gelder et al., 1978). The transfer probability 


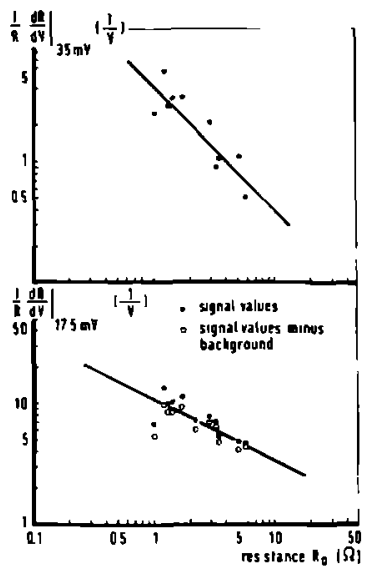

Fig. 23 Measured signal values (1/R) dR/dV for different copper point contacts at applied voltages $17.5 \mathrm{mV}$ (transverse phonon peak) and $35 \mathrm{mV}$ (Deblje energy) as a function of the contact resistance plotted on a double log-scale. From the data at $17.5 \mathrm{mV}$ the background signal has been subtracted (open circles) using a linear interpolation for the background function. According to theory, the straight lines wlth slope $-\frac{1}{2}$ and -1 are expected for respectively the bottom and top figure.

$t$ accounts for oxides and impurities in the contact region which influence the amount of particle transfer through the orifice, while $m$ allows the possibility that a point contact is formed by m parallel current paths. Here, one can assume that the parallel contacts axe roughly equal in size, because for large variations in the contact sizes, only the largest contact would be important. For a transfer probabllity $t$ and a number of contacts $m$ the Sharvin current gets an extra factor $t m$, and the single collision backflow current a factor $t^{2} \mathrm{~m}$ (a factor $t$ for each passage). As the final result we find $p \propto R_{0}^{-\frac{1}{2}} t^{\frac{1}{2}} m^{-\frac{1}{2}}$ and $q \propto R_{0}^{-1} m^{-1}$. Therefore, due to an oxldized or dirty contact $(t<1)$ or due to multiple contacts $(m>1)$, the signal values always decrease. This confirms experimental observations, that the secondderıvatıve sıgnal (related to $\alpha^{2} F_{p}$ ) of great many different point contacts made with one and the same material always seem to have an upper limit which 
obeys the $\mathrm{R}_{0}^{-1 / 2}$-law. The result in figure 23 shows that the spread in the data for the background signal is Jarger than for the $\alpha^{2} F_{p}$-signal. Therefore, it may be possible that the knudsen limut is not fully reached for the phonon mean free path. This conclusion is supported by the fact that often the background still increases above the Deblje energy. Several procedures have been suggested in the literature in order to obtain the $\alpha^{2} F_{p}$-signal from the measured second-derlvative curve with background signal; It has been proposed ad hoc to subtract the background in the form of a hyperbolic tangent (Jansen et al., 1977), to use the measured spectrum that showed no phonon structure (Yanson, 1977) or to interpolate linearly between zero and the Debıje voltage (Jansen et al., 1980a). Takıng into account these considerations, and using the upper limit of the observed signal values in the point-contact spectra, the mass-enhancement parameters $\lambda$ has been estimated for several normal metals. These values for $t$ are glven in table III, together with values from the literature as recomrended by Grimvall (1976). The agreement is rather good. As an amusing side-step,

Table III Renormalization parameters $\lambda=\int_{\int}^{\infty} d \omega \alpha^{2} F(\omega) / \omega$ obtalned from point-contact experıments $\left(\lambda_{p}\right)$ and other methods recommended by Grımvall (1976) $\left(\lambda_{G}\right)$. The critical temperatures $T_{C}$ for superconductivity of the normal metals are predicted by the McMillan equation using the $\lambda_{p}$-values and $\mu^{*}=0.10$.

\begin{tabular}{lccll} 
metal & $\lambda_{p}$ & $\lambda_{G}$ & $T_{C}(K)$ & reference \\
\hline Cu & $0.15 \pm 0.02$ & $0.14 \pm 0.03$ & $410^{-11}$ & see text \\
Ag & 0.15 & $0.10 \pm 0.04$ & $310^{-11}$ & Jansen et al., 1977 \\
Au & 0.16 & $0.14 \pm 0.05$ & $410^{-9}$ & Idem \\
Cd & $0.13 \pm 0.02$ & $0.40 \pm 0.05$ & & Yanson, 1977 \\
$\mathrm{Zn}$ & $0.13 \pm 0.02$ & $0.42 \pm 0.05$ & & Idem \\
$\mathrm{K}$ & $0.13 \pm 0.03$ & $0.13 \pm 0.03$ & $310^{-22}$ & Jansen et al., 1980a \\
$\mathrm{Na}$ & $0.10 \pm 0.03$ & $0.16 \pm 0.04$ & - & Idem \\
$\mathrm{Ll}$ & $0.45 \pm 0.20$ & $0.41 \pm 0.15$ & 2 & Idem
\end{tabular}


we have also given for the normal metals the predicted critical temperature $T_{c}$ for superconductivity, obtained from the MrMillan (1968)-equation

$$
T_{C}=\frac{\theta_{D}}{1.45} \exp \left[\frac{-1.04(1+\lambda)}{\lambda-\mu^{*}-0.627 \mu^{*}}\right],
$$

where $0_{D}$ is the Debije temperature and $\mu^{*} \approx 0.10$ the Coulomb pseudo-potential. The critical temperature for $L 1$ suggests that probably the parameter $\lambda$ snould be smaller. Nevertheless, due to the well known comments on the McMillan equation, these predictions for $T_{C}$ should not be taken too seriously.

In principle it should be possible to scparate the phonon signal from the background, using the reasured spectra of several contacts only and making no use of any interpolation procedure (van Gelder et al., 1980b). We will illustrate this analysis with the spectra for Cu glven in figure 24. All these measured derivatives of the current-voltage characteristic have a background which is nearly constant above the Debije energy. The method makes use of the fact that the function $\alpha^{2} F_{p}$ is equal to zero above the Debije energy. All the spectra are lirst normalızed such that their value at the Deblje energy $\mathrm{eV}_{\mathrm{D}}$ is equal to one. The difference between two normalized spectra will then be proportional to the function $\alpha^{2} F_{p}$ (see figure 25), If It is assumed that tne neasured signal can be considered to be bult up as the sum of two unique functions for all contacts (equation (6.1)). For the 4 spectra in figure 24 we have 6 combinations yielding the function $\alpha^{2} F_{p}$. Each spectrum obtained from a combination is normalized to the same value for the integral $f \mathrm{dV} \alpha^{2} \mathrm{~F}_{\mathrm{p}}(\mathrm{V})$. The average of these difterence functions has now been plotted in figure 25b. The error bars give the rean error of the average, and the first part of the spectrum below $5 \mathrm{mV}$ is shown as a dashed line because of the uncertainty due to the structure at zero blas. From tris analysis one gets for tne ratio of transverse (TA) to longitudinal (LA) phonon peak a value of TA/LA $=6.8$. Using the signal values for the maxumum in a spectrum given in figure 23, we find $\lambda_{\mathrm{Cu}}=0.15 \pm 0.02$. Unfortunately, it is much more difficult to obtain in a simllar way from several measured spectra the functional shape of the backgroand signal. For this purpose, one needs the measured signal $(1 / R) d R / d V$ in absolute units for a determination of the parameters $t$ and $m$ (van Gelder, private communication). Without going into detalls, the results lead to a background function which is very sensitıve to small differences in the two compared spectra, and this rakes it 


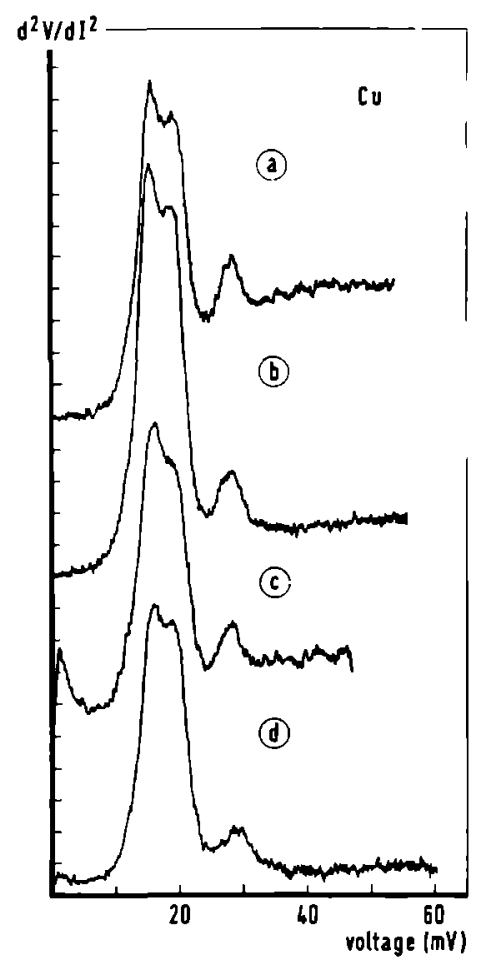

Fıg. 24 Measured $d^{2} v / d I^{2}$ spectra

for different copper point contacts at a temperature $T=1.5 \mathrm{~K}$ with resistances $R_{0}=1.3 \Omega$ (a), $3.3 \Omega$ (b), $5.1 \Omega$ (c) and $5.8 \Omega$ (d) and With signal values 1.9 $\mathrm{VA}^{-2} / \mathrm{dlv}$. (a), $6.3 \mathrm{VA}^{-2} / \mathrm{div}$. (b) $13.1 \mathrm{VA}^{-2} / \mathrm{d} \perp \mathrm{v}$. (c) and $16.9 \mathrm{VA}^{-2} / \mathrm{d} \perp \mathrm{v}$. (d). The spectra are shifted in the vertical scale by 5 scale divisions respectively.

difficult to get a unique determination of the background. Small differences in the measured spectra can very easily be due to different crystaline orlentations near the orlfice.

In some of the experimental curves shown, the spectra often reveal a structure around zero voltage. This kind of structure has been observed on and off in the experiments in a irreproducible way and remains still unexplalned. The $\mathrm{d}^{2} \mathrm{v} / \mathrm{dI} \mathrm{I}^{2}-$ signals can be negatıve or positive at small voltages, corresponding to a maximum or a minimum around zero voltage in the resistance of a contact. In section (4.5) the possibility of zero-bias structures (negative $\mathrm{d}^{2} \mathrm{v} / \mathrm{dI}^{2}-$ signal) due to phonon-drag effects was mentioned. It should be noted that also in the inelastic tunneling spectroscopy zero-bias structure have been observed (Wolf, 1978). Trofimenkoff et al., give an explanation of these effects in terms of "blocking". This means that the transfer of electrons at low voltages is reduced because of ron-equilibrium phenomena, 1.e. the avallable electron states are blocked due to the finite elec- 

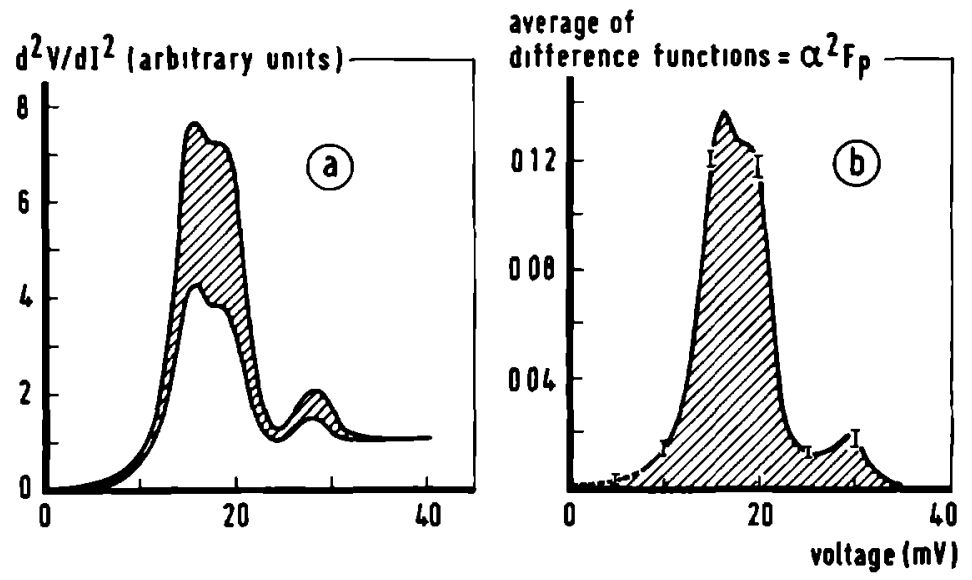

Fig. 25 A typical example to obtain the function $\alpha^{2} F_{p}$ from the difference of two measured spectra normalized to unity at the Debije voltage (35 mV) is given in (a). The average of the 6 difference functions $\alpha^{2} F_{P}$ from the 4 copper point-contact spectra plotted in figure 24 is shown in (b).

tron relaxation rates in the metal at small excitation energies. It is possible that a similar effect could give a maximum in the resistance in point-contact spectroscopy. In additıon magnetıc impurıties are known to cause structure at low voltages in tunnel junctions. Correspondingly, one has been able to show recently with polnt-contact experiments (Jansen et al., 1980b), that magnetıcally dılute alloys give rise to a maximum in the resistance around zero voltage (see paragraph 8). 
7. Temperature dependence of point-contact spectroscopy

In the theoretical analysis of the point-contact problem in paragraph 4 solutions in the low temperature limit have been considered only, as most of the experiments nave been performed at liquid helıum temperatures. In this paragraph, we want to describe the influence of the bath temperature on the experimental results in point-contact experiments (van Gelder et al., 1980 a). For this purpose, just as in the low temperature limit, the collision term in the Boltzmann equation has to be analysed; besides the spontaneous emission processes, the stimulated emission and absorption processes have now to be included as well. As expected, a temperature dependent broadening of the second derlvative spectra is found. In experiments performed between 5 and $300 \mathrm{~K}$ it 15 shown that this broadening is very well described by the theory. In addition, the contact resistance at zero voltage shows a temperature dependence whıch is similar to the temperature dependence of the DC-electrical resistivity of bulk material. However, small differences between the temperature dependence of the bulk resistıvity and that of the point-contact resistance are observed, and it may be possible to explain these effects as being due to the different transport efficiencies for the electron-phonon interaction in the two cases.

The formal solution of the point-contact problem has been given in paragraph 4. Obviously, at temperatures $T \neq 0$, the temperature dependent distribution functions for the phonons and the electrons have to be used explicitly in the zeroth and first order result for the current through a point-contact. In a thermalized system with no non-equilibriur phonons present, this leads to the Bose distribution $v(\omega)$ for the phonons and the Fermi distribution $f_{0}(E)$ for the electrons. In zeroth order, the electronlc distribution is again given by the Sharvin distribution as plotted In figure 7. The zeroth order distribution $f_{\underline{k}}^{(0)}=f_{0}\left(\varepsilon_{k}-e v\right)$ characterızes the electrons which come from the high voltage side of the contact, and the alstribution $\mathrm{f}_{\underline{k}}^{(0)}=f_{o}\left(\varepsilon_{k}\right)$ the electrons coming from the low voltage sıde. Using equations (4.1) and (4.2) the current through a circular contact with radius a is given in zeroth orâer by

$$
I^{(0)}=\frac{3 \pi a^{2}}{4 e \rho l} \int_{-\infty}^{\infty} d \varepsilon\left[f_{0}(\epsilon-c V)-f_{0}(\varepsilon)\right]=\frac{3 \pi a^{2}}{4 \rho l} v \text {. }
$$


The summation over $k$ is performed over half of the Fermi sphere. As can be seen the current $I^{(\bar{o})}$ is independent of the temperature, and in first order no change in the Sharvin resistance $R_{0}=V / I{ }^{(0)}$ is expected. In next order, single collision backflow processes have to be taken into account. In the iterative expression for the backflow current $I^{(1)}$ the full temperature dependent expression for the collision term has to be used (see equations (4.35) and (4.36)). Starting for the iteration again with the zeroth order distribution functions $\mathrm{f}_{\underline{\mathrm{k}}^{\prime}}^{(0)}=\mathrm{f}_{\mathrm{o}}(\varepsilon-\mathrm{eV})$ and $\mathrm{f}_{\underline{\mathrm{k}}}^{(0)}=\mathrm{f}_{\mathrm{o}}\left(\varepsilon_{\mathrm{k}}\right)$ with $\epsilon_{\mathrm{k}}=\varepsilon \pm \hbar \omega$ for the energy of the electron after a spontaneous or stimulated emission $(c-h \omega)$, or a stimulated absorption $(\varepsilon+h \omega)$ of a phonon has taken place, the negative correction $I^{(1)}$ to the current is obtained as

$$
\begin{aligned}
I^{(1)}=-\frac{4 \pi a^{3}}{e v_{F} \rho l} & \int_{-\infty}^{\infty} d \varepsilon\left[f_{0}(\varepsilon-e V)-f_{0}(\varepsilon)\right] \\
& x \int_{0}^{\infty} d \omega \alpha^{2} F_{p}(\hbar \omega)\left[1+2 v(\omega)+f_{o}(\varepsilon+\hbar \omega)-f_{0}(\varepsilon-\hbar \omega)\right] .
\end{aligned}
$$

The factor in front at the right of the last equation is equal to the factor in expression (4.40), If the relations $p l=m v_{F} / n_{0} e^{2}$ and $n_{0}=k_{F}^{3 / 3 \pi^{2}}$ are being used. Note that the correction $I^{(1)}$ to the total current $I$ is of next order in a/ $e_{e p}$ as $I^{(1)} \sim I^{(0)} a / \ell e p$ (see equation (3.12) for the energy dependent relaxation time $\left.\tau(E)=\ell_{\text {ep }}(E) / v_{F}\right)$.

We are interested in the voltage derivatives of the current. For the differential resistance at zero voltage $R_{0}(T)=(d I / d V)^{-1}(V=0)$ we obtain for the case wnere $R_{0}(T)-R_{0} \ll R_{0}$

$$
R_{0}(T)=R_{0}+R_{O}^{2} \frac{4 \pi a^{3}}{v_{F}^{\rho l}} \int_{0}^{\infty} d \omega \alpha^{2} F_{p}(h \omega) \frac{\left(h \omega / 2 k_{B} T\right)}{\sinh ^{2}\left(h \omega / 2 k_{B} T\right)}
$$

Apart from the typlcal difference in the transport efficlency, indlcated by the subscript $p$ in $\alpha^{2} F_{p}(\omega)$, a similar expression is found for the teriperature dependent resistivity $\rho(T)$ in bulk material (Hayman and Carbotte, 1972)

$$
\rho(T)=\frac{2 \pi m}{n_{0} e^{2}} \int_{0}^{\infty} d \omega \frac{\hbar \omega / 2 k_{B} T}{\sinh ^{2}\left(\hbar \omega / 2 k_{B} T\right)} \alpha^{2} F_{t r}(h \omega) .
$$


The full expressions for the electron-phonon interaction function $\alpha^{2} F_{p}$ and $\alpha^{2} F_{t r}$, as weighted with the relevant transport efflclencies, are given by equations (4.41) and (4.42). For the second derivative of the current with respect to the voltage one finds

$$
\frac{d^{2} I}{d V^{2}}=-\frac{4 \pi_{1} a^{3} e}{v_{F} \rho l} \int_{-\infty}^{\infty} d \omega \alpha^{2} F_{p}\left(h_{\omega}\right) \times\left(\frac{h_{\omega-e V}}{k_{B}^{T}}\right)
$$

The function $\chi(L)$ has a bell-shape with half-width $5.4 \mathrm{k}_{\mathrm{B}}^{\mathrm{T}}$.

$$
x(z)=\frac{1}{k_{B} T} e^{z} \frac{(z-2) e^{z}+z+2}{\left(e^{z}-1\right)^{3}}
$$

For $T=0$ expression (7.5) reduces to equation (4.43), and the second derıvative $\mathrm{d}^{2} \mathrm{I} / \mathrm{dV}^{2}$ is directly proportional to the function $\alpha^{2} \mathrm{~F}_{\mathrm{p}}$. Iquation (7.5) describes the broadening of the spectrum arising from a temperature $\mathrm{T} \neq 0$. The same function $x$ for the tremal broadening has been obtalned in an analysis of the temperature dependence of inelastıc tunneling (Lambe and Jaklevic, 1968).

In order to check the predicted broadening experimentally, the second derivative $\mathrm{d}^{2} \mathrm{v} / \mathrm{dI} \mathrm{I}^{2}$ of a point contact has been measured as a function of temperature between helium and room temperature (van Gelder et al., 1980 a). In figure 26 we have given the neasured spectra between 5 and $20 \mathrm{~K}$ for a Au pressure-type point contact showing the expected broadening of the spectrum. As the spectrum will be proportional to $\alpha^{2} F_{P}$ for the lowest measuring temperature, this spectrun can be used to calculate the spectra at higher temperaturcs. By a convolution product with the spectrum at the lowest measuring temperature $\mathrm{T}_{\mathrm{O}}=5 \mathrm{~K}$ we determine the spectra at higher temperatures $\mathrm{T}$ as

$$
\left.\frac{d^{2} V}{d I^{2}}(e V)\right|_{T}=\left.\int_{-10}^{10 k_{B}{ }^{k_{B} T}} d E \frac{d^{2} V}{d I^{2}}(E)\right|_{T_{0}} \times\left(\frac{E-e V}{k_{B}^{T} \text { eff }}\right)
$$

In analogy to the usual quadratic addition of the line widths for the superposition of Gaussian distributions, we have taken an effective temperature $\mathrm{T}_{\text {eff }}=\sqrt{\mathrm{T}^{2}-\mathrm{T}_{\mathrm{o}}^{2}}$ instead of $\mathrm{T}$ in formula (7.7), as we investigate the spectral averaging due to the temperature difference between $T_{0}$ and $T$. The dashed lines in figure 26 give the calculated spectra using equation 


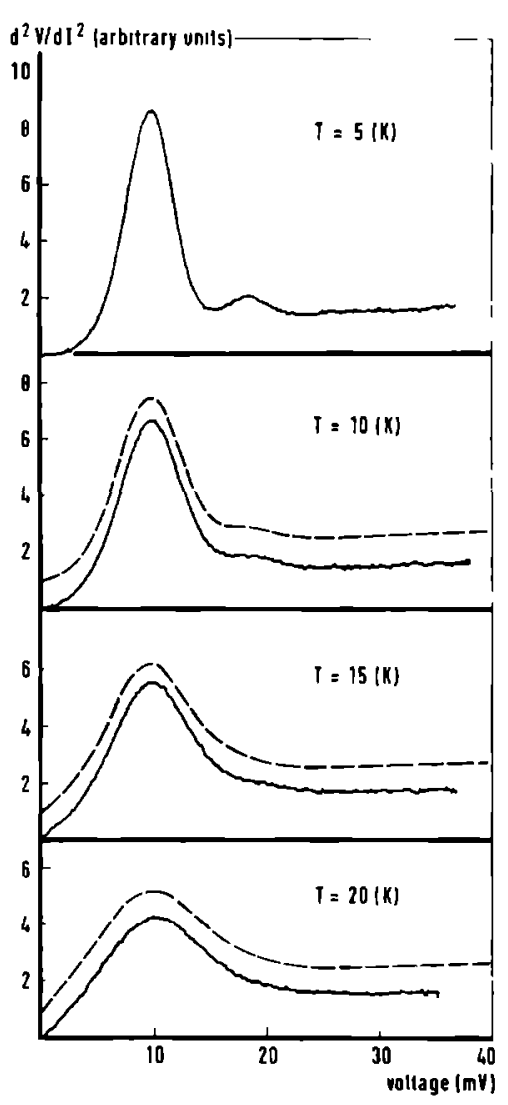

Fig. 26 Measured $d^{2} \mathrm{~V} / \mathrm{dI}^{2}$ spectra at a temporature between 5 and $20 \mathrm{~K}$ for a Au point contact with resistance $R_{0}=5.1 \Omega$. The dashed lines (vertically shifted by one scale division) give the calculated thermal average using the measured spcctrum at $5 \mathrm{~K}$.

(7.7) leading to an almost perfect agreement with the experiments. Due to the use of different kind of materials in the sample holder for the pressure-type point contact, thermal expansion caused instabilities in the Au point contact upon increasing the temperature. To overcome this problem we have investigated $\mathrm{Cu}$ point contacts in a sample holder which was practically totally fabricated from copper. Figure 27 shows a point-contact spectrum between 5 and $270 \mathrm{k}$. Also for this temperature range the thermal broadening fits the theory.

Equations (7.3) and (7.4) show a great similarity between the temperature dependence of the bulk material resistance and that of the point- 


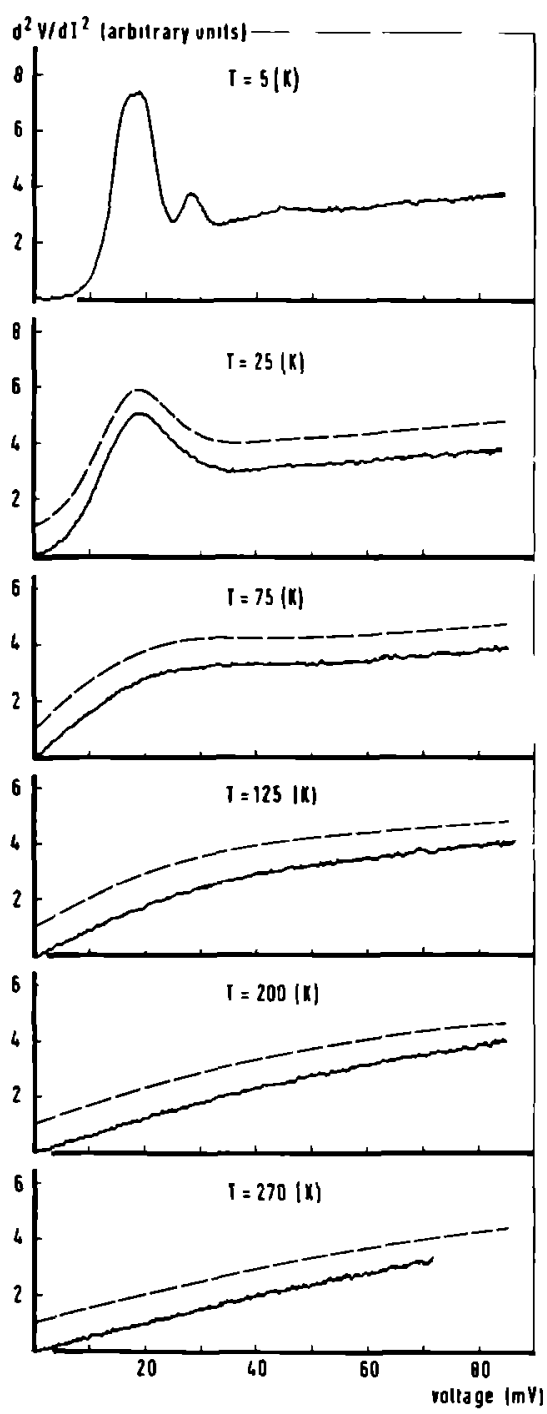

Fig. 27 Measured $d^{2} \mathrm{~V} / \mathrm{dI}^{2}$ spectra at a temperature between 5 and $270 \mathrm{~K}$ for a $\mathrm{Cu}$ point contact with resistance $\mathrm{R}_{\mathrm{O}}=1.7 \Omega$ at $5 \mathrm{~K}$. The dashed lines (vertically shifted one scale division) give the calculated thermal average using the measured spectrum at $5 \mathrm{~K}$.

contact resistance, apart from the typlcal differences in the transport efficiencies. In order to look into this problem experimentally, the pointcontact resistance at zero voltage has been measured for copper as a func- 
tion of the temperature. In figure 28 we have given a neasured contact resistance between 5 and $300 \mathrm{~K}$. In the same figure, also the resistance of a bulk plece of copper wire is shown, using the same material of which the spear-part of the point contact was fabricated. To compare the measured resistances for the two cases, we have plotted them in such a way that they fall together at low temperatures and have the same slope in the limit of high temperatures. The temperature-dependent behaviour for bulk material and a point contact looks similar, rowever there seems to be a characteristic difference in the two experiments. This difference can tentatively be explained in terms of the different transport efficiencics in the functions $\alpha^{2} F_{p}$ and $\alpha^{2} F_{t r}$. This can be analysed in terms of a function $\Delta(T)=$ $T-\left[R_{0}(T)-R_{0}\right] /[d R / d T]$ (see figure 28) whıch corresponds to the difference in the measured resistance and the straight line through $R_{0}(T=0)$ and parallel to the resistance in the high temperature limit. We now look for

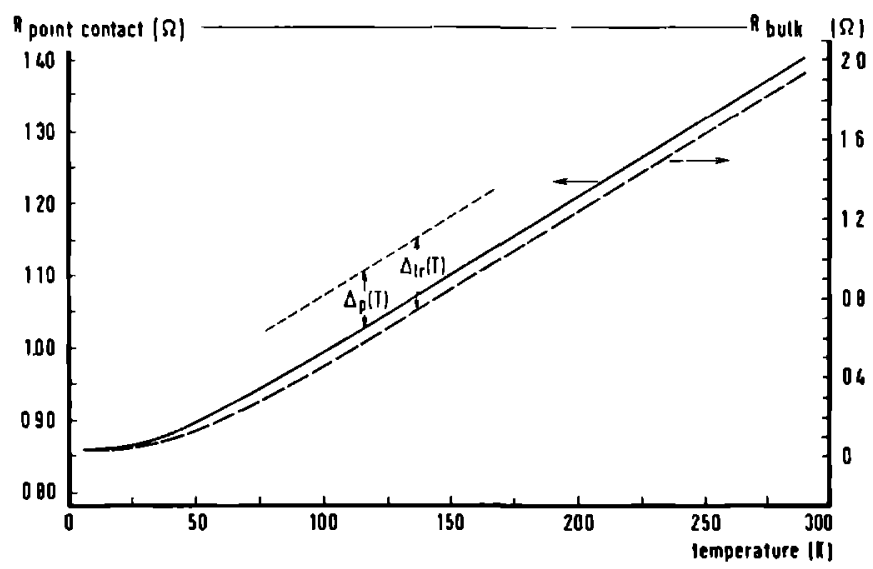

Fig. 28 Resistance at zero voltage of a $\mathrm{Cu}$ point contact as a function of the temperature. The dashed line is the measured resistance of bulk material (spear part of the Cu point contact). The values $\Delta_{p}(T)$ and $\Delta_{t r}(T)$ indicate the difference between the measured resistance values and the stralght line through $R(T=0)$, parallel to the high temperature slope of the resistance.

the difference $\delta\{\Delta(T)\}=\Delta_{p}(T)-\Delta_{t r}(T)$, where $\Delta_{p}(T)$ is related to the point-contact case and $\Delta_{t r}(T)$ to the bulk materlal. It follows from the 
experiments that this difference $\delta\{\Delta(T)\}$ is negatıve. By an evaluation of the functions $\Delta_{p}(T)$ and $\Delta_{t r}(T)$, using the definition and equations (7.3) and (7.4), we find

$$
\Delta(T)=\frac{h}{k_{B}} \frac{0.1 \int_{0}^{\infty} d \omega \alpha^{2} F(\omega)}{2 \int_{0}^{\infty} d \omega \frac{\alpha^{2} F(\omega)}{\omega}},
$$

which holds for temperatures $T$ roughly of the order $T>0.3 \theta_{D}$. Here, we used $\alpha^{2} F_{p}(\omega)$ for a determination of $\Delta_{p}(T)$ and $\alpha^{2} F_{t r}(\omega)$ for $\Delta_{t r}(T)$. Using the sum-rule in the normalizations $\int d \omega \alpha^{2} F_{p}(\omega) / \omega=\int d \omega \alpha^{2} F_{t r}(\omega) / \omega$, we conclude from the experimental found fact $\delta\{\Delta(T)\}<0$ that the function $\alpha^{2} F_{p}(\omega)$ is shifted towards lower frequencies as compared with the function $\alpha^{2} F_{t r}^{P}(\omega)$; this agrees with the $\omega$-dependence of $\alpha^{2} F_{p}$ and $\alpha^{2} F_{t r}$ at low frequencies. In table I of section (4.4) we have shown that for spherical Fermi surfaces unklapp scattering yields an extra factor $\omega^{-\frac{1}{2}}$ for the function $\alpha^{2} F_{p}$ as compared with $\alpha^{2} F_{t r}$. As umklapp scattering is important for a noble metal like $\mathrm{Cu}$, a stronger signal in the point-contact spectrum is expected at low frequencles.

It is clear that further studies are necessary to support these conclusions. For instance, the results in table I are derived for a spherical Ferml surface, and this does not hold in the case of copper. Therefore, it should be interesting to determine in detall the power law of the temperature dependence of the point-contact resistance at low temperatures, in order to investigate the scattering processes in the point-contact geometry as compared with the bulk material.

8. Detection of other scattering mechanisms than the electron-phonon interaction

Most of the experiments in the field of the point-contact spectroscopy carried out until now deal with the measurements of the electronphonon interaction in a metal. It is obviously of great interest whether the polnt-contact method can be applied to study the interaction of the electrons with other elementary excitations in the metal than phonons. The grand result of the solution of the transport problem for the resistance of a point contact is summarized by equation (4.34) which states the all 
important simple fact that the change in the contact resistance as a function of the voltage is proportional to the inverse of the energy-dependent scattering time of an electron. The solution is valid under the condition tnat the inelastic mean free path $\ell(\varepsilon)=v_{F} \tau(\varepsilon)$ is large compared to the linear dimension a of the contact, for this type of junction it is possible to have an electric field within a metal which accelerates the electrons according to the Sharvin plcture (field-emission). In principle, every interaction-process of the conduction electrons in a metal can now be studied with point contacts in the clean limit ( $\ell / a>1)$. In this paragraph we will discuss some recent experiments, which have been performed to investigate the scattering of electrons in a ferromagnet (electronmagnon interaction) and in a magnetically dilute alloy (Kondo-effect). In point-contact experiments on ferromagnetic metals (Verkın et al., 1979), strong anomalies have been observed at voltages which can be correlated with the critical Curie temperature of a ferromagnet. In experiments on Kondo samples (Jansen et al., 1980 b) zero bıas structures have been observed which can be analysed in terms of a direct determination of the relaxation time for the exchange coupling of the conduction electrons with magnetic impurities.

For the study of the electron-magnon scattering Verkin et al. (1979) have investigated point contacts of the ferromagnetic metals $F e$, Co and N1. In the measured second derivative spectra the usual structure was observed at applied voltages which were in agreement with the phonon Erequencies. As an example, we show the spectrum for a NI point contact in figure 29. The observed longltudinal phonon peak is less intense as compared with the transverse phonon peak. Besıdes Fe, Co and N1, the noble metals also revealt this behaviour. This supports the idea of a strong coupling of the d-electrons with the transverse phonons via umklapp scattering in these metals (see paragraph 6). At higher voltages ( $190 \mathrm{mV}$ for $\mathrm{N}_{1}$ ), 1.e. at energles, whlch are of the order of the Curle temperature $\mathrm{T}_{\mathrm{C}}\left(\mathrm{T}_{\mathrm{C}}=627 \mathrm{~K}\right.$ and $\mathrm{k}_{\mathrm{B}} \mathrm{T}_{\mathrm{C}}=54 \mathrm{meV}$ for $\left.\mathrm{N} \mathrm{l}\right)$, a new type of singularity has been observed (see figure 29). This new phenomenon has been explalned by Verkin et al. (1979) by consıdering the strong power dissipation occuring at higher voltages whlch causes local heating of the metal in or near the contact region As the scattering of the electrons in a metal increases at higher temperatures, the mean free path of the electrons gets much smaller and the behaviour of the metallic contacts goes over from the 


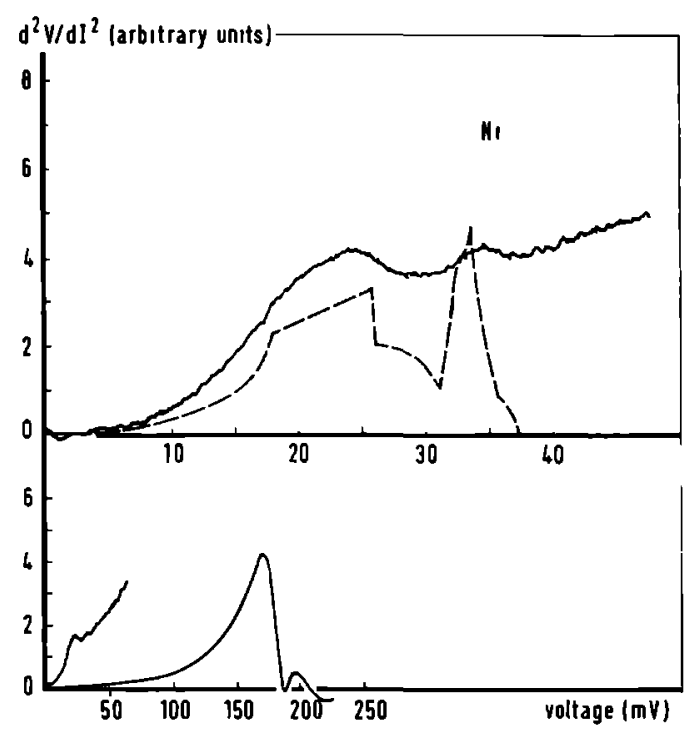

Fig. 29 Measured $\mathrm{d}^{2} \mathrm{v} / \mathrm{dI}^{2}$ spectra for point contacts of $\mathrm{N} \mathbf{1}$ at a temperature $T=1.5 \mathrm{~K}$. Top figure: resistance $\mathrm{R}_{0}=6.5 \Omega$; bottom figure. $R_{0}=2.9 \Omega$. The dashed line gives the phonon density of states obtained from inelastic neutron-scattering experiments (Birgeneau et al., 1964). In the botton figure the singularity around $190 \mathrm{mV}$ is shown together with the expanded signal at low voltages (phonon structure).

Knudsen regime $(l>$ a) into the Maxwell limit $(l<a)$. For reasonably general point-contact geometries of a surounding temperature $T_{b^{\prime}}$ the maximum temperature $\mathrm{T}_{\mathrm{m}}$ at the orifice due to Joule heating is given in the Maxwell 11mit by (Holm, 1967).

$$
\mathrm{T}_{\mathrm{m}}^{2}=\mathrm{T}_{\mathrm{b}}^{2}+\frac{\mathrm{v}^{2}}{4 \mathrm{~L}}
$$

This simple relation has been deduced by assuming that the electronic heat conductivity $K$ and the electrical resistıvity $\rho$ are related by the Wiedemann-Franz law $K \rho=T L$ ( $L$ Is the Lorentz number). For $T_{b} \rightarrow 0$, the maximum temperature $\mathrm{T}_{\mathrm{m}}$ is proportional to the applied voltage $\mathrm{V}$, and equation ( 8.1 ) gives numerically for the second term $(3.2 \mathrm{v})^{2}$ if $\mathrm{v}$ is measured in millivolts. The fact that conslderable heating in the vicinity 
of the metallic contact takes place is supported by the occurrence of an irreversible lowering of the point-contact resistance at high voltages ( $200 \mathrm{mV}$ ) which is probably due to the disapprarance of strain hardenirg In the metal of the pressure-type point contact at a certain temporature, called the softening temperature $T_{\text {soft }}$ (for $\mathrm{N}_{1}, \mathrm{~T}_{\text {soft }}=520 \mathrm{~K}$ ). For voltages where $T_{m} \sim T_{c}$, the resistance of the contact $(R=p / 2 a)$ changes abruptly due to the increase of the electron-magnon resistivity at the Curle temperature. Assuming that the change in temperature spreads out spherically in the vicinity of the contact, the experimental results can be simulated using a step-like model for the temperature dependence of the magnon resistivity (Verkin et al., 1979). This model predicts a singularity at the voltage $v_{C}=3.6 T_{C}$ which is in agrecment with the experiment. As a conclusion, one is tempted to say that in these pointcontact experiments only the temperature dependence of the bulk resistivity, arısing from electron-magnon scatterıng, is determined. Here, the pointcontact method is not used in the sense of a tool to measure the energy dependence of the scattering time for the electron-magnon interaction.

In an other series of polnt-contact exporiments, where Mo has been pressed against the magnetically ordering metals $\mathrm{GdCu}_{2} \mathrm{Sl}_{2}$ and $\mathrm{Tb}$ (Leppin and wohlleben, 1978), structure has been observed and ascribed to electronmagnon scattering. As the $\mathrm{GdCu}_{2} \mathrm{Sl}_{2}$ sample is certainly not pure, the contacts are probably again in the Maxwell limit and local heating effects can explain the observed increase in the resistance, just as in the case of $\mathrm{Fe}$, Co and $\mathrm{N} \mathbf{l}$ as investigated by Verkin et al. (1979), but now, due to the antı-ferromagnetıc ordering in $\mathrm{GdCu}_{2} \mathrm{~S}_{1_{2}}$, at much lower temperatures $\left(\mathrm{T}_{\mathrm{C}}=12 \mathrm{~K}\right)$. In tne $\mathrm{Tb}$ experiments, sharp maxima in the measured resistance signal have been ascribed to electron-magnon scattering, because as the energles where the peaks occur compare reasonably well with the structures in magnon density of states as obtalned from inelastic neutron-scattering experiments. However, it is not clearly understood why a maximum is observed in the resistance instead of in the voltage derivative of the resistance.

Very recently experiments have been performed on point contacts, made from magnetically dilute alloys, in order to study the exchange scattering of the electrons with magnetic impurities in the metal (Jansen et al., 1980 b). The experiments were done whth Au and $\mathrm{Cu}$ host samples with small concentrations (< 1 at. 8 ) of respectively $\mathrm{Mn}$ and $\mathrm{Fe}$. The point-contact 
spectra revealt an interesting structure, which corresponds to a maximum in the resistance at $\mathrm{V}=0$. An explanation of the observed phenomenon can be given by considering the scattering time for the interaction of the electrons with magnetic impurities (see formula (4.34)). In figure 30 we have given the measured differential resistance as a function of the applied voltage for a AuMn Junction. In the same figure we have also plotted the second derivative $\bar{d}^{2} v / d I^{2}$, which clearly shows the phonon structure,

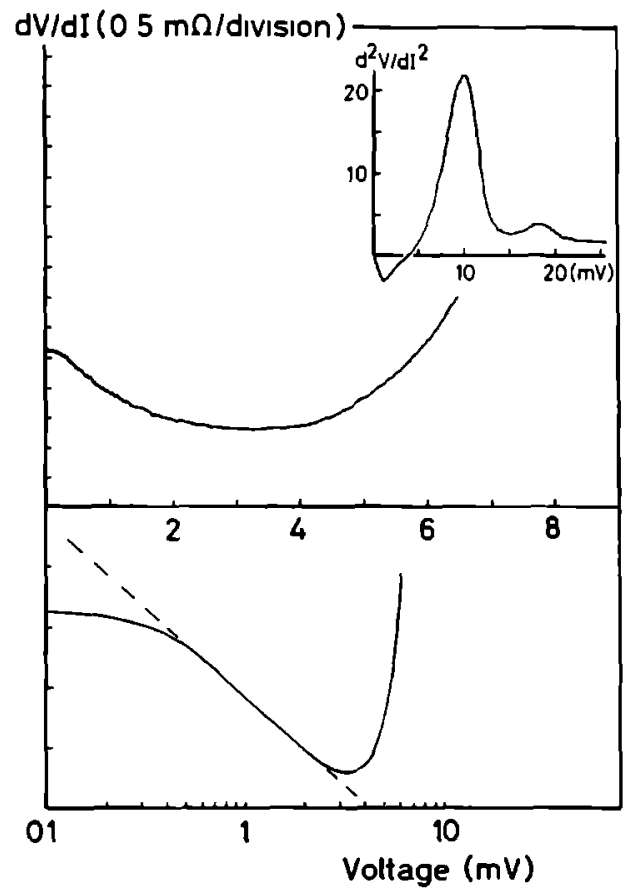

Fig. 30 Measured differential resistance dV/dI for a point contact of the magnetically dilute alloy $\mathrm{Au}-0.03 \mathrm{at.} \mathrm{z}$ Mn as a function of the applied voltage (linear and logarithmic scale). Resistance $R_{0}=2.1 \Omega$; temperature $T=1.5 \mathrm{~K}$. The dashed line in the bottom figure gives the logarithmic slope of the resistance. In the insert, the second derivative signal reveals the phonon structure of $\mathrm{Al}$.

Indicating that we are indeed dealing with point-contact spectroscopy with a backflow current. Heating effects can be neglected as we are still in the knudsen regime for the investigated point contacts, according to tne 
observed electron-phonon structure seen in the signal. Note the characteristic difference between tunneling and polnt-contact spectroscopy: In tunneling experiments performed on metal-insulator-metal films, structures as a function of the applied voltage have been observed, indicating a mininum in the resistance whicn is due to the coupling between electrons and magnetic impurities in the oxıde layer (Wyatt, 1964). This in drastic contrast with the maximun in the resistance as observed in the pointcontact experiments.

For a detalled analysis and understanding of most of the phenomena due to the exchange interaction in Kondo systems (1.e. bulk resistıvity, specific heat, susceptibility, etc.) a determination of the energy dependence of the scattering rate is important. Theoretically, the scattering time of an electron with energy $\mathrm{eV}$ above the Fermi level has been evaluated for a Kondo system in several ways (Suhl, 1973). The energy-dependent scatterıng tıme $\tau(\mathrm{eV})$ as given by Hamann (1967) reduces $1 . e$. in the limit $\mathrm{eV} \gg k_{B} \mathrm{~T}$ to

$$
\frac{1}{\tau}(\mathrm{eV})=\frac{\mathrm{c}}{\pi \mathrm{N}_{0}}\left\{1-\ln \left(\frac{\mathrm{eV}}{\mathrm{k}_{\mathrm{B}} \mathrm{T}_{\mathrm{K}}}\right)\left[\ln ^{2}\left(\frac{\mathrm{eV}}{\mathrm{k}_{\mathrm{B}} \mathrm{T}_{\mathrm{K}}}\right)+\mathrm{S}(\mathrm{S}+1) \pi^{2}\right]^{-\frac{1}{2}}\right\}
$$

where $T_{K}$ is the characteristic Kondo temperature of the dilute alloy ana $c$ the concentration of magnetic impurities with spin S. From the Hamann formula for the scattering rate, the temperature dependence for the bulk resistivity can be obtalned, which is formally similar to the voltage dependence of $\tau^{-1}(\mathrm{eV})$ in equation $(8.2)$. It is therefore reasonable to compare the point-contact data as a function of voltage qualitatively with bulk resistivity measurements as a function of temperature. Note that from a theoretical point of view, it is a colncidence due to the nature of the specific logarithmic terms in the expression for the scattering time that the temperature dependence of the resistivity and the energy dependence of the inverse of the scattering time behave similar.

The Kondo temperature for a AuMn-system is very low $\left(T_{K} \sim 10^{-13} \mathrm{~K}\right)$. Using $\left|\ln T_{K}\right| \gg 1$, equation (8.2) can then be simplified to $\tau^{-1}(\mathrm{eV})$ a $\left\{1-2 \ln (\mathrm{eV}) / \ln \left(\mathrm{k}_{\mathrm{B}} \mathrm{T}_{\mathrm{K}}\right)\right\}$. In figure 30 we have also plotted the measured point-contact resistance as a function of the voltage in a logarithmic scale. It can be seen that over a limited range around $1 \mathrm{mV}$ a logvbehaviour is observed. For higher voltages, the resistance increases due to the electron-phonon interaction, and at low voltages ordering effects 
and temperature smearing play a role in the rounding off of the resistance maximum. Similar ordering effects have been observed for concentrations $\gtrsim 0.02$ at. $\mathrm{Mn}$ in $\mathrm{Au}$ in bulk resistivity experiments (Loram et al., 1971). In figure 31 point-contact experiments for different Mn concentrations in the Au samples are shown. Upon increasing the Mn concentration, firstly a broadening of the resistance maximum at $\mathrm{V}=0$ occurs, and then the maxımum splits up in two maxima. Qualitatively the same behaviour has been observed in bulk resistivity experıments as a function of the temperature (Loram et al., 19/1). The experlments with more concentrated samples can

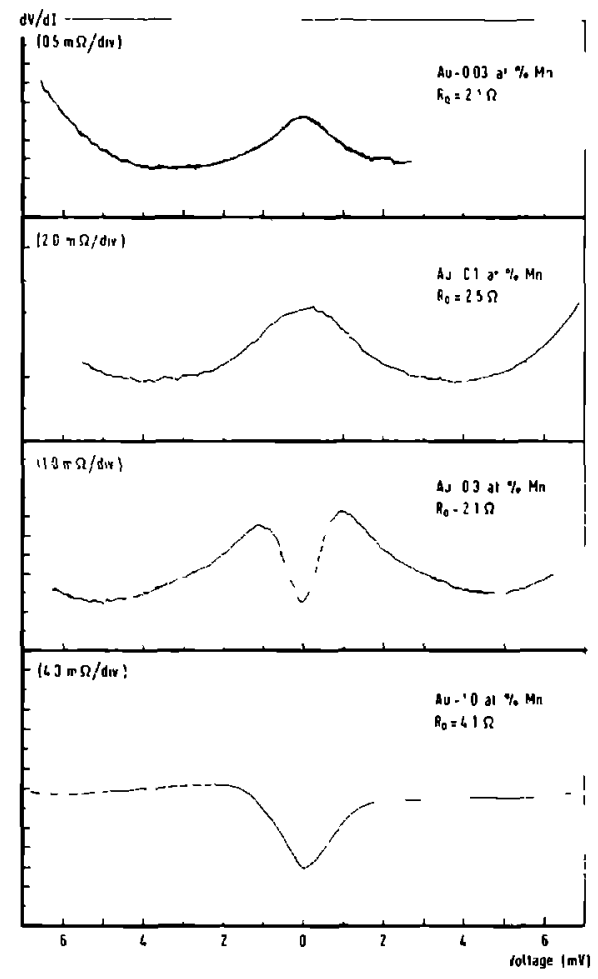

Fig. 31 Measured differentıal resistance dV/dI for hu point contacts witi aifferent concentrations of $\mathrm{Mn}$.

be described by orderıng, or equivalently, internal magnetic fields. This is clearly demonstrated in figure 32, where the resistance of a AuMn point contact is given as function of voltage in magnetıc fields up to $30 \mathrm{kG}$. Also in CuFe dilute alloys maxima in the resistance at $\mathrm{V}=0$ have been 


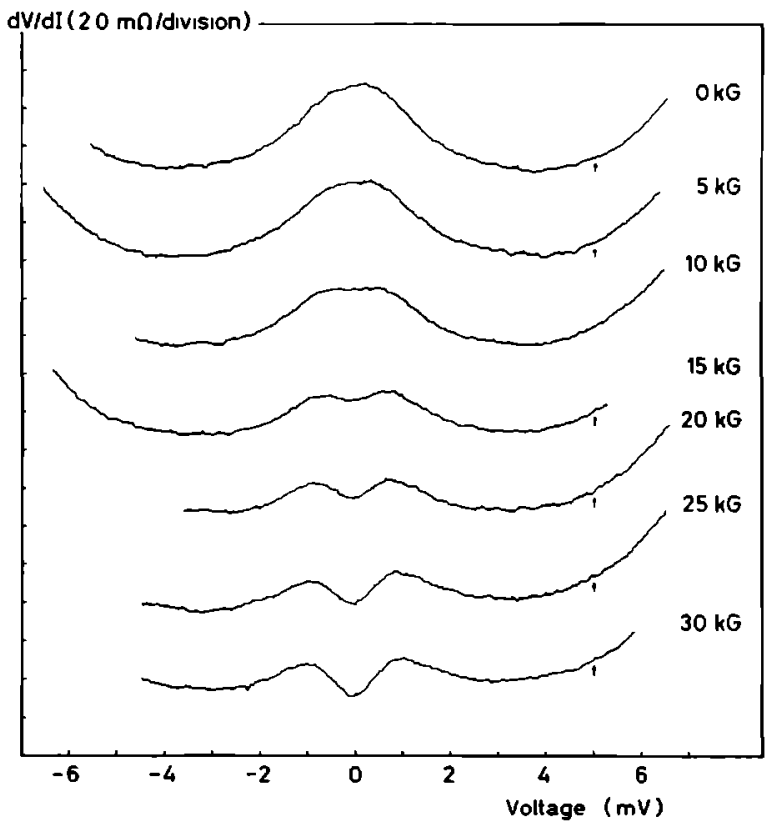

Fig. 32 Measured differential resistance dV/dI for a point contact of Au-0.1 at. $\%$ Mn $1 n$ an applied magnetic field (perpendicular to the contact area). Resistance $R_{0}=2.5 \Omega$, temperature $T=1.5 \mathrm{~K}$. The curves are shifted with respect to the reference point indicated by $(\uparrow)$.

observed (Jansen et al., 1980 b). For the CuFe case, the temperature of the helium bath used for the experiments lies below the Kondo temperature ( $30 \mathrm{~K}$ ). Therefore, for very small voltages, the unitarity limit giving the maximum in the cross-section for the elcctronic scattering might be roached. A first indication gives the experimental result with a flat part in the resistance below $0.5 \mathrm{mV}$ ( $20 \%$ of the Kondo energy $\mathrm{k}_{\mathrm{B}} \mathrm{T}_{\mathrm{K}}=2.6 \mathrm{mV}$ ). However, the bath temperature should be lowered even more to give clear evidence for an observation of the unitarity limit.

These first experimental results on ferromagnetıc metals and on kondo samples show convincingly that point-contact spectroscopy is a most promising and interesting tool for investigating the scattering mechanism of the conduction electrons in metals. Although this now spectroscopy has 
been applied most successfully until now mainly for the study of the electron-phonon interaction, it is quite clear that the method will be equally useful for other interaction mechanisms as well. However, one has always to keep in mind that in order to bring the conduction electrons out of equilibrium within a distance of the order of the mean free path, it is essential that the samples to be studied with point-contact spectroscopy have to have an energy dependent mean free path of the electrons which is at least of the order of the spatial dimensions of the contact. 
Adler J.G. and Jackson J.E. 1966, Rev. Sc1. Instr. 37, 1049. Blrgeneau R.J., Gordes J., Dolling G. and Woods A.D.B. 1964, Phys. Rev. $136 \mathrm{~A}, 1359$.

Carbotte J.P. and Dynes R.C. 1968, Phys. Rev. 172, 476.

Chaıkın P.M. and Hansma P.K. 1976, Phys. Rev. Lett. $\underline{36}, 1552$.

Cowley R.A., woods A.D.B. and Dolling G. 1966, Phys. Rev. 150, 487.

Danıno M., Kaveh M. and Wıser N. 1978, J. Phys. (Parıs) 39, C6-1046.

Das S.G. 1973, Phys. Rev. B 7, 2238.

Dolling G. and Woods A.D.B. 1965, Thermal Neutron Scatterıng, ed. P.A.

Egelstaff (New York: Academıc Press Inc.).

Dozler J.W. and Rodgers J.D. 1964, IEEE Trans. Mlcrowave Theory Tech.

$\underline{12}, 360$.

Frauenfelder H. 1962, The Mōssbauer Effect (New York: W.A. Bergamın Inc.). van Gelder A.P. 1978, Solıd State Commun. 25, 1097.

1980, to be published.

van Gelder A.P., Jansen A.G.M., Strassler S. and Wyder P. 1978, J. Phys.

(Paris) 39, C6-602.

van Gelder A.P., Jansen A.G.M. and Wyder P. 1980 a, to be published.

$1980 \mathrm{~b}$, to be published.

Gilat G. and Raubenhelmer L.J. 1966, Phys. Rev. 144, 390.

Grimvall G. 1976, Phys. Scr. 14, 63.

Hamann D.R. 1967, Phys. Rev. 158, 570.

Haymann B. and Carbotte J.P. 1971, J. Phys. F: Metal Phys. 1, 828.

1972, J. Phys. F: Metal Phys. 2, 915.

Holm R. 1967, Electric Contacts (Berlın: Springer Verlag).

Jansen A.G.M., Mueller F.M. and Wyder P 1976, Proc. 2nd Rochester Conf. on Superconductivity in $d-$ and f-band Metals, ed. D.H. Douglass (New York: Plenum).

- 1977, Phys. Rev. B $16,1325$.

- 1978, Science 199, 1037.

Jansen A.G.M., van den Bosch J.H., van Kempen H., Rıbot, J.H.J.M., Smeets

P.H.H. and Wyder P. 1980 a, J. Phys. F: Metal Phys. 10, 265.

Jansen A.G.M., van Gelder A.P., Wyder P. and Strāssler S. 1980 b, to be published.

Kleın J., Léger A., Belın M. and Defourneau D. 1973, Phys. Rev. B 7, 2336. 
Knudsen M. 1934, Kınetıc Theory of Gases (London: Methuen) .

Kulık I.O., Omel'yanchuk A.N, and Shekhter R.I. 1977, Fız. Nızk. Temp. $\underline{3}$, 1543 (Sov. J. Low Temp. Phys. 3, 740).

Kulık I.O., Shekhter R.I. and Omel'yanchuk A.N. 1977, Solıd State Cormun. 23,301 .

Kulık I.O. and Yanson I.K. 1978, F1z. Nlzk. Temp. 4, 1267 (Sov. J. Low

Temp. Phys. 4 , 596).

Lambe J. and Jaklevıc R.C. 1968, Phys. Rev. 165, 821.

Leppın H.P. and Wohlleben D.K. 1978, J. Less Common Metals 62, 303.

Loram J.W., Whall T.E. and Ford P.J. 1971, Phys. Rev. B 3, 953.

Lynn J.W., Sinıth H.G. and Nicklow R.M. 1973, Phys. Rev. B 8, 3493.

Maxwell J.C. 1904, A. Treatıse on Electrıcıty and Magnetısm (Oxford:

Clarendon) .

McMillan W.L. 1968, Phys. Rev. 167, 331.

MCMıllan W.L. and Rowell J.M. 1969, Superconductıvity vol. 1, ed. R.D.

Parks (New York: Marcel Dekker Inc.).

Omel'yanchuk A.N., Kulık I.O. and Shekhter R.I. 1977, Pıs'ma Zh. Eksp.

Teor. Fiz. 25, 465 (JETP Lett. $\underline{25}, 437$ ).

Parks R.D. 1969, ed. Superconductıvıty (New York: Marcel Dekker Inc.). Rowell J.M., McMlllan W.L. and Dynes R.C. 1973, A Tabulation of the

Electron-Phonor Interaction in Superconducting Metals and Alloys,

part I, Bell Tel. Labs., Murray Hı11, USA (unpublıshed).

Rowell J.M., McMrllan W.L. and Feldman W.L. 1969, Phys. Rev. 180, 658.

Shalov Yu.N. and Yanson I.K. 1977, Flz. Nlzk. Temp. 3, 99 (Sov. J. Low

Temp. Phys. 3,48 ) .

Sharvin Yu.v. 1965, Zh. Eksp. Teor. Fız. 48, 984 (Sov. Phys. - JETP 21, 655).

Smith H.G., Dolling G., N1cklow R.M., Yljayaraghavan P.R. and Wilkinson

M.K. 1968, Neutron Inelastic Scattering, Proc. Symp., Copenhagen

vol. I, p. 149 (Vienna: IAEA).

Stedman R., Almqvist L. and Nilsson G. 1967, Phys. Rev. 162, 549.

Suhl H. 1973, ed. Magnetısm vol. V (New York: Academıc Press).

Trofımenkoff P.N., Kreuzer H.J., Wattemanıuk W.J. and Adler J.G. 1972,

Phys. Rev. Lett. 29, 597.

Truant P.T. and Carbotte J.P. 1973, Can J. Phys. 51, 922.

Verkın B.I., Yanson I.K., Kulık I.O., Shklyarevskı O.I., Lysykh A.A. and Naydyuk Yu.G. 1979, Solıd State Commun. 30, 215. 
Walker C.B. 1956, Phys. Rev. 103, 547.

Wexler G. 1966, Proc. Phys. Soc. London 89, 927.

Wolf E.L. 1978, Inelastic Tunneling Spectroscopy, ed. T. Wolfram (Berlin: Springer) .

Wyatt A.F.G. 1964, Phys. Rev. Lett. $13,40$.

Yanson I.K. 1974 a, Zh. Eksp. Teor. Fiz. 66, 1035 (Sov. Phys. - JETP 39, 506) .

- 1974 b, Fiz. Tverd. Tela 16, 3595 (Sov. Phys. Solid State 16, 2337).

- 1977, Fiz. Nizk. Temp. 3, 1516 (Sov. J. Low Temp. Phys. 3, 726).

Yanson I.K. and Batrak A.G. 1978, Pis'ma Zh. Eksp. Teor. Fiz. 27, 212 (JETP Lett. 27, 197).

Yanson I.K. and Kulik I.O. 1978, J. Phys. (Paris) 39, C6-1564.

Yanson I.K. and Shalov Yu.N. 1976, Zh. Eksp. Teor. Fiz. 71, 286 (Sov. Phys. - JETP 44,148 ).

Young J.A. and Koppel J.U. 1964, Phys. Rev. 134, A1476. 


\title{
Direct measurement of electron-phonon coupling $a^{2} F(\omega)$ using point contacts: Noble metals
}

\author{
A $\mathbf{G}$ M Jansen, $\mathbf{F} \mathbf{M}$ Mueller, and $\mathbf{P}$ Wyder \\ Physics Laboratory and Research Institute for Moterials Lntversity of Nymegen. Toernooiveld. Nijmegen The tetherlands
}

(Received 30 December 1976)

\begin{abstract}
A new technique of forming tuny point contacts between normal metals is described By measunng the voltage denvative of the resistance of such contaus at $12 \mathrm{~K}$, structure is found which is consistent with bulkphonon densilies of states Similar results were reported recently by Yanson using a shorted film technique When interpreted, the observed structures yield electron-phonon coupling parameters in close agreement with literalure values
\end{abstract}

The measurement of the electron-phonon interaction has attracted considerable interest. ${ }^{1-3}$ Recently Chaikın and Hansma have estımated the electron-phonon coupling parameter $\lambda$ in $\mathrm{Al}$ and $\mathrm{Cu}$ using proximity-effect lunneling,' and Hoyt and Mota have estımated $\lambda$ 's for $\mathrm{Cu}, \mathrm{Ag}$, and $\mathrm{Au} u$ using concentration-squared exirapolations based on the McMillian equation and the superconducting critical temperature of $\alpha$-phase noble-metal-rich alloys. ${ }^{5}$ In this paper we report measurements of the voltage derivative of the resistance of junctions formed by tiny conlacts between two noble metals, which show deviations from Ohm's law. ${ }^{5}$ Structures are seen which coincide well with bulk phonon spectra, and when interpreted yield $\lambda$ 's close to those expected ${ }^{1-5}$ for noble metals. Some of our results have been presented qualitatively at the Rochester meeting. ${ }^{3}$

We were stimulated to try our experiments by the work of Yanson. ${ }^{7, \theta}$ He used shorted junctions formed from normal metallic films separated by an insulator. The measured junctions were in the normal state, elther because of the Dewar temperalure or because of the application of an external magnetic field. His $\mathrm{Cu}$ results are in excellenl agreement with those presented here. It occurred to us that if we could form tiny metallsc bridges from sharp points, the resulting junctions would, in the normal state, be Sharvin junctions, ${ }^{9}$ and perhaps be simpler to form and control than those formed from shorted films. The major potential disadvanlages of such junctions, compared with those formed from films involved questions of mechanical slability.

We formed a sharp t1p $\left(-\frac{1}{2} \mu \mathrm{m}\right)$ on a thin wire ("spear") through an electrolytic etching technique. This was mounted rigidly on a subassembly with a larger wire ("anvil"), cooled to $1.2 \mathrm{~K}$ and carefully pressed into the anvil. The separation was first crudely adjusted using mechanıcal differential screws and then finely adjusied, using a piezoelectric substage. We estimate that the anvil-spear separation could be controlled this way to better than $10^{-6} \mathrm{~cm}$. An individual point contact was made and broken many tımes untıl a stable and reproducible value of resistance was achieved. Using the piezoelectric lever arrangement, the resistance of noble-metal point contacts ranged from 2.5 to $60 \Omega$. Once formed, the point contacts had stable resistances for 3-4 h. The first and second de rivative $\left(a V / \partial I\right.$ and $\left.\partial^{2} V / \partial I^{2}\right)$ were recorded using conventional ac modulation, phase-sensitive detection, and lock-in techniques, similar to those used in superconducting tunneling-spectroscopy The modulation voltage was small, typically $300 \mu \mathrm{V}$ The resistance $R(V)=\partial V / \partial I$ was smooth as a function of voltage, but not constant. For applied voltages grealer than about $30 \mathrm{mV}$, the resistance of all such junctions increased linearly with voltage; below $30 \mathrm{mV}$, the behavior was roughly parabolic. Thus the resistance of the junctions was distinctly non-Ohmic in both regimes, but metallic in the sense that it was a monotonically increasing function of voltage.

For a clear discussion on this point we refer to Rowell et al. ${ }^{2}$

The voltage dependence of various $(\partial R / \partial V)$ $\left[\equiv\left(\partial^{2} I^{\prime} / \partial I^{2}\right) /\left(\partial V^{2} / \partial I\right)\right]$ [ell into three distinct types: Those presented here in Fig. 1 ("normal"); those which had a large second derivatuve peak at less than 5-mV bias ("anomalous"), those which showed structure similar to that in Fig. 1, but with a rapid oscillatory modulation of order $1 \mathrm{mV}$ ("multıple-junction"). Similar elfects were seen by Yanson and Shalov ${ }^{B}$ in their film experiments. In our experiments the three types divided roughly as $70 \%, 25 \%$, and $5_{c}^{\prime \prime}$ of the formed junctions, respectively. In Figs. 1(a)-1(c) typical results are glven for $\partial R / \partial V^{\prime}$ of $\mathrm{Cu}, \mathrm{Ag}$, and $\mathrm{Au}$ as a function of voltage for "normal" junctions. In all cases $R(V)$ was nearly a symmetric function of $V$ and $d R / \partial V$ an antisymmetric function of $V$. Two pieces of structure are seen at voltages less than $30 \mathrm{mV}$, above this value $\mathrm{J} R / \mathrm{oV}$ is constant. In the same figure literature values are given for the phonon density of states $F(\omega)$ for $\mathrm{Cu},{ }^{10} \mathrm{Ag},{ }^{11}$ and $\mathrm{Au},{ }^{12}$ obtained from neutron scaltering experıments. The 


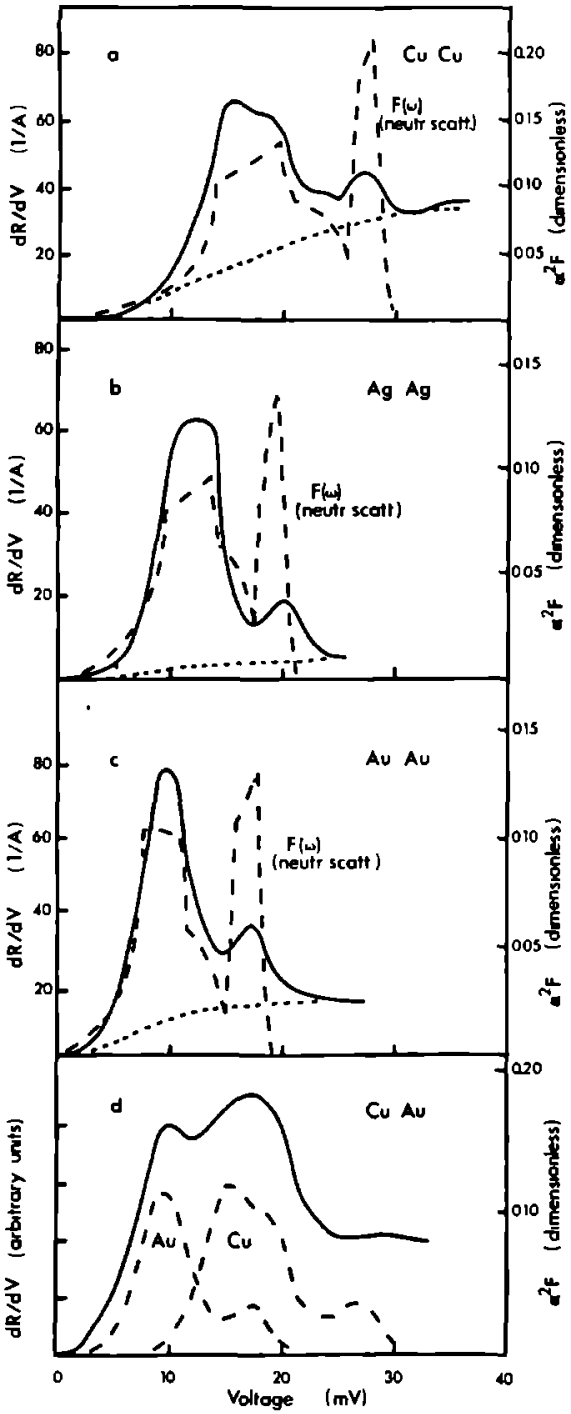

FIG 1 Measured voltage derivative of the resistance of point contacts as a function of applied voltage of (a) $\mathrm{Cu}-\mathrm{Cu}$, (b) $\mathrm{Ag}-\mathrm{Ag}$, (c) $\mathrm{Au}-\mathrm{Au}$, and (d) $\mathrm{Cu}-\mathrm{Au}$, plotted as the solid lines (deft-hand scale) The right-hand scale is derived through Eqs (2) and (3) In (a), (b), and (c) the backround functions are shown as short-dashed curves, and phonon density of states from Ref 12 as long-dashed curves The dotted line in (b) shows the small measured anomaly at zero bias in (d) is plotted as long-dashed curses the difference between the measured solid curve and the bachground function for Au and $\mathrm{Cu}$, respectively cancidence of the low voltage peak of our results with the transverse phonon peak is excellent The higher peak coincides well with the longitudinal peak, with a small disagreement in the case of $\mathrm{Ag}$. We belicve, therefore, that bulk phonons play a significant role.

The resistivity ratio (RR) of our samples given in Table I is low and the resistance at zero voltage $\Omega_{0}$ is high compared with previous experiments on melallic contacts. Estımatıng the contact radius $a_{N}$ from $a_{N}=\rho / 2 \Omega_{0}$ ( $\rho$ is the resistivity of the metal, Maxwell ${ }^{13}$ derived this formula using Ohm's law) and the data of Table I we find values smaller than $02 \mathrm{~A}$. These are far smaller than our estimates of the residual impurity scallering length $l_{\text {amp. }}$ Thus the flow of electrons through the contact orifice would have additional impedance due to the Knudsen ${ }^{14}$ effect. Wexler ${ }^{15}$ has given a detalled treatment of the resistance in the case where the orifice size $a$ is small compared with the scattering length $l$. He gives

$$
R_{\text {Int }}=\Gamma(K) / 2 \sigma a+4 K / 3 \pi \sigma a,
$$

where $\Gamma$ is a slowly varying lunction of the Knudsen number $K=l / a$ which takes the value 1 at $z$ ero and $\frac{\theta}{12 \theta} \pi^{2}$ at high $K$ and $\sigma=1 / \rho$ the conductivity of the bulk material. The first term in this formula is dominant for small $K$ and similar to the resistive formula of Maxwell ${ }^{13}$ The second lerm dominates for high $K$ and is similar to the formula of Sharvin. 9 The regime of high $K$ is of interest here and the radı given in Table I are found from $a^{2}=4 \rho l / 3 \pi \Omega_{0}$. These radıl are much larger than our Maxwellıan estimates, but the condition $K \gg 1$ is sill fulfilled. We assume that only the scattering length $l$ depends on the voltage $V$ (or energy eV). Thus since $\rho l$ is independent of $l$ and $\Gamma$ a slowly varying function of $K$, the derivative $\partial R / \partial V$ is given by

$$
\frac{\partial R}{\partial V}=\frac{\Gamma \rho l}{2 a} \frac{\partial}{\partial V} \frac{1}{l(e V)}
$$

and the total scattering length by $1 / l=1 / l_{\text {Imp }}+1 / l_{\text {cp }}$, where

$$
\begin{aligned}
& \frac{1}{l_{\text {.p }}}= \frac{1}{v_{F} \tau(e V)} \\
&=\frac{2 \pi}{{ }^{2} \hbar} \int_{0}^{\infty} d \omega \alpha^{2} F(\omega)\left[2 N_{0}(\omega)+1-f_{0}(e V-\omega)\right. \\
&\left.\quad+f_{0}(e V+\omega)\right]
\end{aligned}
$$

is the energy-dependent phonon-emission length at energy $e V$, and $\alpha^{2} F$ the frequency-dependent electron-phonon coupling constant Thus at low temperatures we obtain directly $\alpha^{2} F$, given as the right-hand scales of Fig 1 . In the theories of electron-phonon coupling reviewed by Zavarıtskuı and Grimvall, ' $1 / l_{\text {ep }}$ becomes constant at high en- 


\section{UIRECT VEASURF MEVT OF FIECTROY IIOYON COLPLING}

TABLf I F xperimental parameters for the four polnt conticts plotted in $F_{1 g}$ I $\Omega_{0}$ is the junction resistance at zero voltage, $l_{\mathrm{Imp}}$ the $1.2-\mathrm{K}$ resistive scatte ing le ngh, $l_{\text {ep }}$ the 30 -mev electron-phonon emission length, a the orifice radius, and $K$ the Knudsen ratio $l / a \quad \lambda_{p}$ is tuice the integral of the deisucd $\alpha^{2} F / \Delta$

\begin{tabular}{|c|c|c|c|c|c|c|c|c|c|c|}
\hline & RIR & $\begin{array}{l}\Omega_{0} \\
(\Omega)\end{array}$ & $\left(10^{-11} \stackrel{\rho l}{\Omega \mathrm{cm}^{2}}\right)$ & $\left(10^{-l_{0 D}} \mathrm{~cm}\right)$ & $\underset{\left(10^{-4} \mathrm{~cm}\right)}{l_{\mathrm{cm}}}$ & $\begin{array}{c}a \\
\left(10^{-8} \mathrm{~cm}\right)\end{array}$ & $K$ & $\lambda_{6}$ & $\lambda_{\mu}$ & $\lambda_{P}$ \\
\hline $\mathrm{Cu}-\mathrm{Cu}$ & 71 & 57 & 071 & 089 & 325 & 727 & 96 & $014 \pm 0 \quad 03$ & 016 & 014 \\
\hline $\mathrm{Ag}-\mathrm{Ag}$ & 43 & 163 & 091 & 101 & 326 & 549 & 140 & $010+004$ & 016 & 015 \\
\hline$A u-A u$ & $2 J$ & 320 & 104 & 116 & 125 & 371 & 162 & $014 \pm 005$ & 021 & 016 \\
\hline $\mathrm{Cu}-\mathrm{Au}$ & 35 & 25 & 088 & $\cdots$ & 251 & 1222 & $\cdots$ & $\cdots$ & $\cdots$ & $\cdots$ \\
\hline
\end{tabular}

ergy, 1 e, $\left(\partial / \partial_{e} V\right)\left(1 / l_{c_{1}}\right)-0$. We see that our results contain a phonon-emission effect, and another smooth ("background") effect. We have approximated the second effect through the dashed functions presented in Fig. 1, given by

$$
B(V)=C \tanh ^{2}\left(1.5 \mathrm{eV} / k \theta_{0}\right) \text {, }
$$

where $C$ is a constant fitted at $30 \mathrm{mV}$. To compare with other experiments, we integrate to find the $\lambda_{p}$ 's, $\lambda_{c}$ is laken from Grimvall' and $\lambda_{y}$ from Hoyt and Mola," given in Table I. On the basis of the close coincidence of the structure of Fig. 1 with phonon structures, and the agreement with literature values of $\lambda$, we conclude that the voltage derivative of the resistance of small point contacts provides a simple and convenient method to measure $\alpha^{2} F(\omega)$ in normal metals. The same conclusion was reached by Yanson in his experiments using evaporated films.

We will briefly give some other experimental results and conclusions

(1) Figure 1(d) tests the possibility of dissimilar junctions-here with a "spear" of $\mathrm{Cu}$ and "anvil" of Au. The results suggest that the effect is additive - as was given by Wexler, ${ }^{15}$ Eq. (69).

(11) Usıng anvils of single crystals, preliminary experiments seem to show that the results do not depend on the exposed crystalline face. The form of the variational function of Wexler suggests that the half width at half maximum of the electrons which pass the orifice is $45^{\circ}$. This is $82^{\circ}$ of $54.7^{\circ}$, the angle between [100] and [111].

(iii) Plotting all our experimental data, the ampli- tude of the first peak scales as $\Omega_{0}$ to the 0.36 \pm 013 power, this is consistent with the analysis above ( 05 power) The size of the residuals suggest that the internal consistency of the data is of the order of $20 \%$.

(1v) Yanson has defined $\gamma$, the ratio of the "background" to the first peak height. ${ }^{B} \gamma$ shows a dependence as $\Omega_{0}$ to the power -0.54 for Yanson's Cu data and -053 for ours. Thus $B$ does not depend on $\Omega_{0}$

(v) $B$ depends on the residual resistance ratios $\mathrm{RR}$ of the anvils as the power -052 .

(vi) The ratio of the $\alpha^{2} F$ 's of Fig. 1 to the related $F(\omega)$ 's shows that $\alpha^{2}$ is strongly $\omega$ or energy dependent Transverse phonons couple to the electron gas aboul four times more effectively than longitudinal phonons in noble metals This is consistent with the results and conclusions of a theory of Das, who first suggested ${ }^{16}$ in \& quantitative way, that $d$ electrons were important and strongly umklapp coupled to transverse phonons in noble metals. It is likely that the same is true in transition metals-or "transition-metal compounds."

\section{ACKNOWLEDGMENT}

We wish to thank H. W. H. M. Jongbloets for help in setting up the experiments and A. P. van Gelder for helpful theoretical discussions. This work was performed as part of the research program of the Stichtıng voor Fundamenteel Onderzoek der Materie (FOM) with financial support from the Nederlandse Organisatie voor Zuıver Wetenschappelijk Onderzoek (ZWO)
${ }^{1} \mathrm{~N}$ V Zavaritskir, Usp Fiz Nauk $\underline{108}, 241$ (1972) [Sov Phys - Csp 15, 608 (1973)l, G. Grimvall, Phys. Scr. 14, 63 (1976)

2J M Rowell, $W$ L Mc villan, and if $L$ Feldman [Phys Rev 180, 658 (1969)] and J G Adler [Phys Lett A 29, $\overline{675}$ (1969)] flrst observed normal metal phonons in tunnel junctions

${ }^{3}$ Second Rochester Conference on $d$ - and f-band Superconductors, edited by D H Douglass (Plenum, New
York, 1976), and references therein

${ }^{4} \mathrm{P}$ M Chaskin and P K llansma, Phys Rev Lett 16 , 1552 (1976)

${ }^{5}$ R F Hoyl and A C Mota, Solid State Commun 18 . 139 (1976)

'I Stone, Phis Ret 6, 1 (1998), P W Bridgman, tbid 9, 269 (1917); R Holm, Flectric Contacts (sprıngerVerlag, Berlin, 1967)

${ }^{7} \mathrm{I} K$ Yanson, Zh Eksp Teor. Fiz. 66, 1035 (1974) 


\section{A G M JANSFN F Y VUFLLER ANDP WYDFR}

[So, Phys -JE IP 39, $506(1970)]$

${ }^{8} \mathrm{~K}$ Yanson and $\mathrm{Yu} \mathrm{N}$ Shalov, $7 \mathrm{~h}$ F ksp Teor Fiz 71,286 (1976) [Sor Phys -JETP 44, 148 (1976)]

'A Shariun junction is a metallic junction in the extreme Knudsen regime $(l / a>1)$ Yu $V$ Sharvin, Zh Fksp Teor Fiz 48 984 (1965) [Sol Phys -JETP 21,65 . (196.)]

${ }^{10} \mathrm{R}$ M Nicklow, G Gilat, H G Smith, L J Raubenheimer, and $M$ K Wilkinson, Phys Rev 164, 922 (1967)
${ }^{11} \mathrm{~W}$ A Kamitakahara and $\mathrm{B} M$ Brockhouse, Phys Iett A 29, 639 (1969)

${ }^{12} \mathrm{~J}$ IV Lyn, H G Smith and $\mathrm{R} M$ Nicklow, Phys Rev B 8, 3493 (1973)

${ }^{13} \mathrm{~J}$ C Maxwcll, A Truatise on Electricty and Vagnetesm, 1891 (Dover New York, 19,4)

${ }^{14} \mathrm{M}$ Knudsen, Kinetic Theory of Gases (Miethuen, London, 1934)

${ }^{13} \mathrm{G}$ Hexler, Proe Phys Soc Lond 89, 927 (1966)

${ }^{16} \mathrm{~S}$ G Das, Phys Rev B $7,2238(1 \overline{973})$ 


\section{Normal Metallic Point Contacts}

\author{
The (nonohmic) resistance of tiny metal contacts \\ shows new structure at metal phonon energies.
}

A G M Jansen, F M Mueller, P Wyder

With the widespread use of minatur tzed semiconducting devices (diodes transistors, and integrated circuit chips), new problems arose in attaching stable metrilic leads Although the currents that flowed through the metallic leads were small-iypically of the order of a few millamperes-the contacts were so small that the resulting current densities were enormous-as large as $10^{6}$ amperes per square centimeter Such high curtent densitues were well above anything encountered in the laboratory or in tech-

Impuritics become wedker and neakerand eventually break These technical problems are now well understood, but the solution has involved d wide variety of disciplines

\section{Background: Large Contacts}

The general problem of understanding electncal contacts is quite old it first arose with the advent of rotating electrical machinery in the early 1890 's The

Summary The measured vollage derivative of the nonlinear resistance of tiny point contacts can be separated into a phonon-emission efilect $\left(\alpha^{2} F\right)$ and an analytic functional form (background effect) The $\alpha^{2} F$ 's show structure coincident with bulk phonon densities of stales Values of the integral of $2 a^{2} F / \omega$ are closely related to literature values The background effect is related to the impurity concentration of the matenals

nology before In such a regıme, new phenomena, which in the ordinary world are very tuny, become important All metallic crystals contain impurities, vacancies, or dislocations In a strong cur rent density (or equivalently, a sirong electnc field), these defects force electrons to stream around them and hence exert forces on them, they move or diffuse, driven by the electron "wind " This process is now called electromigration ( $I$ ) When such diffusion takes place in constant strong fields for a long tıme, the defects migrate and become trapped-for example. at the metal-semiconductor inierface Mechanically, contacts with piled-up vacancies and SCIENCE VOL $19910 \mathrm{MARCH} 1978$ classical solution in the ohmic regume was given by Maxwell (2) by solving Poisson's equation for the electrical potential $V$ as function of coordinate $\vec{r}$

$$
\nabla^{2} V(\vec{r})=0
$$

in oblate spherical coordinates Cuntours of constant potential of current flowing through a constricting circular orifice of radius $a$ are given by

$$
V(\vec{r})= \pm V_{0}\left[1-\frac{2}{\pi} \arctan (1 / \xi)\right]
$$

where the oblate sphencal coordinate $\xi$ may be found from the more familar spherical polar coordinates $r$ and $z$ by solving the implicit equation $r^{2} / a^{2}=$ $\left(1+\xi^{2}\right)\left(1-z^{2} / \xi^{2} a^{2}\right)$ The two signs refer to the two sides of the circular orifice the geometry has cylindncal symmetry The resislance of such a conlact may be found by dividing the total voltage drop (here $2 V_{0}$ ) by the total current flowing through any contour of constant voltage For simplicity, we choose the contour $v(\vec{r})=0$. that 15 , the onfice Itself ( $\xi=0$ ) The current $/$ is given (in c) lindrical coordindtes) by an integral of the current densily $\vec{J}$ over the area $\vec{D}$ of the orifice

$$
\begin{aligned}
I & =\int d \dot{J} \vec{J} \\
& =\int_{a}^{2 v} d \varphi \int_{0}^{a} r d r \sigma\left(\begin{array}{l}
\partial V \\
\partial z
\end{array}\right)_{a=0}
\end{aligned}
$$

where $\sigma$ is the conductivity and $a V / a z$ is the $z$ component of the electric field $\vec{E}$ The Maxwellian resistance (2) of the contact is then given by

$$
\boldsymbol{R}_{\mathrm{V}}=\frac{\rho}{2 a}
$$

where $\rho$ is the resistivily $(\equiv 1 / \sigma)$ Equation 4 has been verihed ( 3 ) to an accuracy of about 1 percent for a uide variety of "practical" contacts There are also many exceptions ( $O$ )

\section{New Phenomena in Small Contacts}

In this article we focus on some interesting exceptions that have been examined $(4,5)$ at Nijmegen In these expenments the contact radu are made so small ( -40 angstroms) that the current densitıes are orders of magnitude $\left(10^{10}\right.$ to $10^{\prime \prime} \mathrm{amp} / \mathrm{cm}^{2}$ ) larger than those previously considered We were stimulated to try such expenments by the work of Yanson (6), who used a technique that depends on puncturing two metallıc films, separated by a thin $(100 \AA)$ insulating layer, with a burst of voltage coupled through a high resistor It oc-

A $G M$ Jansen is a graduate student and $F M$ Vueller and $P$ Wyder are professors of physics at the Physics I aboralory University of Nijmegen Nojmegen Netherlands All three are connected with the Suchung voor Fundamenteel Onderzoel der Matene (Foundation for Fundamental Research on Matter) This article is an English trangletion of an article wnilen for the Nederiands Tudschrifs voor Nafuurkunde 
curred to us that if we could form such tıny metallic bridges from sharp points the resultung junctions might be simpler to form and control than those formed from shorted films The major potentid disddvantages of poinl contacis involved questions of mechanical stability

Expermental techmoue We formed a sharp lip (of raduus - 1/2 micrometer) on d thin wire ( spear ) by an electrolyuc etching technique (7) This wals mounted rigidly on a subassembly with a larger wire ( anvil) cooled to $12^{\circ} \mathrm{K}$ and the spear anvil separation w is carefully ad justed On the bass of the results dis cussed below we believe that within the etched lip of the spear there are micro scopic regions which on this scale are Iarge conical mountains rising 100 to $200 \mathrm{~A}$ above the average plane of the IIp Uiually one of these is Jomınant ac Iually touches first and forms the metal lic resislive junction we measure The separation is first crudely adjusted by means of differential screws and then finely adjusted by using a piezoeleciric substage We can control the separation to better than $10^{\circ} \mathrm{cm}$ by means of the piezoelectnc lever arrangemen

The experimental apparatus is shown In Fig I The resistance of the point con tacts formed in noble melals at a low temperature (1 $2^{\circ} \mathrm{K}$ ) is quite high-about 10 ohms If we estumate the radus $a_{Y}$ of such a contact from the Maxwell equ i Ion ( $\mathrm{Eq} \mathrm{4)} \mathrm{we} \mathrm{find} \mathrm{the} \mathrm{IIny} \mathrm{value} \mathrm{of}$ about 02 A This is far smaller than both the classical resistive scattering length / (about $10^{4} \mathrm{~A}$ ) and the qu intum mechan cal de Broglie wavelength 1 (ahout 5 A) Moreover the medsured contact resist ance is distunctly nonlinear or non ohmic

vonlinear contacts In Fig 2 we show the voltage dependence of the current and the first and second denvatises of a nonlınear $I v$ characleristic curve for a point contact of copper whose resist ance was $57 \mathrm{ohm}$, at a voluge near ze ro [The medsunng lechnique involved phase sensituve detection of first and sec ond harmonics similar to that used in tunnel junciion spectroscopy of super conduciors (8) J Figure 2 is a direct pho tograph of the output of an i i plotter The (dynamical) resistance (curve b) is nearly an exacily symmeiric funcion of voltage the nonlınear second derivaluve (curve c) nearly an anisymmetric func tion The broad peaks in curve $c$ al \pm is and \pm 27 millivols are reproducible from sample to sample and conlact to contacl The small structure nedr zero bias and the ndrrow dip at $+17 \mathrm{mV}$ are electrical noise The tiny ripple seen at $>30 \mathrm{mV}$ is the intrinsic mechanical and elecincal noise in the experiment New phenomend have arisen because of the small size high current density and high electric field of the point con lacis

\section{Scattering Length versus Radius}

Let us consider the classical scattering length question first The problem of the flow of classical elecirons of large scall terıng length / through a sm all orifice iesembles a problem in gas kınetic theo r) (9) As the pressure on a gas vessel is lowered for example by pumping through a small hole into a vacuum the mean free path / between collisions in creases Eventually the path length will be larger than the orifice ridius a One then no longer has diffusive flow and the gas molecules penetrate the onfice bal lislically This problem wals first conid ered by Knudsen (10) in 1909 and is now called the Knudsen effect The different regumes are characterized by the Knud sen ratio $k-1 / a$

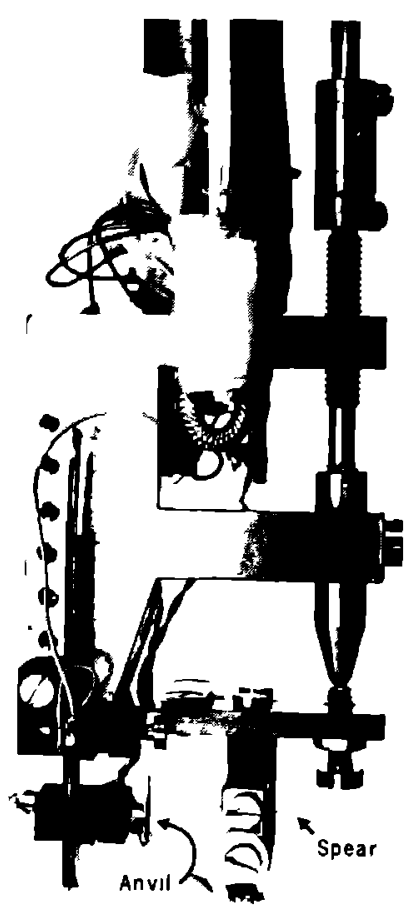

Fig I Experimental apparatus The spear on the right is moved into the anvil on the left by means of differenlial screws and a piezoelectric lever arrangement The separa IIon can be controlled to betler than $10^{\circ} \mathrm{cm}$
In the case of electrical contacts the major effect (In the regime of high Knud ien ratios) is to cause a steep voltage gradient or high effective electnc field to develop in the contact region Crudely then in this regime the contact serves as a device to inject electrons of high ki netic energy from one metal into anoth er but with a carefully controlled excess kinetic energy or velocity This excess velocily is given by $\mathrm{sl}= \pm \mathrm{c} V / \mathrm{p}_{\mathrm{r}}$ where the sign depends on whether the partucles passing through the orifice are holes or electrons, $c$ is the electric charge $V$ is the voltage drop across the contact and $p_{r}$ w the Fermi momentum The excess current $/$ is proporional to the cument density tumes the ared or nc $J i \pi a^{2}$ where $n$ is the partucle density Calculatung the restulance as $V / I$ we find $R=p_{r} / n c^{2} \pi a^{2}$ In the Drude theory of the electron gas the quantity $p_{r} / n c^{2}$ is equal to $\rho /$ the resistivity limes the ef fectuve scaltering length In the high $K$ regime the resistance of an electrical contact has been estımaled by Sharvin (II) as

$$
R_{\checkmark}-\frac{\rho K}{4 a}=\frac{K}{2} \quad R_{\mathrm{G}}=\frac{\rho l}{4 a^{\prime \prime}}
$$

(Note that in this form the resistance is quadratically dependen on the inverse of $a$ ) In our experiments the Knudsen resistance is larger than the Maxwell re ststance by the factor $K / 2$ or dbout 100 Using the Sharvin formuld we find the (larger) eslumate for $a$ of about $40 \mathrm{~A}$

Qummum effech Thus a is much larger than the de Broglie wavelength to that direcl quantum mechanical Fresnel (edge of the hole) interference phenom end (12 p 161) would be weak and would be visible only if a large voltage (about $600 \mathrm{mV}$ ) were impressed on the contact However if two or more of the

mountuins on the etched tip were of roughly equal height but spaced far apart then It would be posible to see the quanium mechanical analog of Young (double slit) interference (/2 p 114) If the two mountans were spaced $600 \mathrm{~A}$ upart then by the Hessen berg relation an extra momentum $p$ of aboul $10^{22}$ gram centumeter per second would be needed or an excess energy of about $05 \mathrm{mV}$ for free electrons to see interference

In a few of our formed contacts we see exactly the same emission phenome nd as will be discussed below bul with d 60 percent (height) modulation d d fre quency of about $075 \mathrm{mV}$ We interpret these ds quantum interference effects due to multiple junctions Such effects were also seen in the expenments of Yanson (6) 


\section{Interpolation Formula}

Between the two regimes-low $A$ (Maxwell) and high $A$ (Sharvar) one need an interpolation formula which system Itic ils tre its the probler ind yields these exlueme limits Wexler ( $J$ i) has given such a formula hased on 1 ,al lational solution to the nonlocal Boltz mann equation He iransformed the prohlem of electron flow through dn ori fice into the problem of an emıting disk The iransformation is similar to th it used by Babinet in treating the analogous problem of the equivalence of icrecns and holes in geometnc optics (12 p 147) Wexter s interpolation formula is

$$
R_{\mathrm{int}}=\frac{I(K)}{2 \sigma a}+\frac{4 K}{3 \pi \sigma a}
$$

where $I(R)$ is a slouly varying function of $A$ and lakes the value 1 for $\Lambda-0$ and $9 \pi^{2} / 128$ for large $A$ The first term is like the Maxwell resistance the second like the Sharuin resistance In our exper ments we take the partial denvative of the contact resislance with respect to voltage I We assume that the orifice radius is independent of voltage and the scditering length is dependent on voltage (or energ) ( $b$ ) The combination $k / G$ in the Shatvin like term is independent of voltage since $/ / \sigma$ is independent of the scattering length Thus only the first term enters the partial derivative

$$
\frac{\partial R}{\partial V}=\frac{I(k)}{2 \sigma a} \frac{\partial}{\partial V}\left(\frac{1}{(e V)}\right)
$$

(Note that the siructureless second term in Eq 6 serves ds a senes ballast resis tor to establish the voltage drop on the contact The first term is the actuve one ) The total scattering length / is given by

$$
\frac{1}{l}=\frac{1}{l_{1 \mathrm{mD}}}+\frac{1}{l_{\mathrm{ep}}}
$$

where $I_{\text {Imv }}$ is the scattenng length due to impunties and where $l_{\text {ep }}$ the electron phonon emission length is given by

$$
\begin{aligned}
& \frac{1}{l_{e \nu}}=\frac{1}{r_{r} \pi(e V)}=\left.\frac{2 \pi}{v_{r} h}\right|_{n} ^{x} d \omega \alpha^{2} \Gamma(\omega) \times \\
& {\left[2 N_{n}(\omega)+1-f_{1}(\omega)-(V)+f_{0}(\omega+e V)\right]}
\end{aligned}
$$

where $\downarrow$, is the Ferms velocity $\alpha^{2} f(\omega)$ the electron phonon coupling $1 / \tau(e b)$ the energy dependent emission rate and h Planck s constant over $2 \pi$ Equation 9 is the condensed matter analog of Fer mis second golden rule The matrix element squared is $\alpha^{2}$ the coupling constant and $f(w)$ is the phonon density of states The thermal Bose $\left(\wedge_{11}\right)$ and Fer

T ible 1 Fxperiment al parameters for the point contacts of $\mathrm{Fig}_{\mathrm{g}}$ Symbols $\boldsymbol{R}_{n}$ resislance of

\begin{tabular}{|c|c|c|c|c|c|c|c|}
\hline $\begin{array}{l}\text { Mate } \\
\text { rual }\end{array}$ & $\begin{array}{c}k \\
\text { rohm। }\end{array}$ & $\begin{array}{c}k \theta \\
(\pi, c V)\end{array}$ & $\left(10^{a} \mathrm{~cm}\right)$ & $\kappa$ & $\lambda$ & $\lambda_{n}$ & $\lambda_{1}$ \\
\hline$c u r u$ & $s^{-}$ & 992 & 727 & 9 & $(14=003$ & 016 & 014 \\
\hline $\mathrm{Ag} \mathrm{Ag}$ & 16 & 191 & 54 y & 140 & 0100004 & 016 & 019 \\
\hline Au Au & 320 & 140 & 371 & 162 & $014+005$ & 021 & 016 \\
\hline
\end{tabular}
the point cuntact 1 gero olt ige $L A$ Debycenergy a radius of the contact determined from $\left.\boldsymbol{R}(4) \pi(w) d^{2}\right) \boldsymbol{K}$ Knudien number $\lambda_{4}$ electron phonon $\Gamma$ irdmeter recommended by (srim

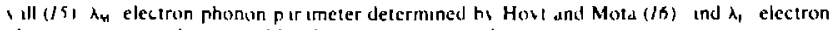
phonon parimeter deteimined hy the point contact technique

mi ( $\left.f_{0}\right)$ faclors enter because in con densed matter one needs to thermody namically average over the sistem At low temperatures as in our expenments the Bose tactor vanishes and the I ermı factors are nearly step funcions Taking the vollage derivative these hecomc Dirac delta functions centered at $\omega= \pm e l$ In the low tempelature limit only the one dt $\omega-p l$ contributes

\section{Experumental Results}

The dald for copper plotted in Fig 2 curve $c$ show hoth a slight dsymmelry (about I 0 ) and a sight posttive shift In the signd $W_{c}$ attribute the dsymme trv to a d c voltage bias due to the ther mal voltage over the coaxtal leads to the point contact at $12^{\circ} \mathrm{K}$ This small volt age shift is about $05 \mathrm{mV}$ We interpret the slight positive shift as due to first har monic leakage into the second hairmonic channel ds well ds tunneling ledkdge in

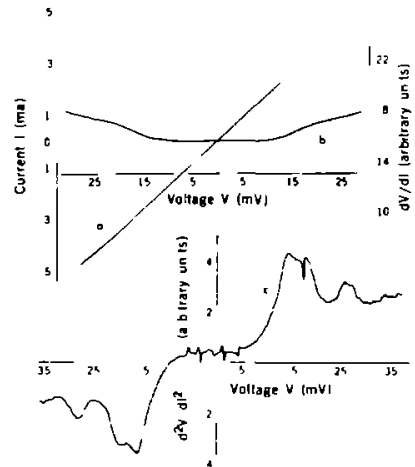

Fig 2 (left) Photograph of the recorder sheet with (curve a) current / (curve b) first denva tive $d V / d /$ and (curve c) second denva tive $d^{2} l d l^{2}$ plotted dganst applied volt age Fig 3 (roght) The measured voltage denvative of the resistance of point contacts di function of applied voltage for (d) $\mathrm{Cu}$ (b) $4 g$ and (c) Au is plotted as solid lines (left hand icale) The nght hand scale is the denved value for $a^{2} r$ The background functions are shown as shor dashed curves and the phonon densily of st thes as long dashed curves the contact itself [ which is even ( 8 ) in the second derisative] The continuous data were digitalized $I-D(\omega)]$ on a gnd of 05 mV A new energv scale shifled by $\Delta$ was defined and the symmetric and anlisymmelric parts in the shifted energy scale vere found $\left\{D_{\mathrm{A}}(\omega)=\right.$ $1 / 2[D(\omega-\Delta) \quad D(\omega) \Delta)]\}$ The in tegral $A$ of the antisymmetric part was defined as

$$
\left.A \quad\right|_{0} ^{1 \omega} D_{\mathrm{A}}(\omega) d \omega
$$

The shift 1 was varied in steps of $01 \mathrm{mlV}$ until $A_{s}$ was maximized This $\Delta$ was the same for all noble metal point contacts The resulting $D_{\text {, s }}$ are shown in Fig 7 together with literature values for the phonon density of slates oblatned by fit ung Born von Karman models to in elustic neutron icattering datd (14) The close coincidence of the peaks in the sec ond derivalive and the transverse and longitudinal peaks of the phonon struc ture suggest that bulk phonons pldy d

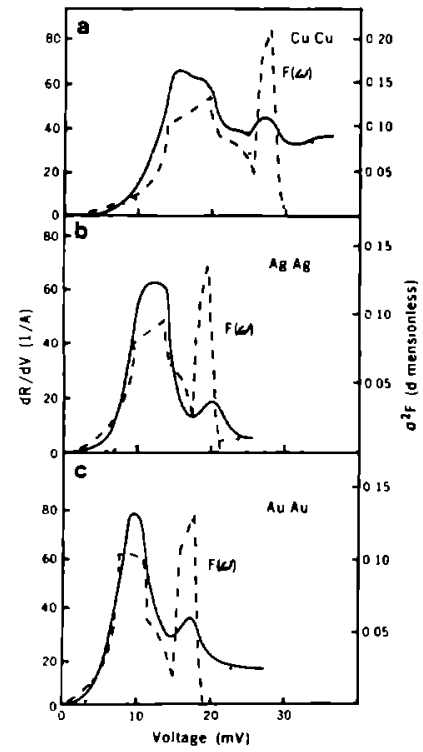


dominant role in the phenomena of point conlacts $A$ s the resistance at zero bids $R_{11}$ is varied the ratio $y$ of the heıght of the first peak to that of the flat portion shove the second peak vanes inveriely with $R_{n}{ }^{2}$ Thus $d R_{1} d V$ contains two ef fects $\alpha^{2} F$ and the effect leading to the relatively fldt $d R$ di ahove the phonon pedks By making point contacl, of ex tremely high $R_{0}$ (> 100 ohms) Yanson (6) has shown that the flat portion can be made small reldtive to the phonon effect

Because of ils relalive smallness at high $R_{n}$ we have called the filat portson the hackground (BG) effecl Yanson only used data with high $R_{0}$ lo find $a^{2} F$ s we wished a more global treatment After some expenmentalion we found that the functional shape

$$
B G(e V)=B\left(\mathrm{dnh}^{2}\left(3 e V / 2 h \theta_{0}\right)\right.
$$

where $k$ is Bolizmann s conslant given in appropriate units and $\theta_{0}$ is the Debye cemperature worked well $B$ is a con stant fit at $30 \mathrm{mV}$ This functional form yields the shorl-dashed curves in Fig 3 The difference hetween $d R / d l$ and the background is the derived absolute $a^{2} f$ from these experıments The nghi-hand scales of Fig 3 are found from the values listed in Table $l$ and the derived relation between $d R / d V$ and $\alpha^{2} F$ Apart from Yanson s values, we know of no other expenmentally determined $a^{2} F^{-4}$ for noble melals in the hterdture To check our results we integrated to find $\lambda$ ds

$$
\lambda=2 \int_{a}^{\alpha} \alpha^{2} F / \omega d \omega
$$

These values are lisied in Table $I$ as $\lambda_{p}$ and are in close (I 15 percent) agreement with the literature values $\lambda_{G}$ (IS) and $\lambda_{4}$ (/6) Using the fitted background function, we could derive similar values

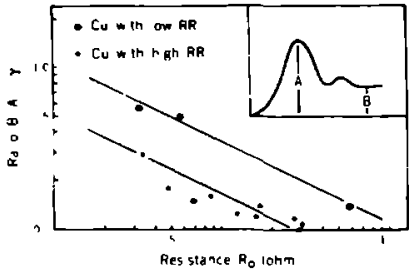

Fig 4 The ratio $B A$ (the background con stant divided by peak height) as d funcion of the resistance $R_{0}$ for two different values of the residual resistivity ratio

of $\lambda$ from $d R / d V$ s for which $R_{0}$ ranged from 2 to $30 \mathrm{ohm}$,

On the basis of the coincidence of the structure of our curves in Fig 3 with the structure of the phonon curves oblaned from neutron scallering data and the good agrecment of our $\lambda$, with those from the litelature we conclude that the voltage derivative of the resistance of tiny point contacts (with background subtracled) provides a simple and convenient tool for medsuring $a^{2} f$ in normal metals

\section{Background Efiect}

We now examine the background ef fect in greater detal In Fig 4 we have plotted the logat ithm of $\gamma$ the ratio of the background constant $B$ to the peak height $A$ of the derived $\alpha^{2} F$. dgainst the logarithm of $\boldsymbol{R}_{\mathbf{0}}$ for poinl contacts formed from copper having (wo different values of the residual resistivity ratıo (RR) The large dots in Fig 4 are for matenal whose RR was 71 The small dots correspond to samples formed from spears of $R R 71$ and anvils of $R R$ more than 4000 . with a geometric mean of 500 Plotted in this way the data shou that the background effect is related to the impunty concentration The constant $B$ varies as RR "With this separation by RR the dald in Fig 4 are well fit by two lines of slope $-1 / 2$ that is $\gamma$ varies ds $\boldsymbol{R}_{0}-12$

We believe that the high current denstltes withe the liny contacts are beneficlal In that electromigration effects will be swift dnv impurity or defeci will be rapidly swept away from the contact In this sense the contacts are self-clean ing References and Motes
$1 \underset{\text { (1973) }}{\text { R S Sorbello } J \text { Phys Chem Solids } 34937}$

$2 \mathrm{~J} C$ Maxwell A Treafrse on Elerincity and Hagnelism (Clarendon Oxford 1904)

$3 \mathrm{H} H$ Holm Electric Contacts (Springer Ver lag Berlin ed 4 1967)

4 A $(, M$ Jansen F $Y$ Mueller P Wyder in Proceedingr of the Second Rochesier Confer ence on Superconductinity in at and f band ther ofs D $\mathrm{H}$ Duuglass Fd (Plenum New York 1976) P 601

5 - Phvs Res B $16 \quad 1329$ (197)

I K Yanson $2 h$ Eksp Tenr Fiz 1035 (1974) in Proceeding of the Intermational Con ference on Low Temperasure Phisics ITIA (North Holland Amsierdam 19rs) paper I I i1 and $\mathrm{Yu} N$ Shalov $7 h$ Eksp Teor $F_{12} 71286(1976)$

$7 T$ Huiben and $K$ de Karl thesis Universily of Nijmegen (1971)

- jM Rowell W I McMillan W I Feldman Phis Res I80 658 ([969)

9 F H Kennard Kinesic Theon of Gases (McGraw Hill New York 1938)

$10 \mathrm{M}$ Knudsen Ann Phis 2075 , 1909 ]

II Yu $V$ Sharvin $7 h$ EAsp Teor Fiz 4998 (19्रs)

$12 \mathrm{M}$ Horn Opuk (Spanger Verlag Berlın 1973)

$13 \mathrm{G}$ Wexler Proc Phis loc Iondon 89927 (1\%6)

14 Copper R M Vicklow G Gilal H G Smith I J Raubenheimer M K Wilkinson Phis Re1 164922 (1967) silver 4 A Kamilaka hara and B M Brockhouse Phi Lefl A 29 M Nicklou Phus Rer B B 4993 (1973)

5 M Nieklow Phvs Rer \& 8 2493 (I97)

16 R F Hoyt and A C Mota Solud Srase Com mun 18139 (1976)

17 This work was performed as part of the research promam of the Suchtung voor Fundamenteel Onderzock der Matene with finaneial suppor from the Nederlandse Organisatie voor Zuiver Wetenschappelujk Onderzoek 
Chapter $\mathrm{V}$ :

JOURVAL DE PHYSIQUE

Colloque C6, supplement au n 8, Tome 39, août 1978, page

C6 602

STRUCTURE OF CURRENT IOLTAGE CHARACTERISTICS OF METAL POINT CONTACTS

A.P. Van Gelder, A.G.M. Jansen, S. Strassler ${ }^{+}$and $P$ Wyder

Research Instztute for Matemals, University of Ihjmegen, Toemoozveld, Nigmegen, The Netherlands

Résumé.- La structure observée dans le $\mathbf{s} 1 \mathrm{gnal}$ de fond de $\mathrm{d}^{2} \mathrm{~J} / \mathrm{dV}^{2}$ est caractérıstıçue des contacts à pointe métalliques. Elle implique la présence d'un processus à deux phonons. On explıque ains l indırectement la forme du signal de fond.

Abstract.- Structure has been observed in the background signal of $\mathrm{d}^{2} \mathrm{~J} / \mathrm{dv}^{2}$ characteristics of metal point contacts, Implying double-phonon backflow-processes. An explanation of the background signal followe indectly from this observation.

Point contacts between metals are useful to determine the parameter $\alpha^{2} F$, the product of the electron-phonon $2 \pi$ teraction strength and the phonon density of states, from the structure of the $\mathrm{d}^{2} \mathrm{~J} / \mathrm{dV}^{2}$ characteristics ( $\mathrm{J}=$ current, $\mathrm{V}=$ voltage) $/ 1-4 /$. Yanson/l/ forms the metal-constriction as a short In a metal-oxyde-metal geometry, whereas our junctions are manufactured by carefully pressing a 'spear' upon an 'anvil' with a differencial screw mechanism in combination with a piezo-electric translator. The spear consists of a metal wire with an electrolytically etched tip ( $0.5 \mu$ ), the anvil 28 a chemically polished metal surface. Our method has permitted an extensive research of high purity materials, like Au/Au (with an RRR $\gtrsim 500$ ). Phase sensitive detection techniques have for instance led to the $d^{2} J / d V^{2}$ characteristic of figure 1 , which exhibits a small reproducible signal if eV > hu, where $\omega_{D} 10$ the Debye Erequency of the metals. In order to explain the observed atructure of the current-voltage characteristica, it 18 mportant to note that the exact geometrical form of the orifice 1 s not a priori known. For this reason, a number $N$ of point contacts (assumed to be identical) 1s considered to represent the real junction, whereas in addition the tunneling probability for the electrons ( $T$ ) may differ from unity. The point contalts are assumed to have a circular orifice with radius $b$. The current $J$ through a point contact has been calculated by solving the semi-classical Boltzmann equation for electrons which interact with the lattice-vibrations and impurities/6/ The coupling between electrons and lattice is characterized by the parameter $\alpha^{2} F=g(\varepsilon)$, a function of the

'Brown Boveri Regearch Centre, CH-540I Baden, Switzerland.

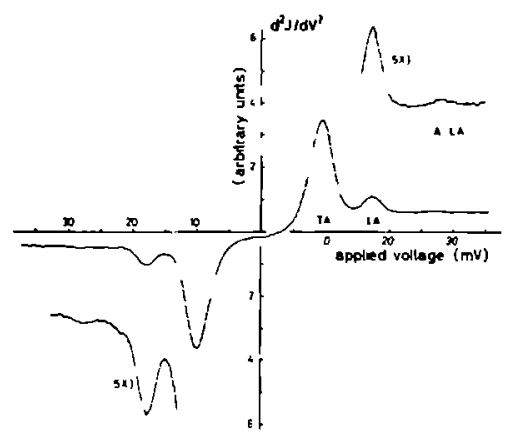

Fig. $1: d^{2} J / d V^{2}$ characteristic (recorder output) of an Au-Au point contact (resistance $R=2.2 \Omega$, temperature of heliumbath $T=1.2 \mathrm{~K}$ ) showing the transverse (TA) and longitudinal (LA) phonon peaks at $10 \mathrm{mV}$ and at $17.5 \mathrm{mV}$. The omall signal at 275 $\mathrm{mV}$ (TA + LA) is believed to be due to double phonon ocatering, followed by a backflow process.

phonon-energy $(\varepsilon)$ such that the energy-dependent electron mean free path $l(E)$ is given by $1(\varepsilon)^{-1}-2 \pi\left(h v_{F}\right)^{-1} \int_{0}^{E} d \varepsilon_{1} g\left(\varepsilon-\varepsilon_{1}\right)$. For our calculatxons/6/ it 18 assumed that the temperature 28 gufficiently low that thermally excited phonons may be Ignored ; this assumption 19 only reasonable for such values of the applied voltage $V$ that eV is large compared with the temperature. If in addition the assumption 13 made that excited phonons may be ignored, the current through the orifice may be expressed as the sum of an (2nfinite) series of terms, which may be labelled according to the number (n) of collisions entering in $2 t$, and supplemented with a factor $b^{n+2}$ in view of dimensional considerations. The leading term, proportional to $b^{2}$, represents the field-emission current through the contact, pro- 
portional to $\mathrm{V}$ and the area of the orifice. The next term, proportional to $b^{3}$, accounts for the backflow of electrons through the orifice. This backflow is possible only after the electron has interacted with the lattice and during the process spontaneously created a phonon. The following term, proportional to $b^{4}$, represents the backflow contribution after two collisions, with a net production of two phonons etc. The kinetic processes carresponding with these terms are shown in figure 2 .

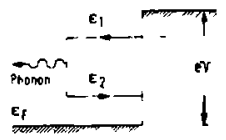

Fig. 2 : Backflou process, following a scattering event where a non-equilibrium phonon is spontaneoug$1 y$ created.

For instance, the single-collision backflow term of the current is proportional to $b^{3} \int_{0}^{e V} d \varepsilon_{2} \int_{\varepsilon_{2}}^{e V} \varepsilon_{1}$ $g\left(\varepsilon_{1}-\varepsilon_{2}\right)$ for the process of figure 2. The experimentally observed structure of $\mathrm{d}^{2} \mathrm{~J} / \mathrm{dV}^{2}$ is hence proportional to $b^{3} g(e V)$, in agreement with reference/1/, but different by a numerical factor of 1.81 . We find that $d^{2} J / d V^{2}=-A T^{2} N b^{3} \cdot g(e V)$, with a constant $A=4 \pi n^{0} e^{3}\left(H v_{F}^{2} m\right)^{-1}$, for this process if $n^{0}, e$ and $m$ are the density, charge and mass of the electrons. The double-collision term gives rise to a contribution to $\mathrm{d}^{2} \mathrm{~J} / \mathrm{dV}^{2}$ proportional to $\mathrm{T}^{2} \mathrm{Nb}^{4} \int_{0}^{\mathrm{eV}} \mathrm{dE} g(\varepsilon)$ $g(e V-\varepsilon)$. The small two-phonon contribution shown in figure 1 is in good agreement with this term, and of order $b / 1(e V)$ in comparison with the single-collision one. It may be concluded that the main background signal saturating to a constant value if eV > hw cannot be explained in terms of multiplescattering backflow processes. The main background signal can be shown to result from stimulated phonon-emission processes, involving the created phonons, and is proportional to $\mathrm{T}^{2} \mathrm{Nb}^{4} \int_{0}^{\mathrm{eV}} \mathrm{d} \varepsilon \mathrm{g}(\varepsilon) /$ $\{\varepsilon \lambda(\varepsilon)\}$, where $\lambda(\varepsilon)$ is the phonon-mean free path. The relatively large magnitude of this term, compared with the double-phonon collision one, results from the smallness of the phonon-mean free path in comparison with $l(E)$. An unambiguous identification of the terms proportional to $b^{3}$ and to $b^{*}$ (background) is possible, and hence of the function $B(e V)$ by comparing the $\mathrm{d}^{2} \mathrm{~J} / \mathrm{dv}^{2}$-characteriatica of different point contacts. Typical values for $\mathbb{N}$ of order
$1 \simeq 5$ have been found for high-ohmic junctions, and tunneling parameters of roughly equal order of magnitude.

\section{References}

/I/ Yanson,I.K., Sov. Phys, JETP 44 (1976) 148 and references therein

/2/ Shalov, Yu.N. and Yanson, I.K., Sov. J. Low Temp. Phys. 3 (1977) 48

13/ Jansen, A.G.M., Mueller,F.M. and Wyder,P., Proc. of the Second Rochester Conference on Superconductivity in d- and f-band Metalo; ed. D.H. Douglass (Plenum Press, New York, 1976)

14/ Jansen,A.G.M., Mueller,F.M. and Wyder,P., Phys, Rev. B 16 (1977) 1325, and Science 199 (1978) 1037

/5/ Lymn,J.W., Smith,H.G. and Nicklow,R.M., Phys. Rev. B 8 (1969) 639

16/ Van Gelder,A.P., Solid State Commun. (in press) 
Chapter VI : POINT CONTACT SPECTROSCOPY IN NORMAL METALS

Reprint Workshop on Current Problems in Superconductivity. Gwatt (Thun), Switzerland, 25-27 october 1979.

A.G.M. Jansen, A.P. van Gelder, F.M. Mueller, S. Străssler ${ }^{X}$ and $P$. Wyder

Research Institute for Materials, University of Nijmegen, Toernooiveld, Nijmegen, The Netherlands

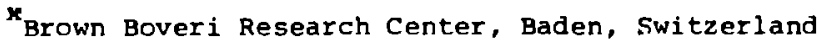

The behaviour of real superconductors can most clearly be described on the basis of the celebrated Fliashberg equations, where the detailed form of the electron-phonon interaction is explicitly taken into account (see Prof. M. Peter, Theory of Real Superconductors). One of the important ingredients of this description is the Eliashberg function $\alpha^{2} F(\omega)$; here, loosely speaking, $a$ is some averaged electron-phonon interaction matrix element, and $F(w)$ is the density of states of phonons with energy $\hbar \omega$. It seems to be rather difficult to get reliahle values for $a^{2} F$, essential for the analysts of superconducting propertles of real materials, from theoretical bandstructure calculations. Therefore it is of considerable importance to be able to measure this function experimentally. In part, this can be done by inelastic neutron scattering or diffuse $X$-ray scattering, where $F(\omega)$ is determined, or with the Mosssbauer effect (measuring the moment $\int a^{2} F(\omega) \omega^{n} \mathrm{~d} \omega, n= \pm 1, \pm 2$ ) or with tunneling spectroscony with superconducting tunnel junctions. As the same electronphonon interaction is responsible for the resistivity, it should also be possible to get $a^{2} F$ from a suitable analysis of the temperature dependence of the electrlcal reststance of metals.

It is the purpose of this note to discuss a new method (point contact spectroscopy) which in principle allows to measure $\alpha^{2} F$ in all reasonably pure normal metals with a not too short mean free path $l$. Point contact spectroscopy has been discovered by Yanson 1,2 , who measured the voltage dependence of the resistance between two evaporated normal metal films, separated by an oxlde layer with a short circult that formed the metallic contact. The same deviations from ohm's law in simple metals can be seen by measuring the voltage dependence of the point contact resistance between an electrochemically sharpened whisker ("spear") (with a curvature radius at the "point" of some $\mu m$ ) pressed against a bulk piece of metal 
("anvil"), single crystals or not ${ }^{3,4}$. Fig. 1 shows directly the recorder output of the current $I$ and its first and second derivative with respect to the voltage as a function of the voltage $v$ applied over the point contact, measured for such a "spear and anvil" arrangement in simple

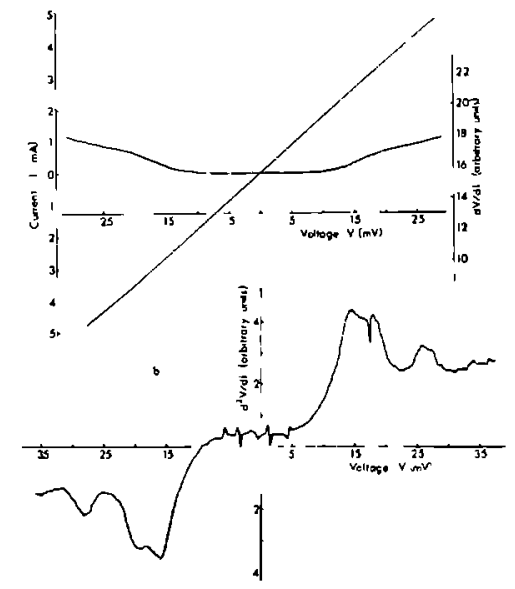

Fig. 1. Recorder sheet with (a) current I and first derivative $\mathrm{dV} / \mathrm{d} \mathrm{I}$, and (b) second derivative $d^{2} v / d I^{2}$ plotted against applied voltage for a $\mathrm{Cu}-\mathrm{Cu}$ point contact. off-the-shelf copper. The structure in the second derivative appears at the tyoical phonon energies of $\mathrm{Cu}$ (one sees clearly two transverse and one longltudinal mode) and it is obvious that this signal is related to the electron-phonon interaction in $\mathrm{Cu}$. Although some exact theories of this effect do exist, based on the solution of the full non-linear Boltzmann equation, we wlll limit ourselves here to a more heuristic and pedagogical discussion.

The problem of the constriction resistance of a small contact between two metals has been studied for the first time by Maxwell quite some time ago $^{5}$. He solved Poisson's equation

for the electrical potential $V$ as a function of the position $\underline{r}, \nabla^{2} V(\underline{r})=0$, in oblate spherical coordinates. Contours of constant potential of a current flowing through a constricting circular orifice of radius a are given by $V(\underline{r})= \pm \frac{V_{0}}{2}[1-(2 / \pi)$ arctan $(1 / \xi)]$, where the oblate spherical coordinate $\xi$ may be found from the more famlliar spherical polar coordinates $r$ and $z$ by solving the implicit equation $r^{2} / a^{2}=\left(1+\xi^{2}\right)\left(1-z^{2} / \xi^{2} a^{2}\right)$ (Fig. 2). The two signs refer to the two sides of the circular orifice. The resistance of such a contact may be found by dividing the total voltage drop (here $v_{0}$ ) by the total current flowing through any contour of constant voltage. For simplicity we choose the contour $V(\underline{r})=0$, that is the orifice itself $(\xi=0)$. The total current is then given by $I=\int d \underline{0} \dot{1}$. Explicitly using Ohm's law in the form $f_{z}=\sigma E_{z}=(1 / \rho) \cdot(\partial v / \partial z)$, where $\sigma=1 / \rho$ is the conductivity of the bulk material, one gets for the Maxwellian resistance

$$
R_{M}=(0 / 2 a)
$$




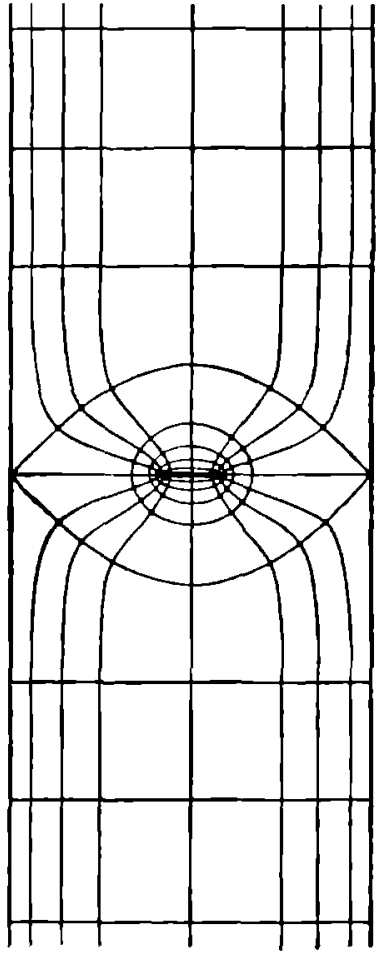

F1g. 2. Maxwell's solution for current- and equipotentiallines for the constriction resistance.

For finite $K$, a suitable Interpolation between

Eqs. (1) and (2) can be

found by solving the

linearized Boltzmann equation with the variational principle, and one gets $^{7}$

$R=(\rho / 2 a) \cdot \Gamma(K)+$

$$
(4 / 3 \pi) \cdot K \cdot(0 / a) \text {. }
$$

It is obvious that the validity of this calculation breaks down if the mean free path $\ell$ of the electrons becomes comparable to the size of the orifice $a, 1 . e$. If the Knudsen number $\mathrm{K}=\ell / \mathbf{a}$ is getting important (Fig. 3) . The limiting case for large $K$ has been discussed by sharvin ${ }^{6}$. If there is a voltage drop $v$ across the contact, the electrons passing through the orifice are getting a speed increment $\Delta v=e v / p_{F}$, where $p_{F}$ is the Fermi momentum. The current due to this speed increment is then given by $I \approx \pi a^{2}$ ne $\Delta v=$ $\pi a^{2}\left(n e^{2} / p_{F}\right) v$, where $n$ is the particle density. Using a Drude-like expression for the resistivity $\rho=p_{F} /\left(n e^{2} l\right)$ one gets for the resistance of a point contact in the high $\mathrm{K}$ regime $\mathbf{R}_{S}=V / I=p l /\left(\pi a^{2}\right)$. An integration over all angles gives the numerical factor in front and leads to the Sharvin resistance

$$
R_{S}=\frac{4}{3} \frac{\rho l}{\pi a^{2}}=\left(\frac{\theta}{3 \pi}\right) K \cdot R_{M} .
$$

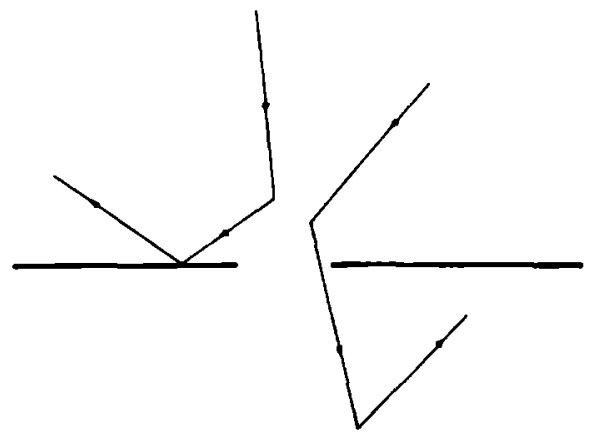

Fig. 3. Point contact in the Krudgen regime. 
$\Gamma(K)$ is a slowly varying function of $K$ with $\Gamma(K=0)=1$ and $\Gamma(K=\infty)=\left(9 \pi^{2} / 128\right)$. The first term in Eq. (3) is like the Maxwell resistance, the second like the Sharvin resistance.

If the mean free path $l$ is energy dependent, $\ell=\ell(E)$, one gets a voltage dependence of $R=R(V)$. As the combination of $p \cdot l$ in the Sharvin-like term in Eq. (3) is independent of the mean free path $l(\varepsilon)$, only the first term enters the partial derivative

$$
\frac{\partial R}{\partial V}=\frac{\partial R_{M}}{\partial V}=\Gamma(K) \frac{\rho l}{2 a} \frac{\partial}{\partial V}\left(\frac{1}{\ell(E=e V)}\right) .
$$

Note that the Sharvin-like term in Eq. (3) leads to no structures; however, the term is essential as he allows to set up an electric field over the point contact, otherwise impossible in a metal.

Assuming the validity of Matthlessen's rule, the total mean free path $\ell$ is given by $\ell^{-1}=\ell_{\text {imp }}^{-1}+\ell_{\text {ep }}^{-1}$, where $\ell_{\text {Imp }}$ is the energy independent scattering length due to impurities. $l_{\text {ep }}$ is the mean free path due to the electron-phonon scattering, $\ell(F)=v_{F} \cdot T(E)$. The lifetime $T(E)$, due to the electron-phonon interaction of an electron with energy $E$ above the Fermi level, is given by using the "golden rule" as

$$
\frac{1}{\tau}=\frac{2 \pi}{\hbar} \sum_{k}\left|g_{q}\right|^{2}\left[\delta\left(\varepsilon_{p}-\varepsilon_{k}-h_{\omega}\right)\left(N_{q}+1-f_{k}\right)+\delta\left(\varepsilon_{p}+h \omega_{q}-\varepsilon_{k}\right)\left(N_{q}+\varepsilon_{k}\right)\right] .
$$

Here, $g_{q}$ is the matrix-element for the electron-phonon Interaction for a phonon of momentum $q$ and energy $h_{\omega_{q}} \varepsilon_{p}$ and $\varepsilon_{k}$ the energies of electrons with momenta $p$ and $k, N_{q}$ the Bose distribution function of the phonons and $f_{k}$ the Fermi distribution function of the electrons. Replacing the k-summation in the usual way

$$
\sum_{k}=N_{0} \int d \omega \int \frac{q d q}{\left(2 k_{F}^{2}\right)}
$$

where $N_{0}$ is the density of states at the Fermi level, and introducing the Eliashberg function

$$
a^{2} F(\omega)=N_{0} \int\left(\frac{q d a}{2 k_{F}^{2}}\right)\left|g_{q}\right|^{2} \delta\left(\omega-\omega_{q}\right)
$$

one gets

$$
\frac{1}{\tau(E)}=\frac{2 \pi}{h} \int d \omega \alpha^{2} F(\omega)[2 N(\omega)+1-f(E-h \omega)+f(E+h \omega)] .
$$


At $T=0$, this reduces to

$$
\frac{1}{\tau(E, T=0)}=\frac{2 \pi}{\hbar} \int d \omega a^{2} F(\omega) \theta(h \omega-F) .
$$

Therefore, one finally gets as the grand result for the voltage derivative of the point contact resistance

$$
\frac{d R}{d V}(V)=\frac{\rho \ell}{2 a} \Gamma(K) \frac{2 \pi e}{\hbar v_{F}} a^{2} F(e V)
$$

Eq. (10) allows a direct determination of the energy dependence of the Eliashberg function $a^{2} F(w)$.

Fig. 4 shows the point contact spectrum $d R / d V$ of $A u$, together with $F(w)$ determined by inelastic neutron scattering. As expected, the van Hove singularities measured by the two different experiments coincide. Fig. 5 shows point contact spectra for all noble metals, again together with $F(\omega)$

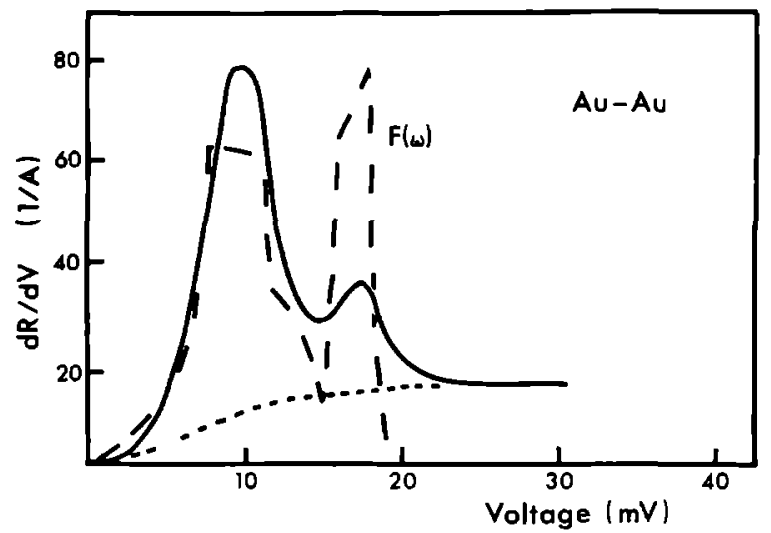

Fiq. 4. Point contact spectrum of a Au-Au point contact. Long-dashed curve: Phonon density of states from inelastic neutron scattering. Short-dashed curve: Background signal.

as determined by neutron scattering. As an interesting detail, Fig. 5d shows the point contact spectrum of a Au-spear with a Cu-anvil; the phonons of both metals. $\mathrm{Au}$ and $\mathrm{Cu}$, can clearly be detected indicating that spear and anvil together are responsible for the signal. From the point of view of understanding the electron-phonon interaction in noble metals, it is very important to note that the ratio's of the $a^{2} F$ to the related $F(\omega)$ 's show a strong $\omega$ or energy dependence. Transverse phonons couple to the electron gas about four times more effectively 


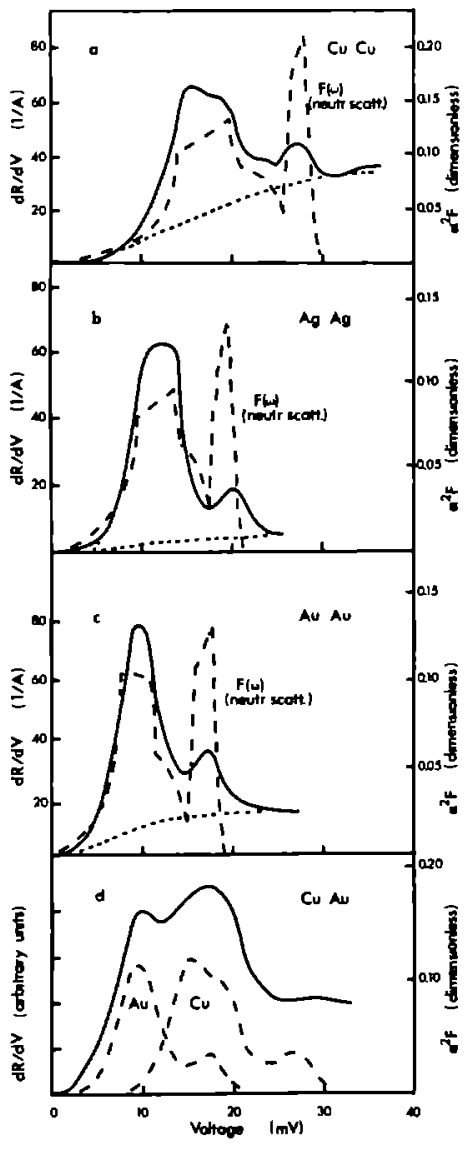

Fig. 5. Measured voltage derivative of the resistance of point contacts as a function of applied voltage of (a) $\mathrm{Cu}-\mathrm{Cu}$, (b) Ag-Aa, (c) $\mathrm{Au}-\mathrm{Au}$ and (d) Cu-Au plotted as the solid lines. The right hand scale is the derived value for $\alpha^{2} F_{p c}$. In (a), (b) and (c), the background functions are shown as short-dashed curves and the phonon density of states from neutron scattering as long-dashed curves.

than longitudinal phonons in noble metals. This is consistent with the results of bandstructure calculations showing that d-electrons are important and are strongly umklapp coupled to transverse phonons in noble metals. It is interesting to compare $\alpha^{2} F$ as measured with point contact spectroscopy with theoretical bandstructure calculations of the electron-phonon interaction. The most detailed and reliable pseudopotential calculations have been carried out on the alkal1s 8 . Figs. 6, 7 and 8 show point contact spectra for $a^{2} F$ for $L i$, Na and $K$, together with theoretical calculations, and $F(\omega)$ determined from inelastic neutron scattering. The agree-

ment is remarkable and conflrms the pseudopotential calculations. Note that in $\mathrm{Li}$, just as in the case of the noble metals, the electrons are more strongly coupled to the transverse phonons than to the longitudinal ones, while in the case of $\mathrm{Na}$ and $\mathrm{K}$ it is the other way around.

It is very clear and obvious that the theoretical discussion on the mechanism of point contact spectroscopy as sketched here is at most a guldeline for experiments. The full nonlinear Boltzmann equation has to be solved in order to understand these highly nonlinear phenomena. This sort of solutions are now becoming avallable 9,10 . Most of them are based on the "back-scattering" concept of van Gelder ${ }^{10}$ : The solutions of the Boltzmann equation are expanded in terms of the collision kernel. The zeroth approximation is a Sharvin-like field-emission term where electrons 

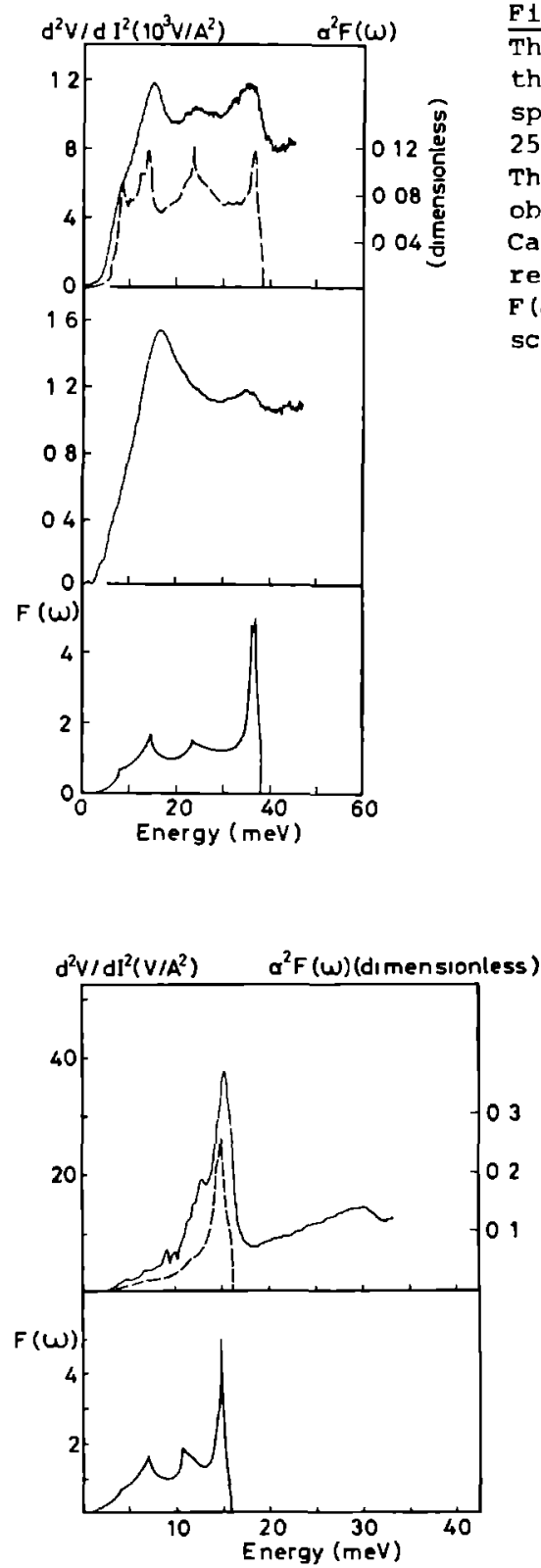

Fig. 7. Point contact spectroscopy of $\mathrm{Na}$ (junction resistance $2.9 \Omega$ ). The dashed curve ( $r$ ight hand scale) is the theoretically ohtained function $a^{2} \mathbf{F}(\omega)$ (Carbotte and Dynes). The curve in the bottom part represents the phonon density of states $F(\omega)$ obtained from inelastic neutron scattering experiments.
Fig. 6. Polnt contact spectroscopy of Li. The two solid curves (left hand scale) in the top part are measured point contact spectra for $\mathrm{Lj}$ (junction resistances $25.4 \Omega$ (uppermost curve) and $11.8 \Omega$ ). The dashed curve is the theoretically obtained function $a^{2} F(w)$ (Hayman and Carbotte). The curve in the bottom part represents the phonon density of states $F(\omega)$ obtalned from inelastic neutron scattering experiments.

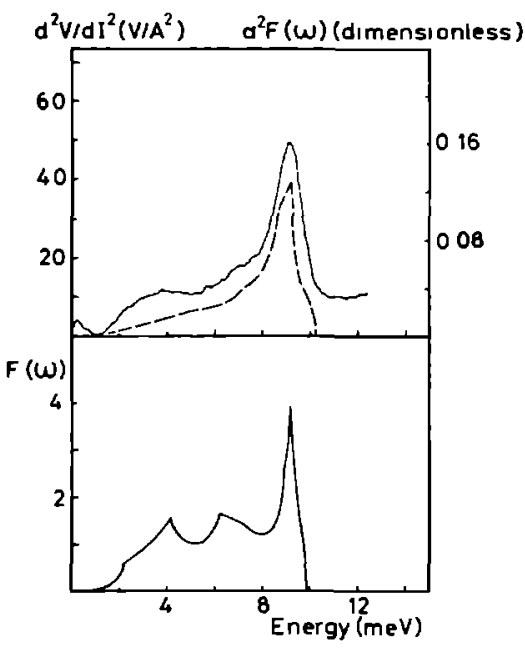

Fig. 8. Point contact spectroscopy of $\mathrm{K}$ (Junction resistance $1.1 \Omega$ ). 
are injected into the other metal, leading to the disturbed Fermi surfaces as sketched in Fig. 9. While this does not lead to a voltage dependent structure in the signal, the first order term is given by the back-streaming of the electrons through the orifice, after one scattering with a phonon. This term leads to a decrease in the total net-current and therefore to an increase in the reslstance, an essential difference with the usual tunneling process through an oxide layer which should be pointed out clearly. The second order corrections ${ }^{11}$ include collisions with two phonons before the electron is scattered back through the orifice; this leads to a signal at twice the characteristic phonon energy, and can clearly be seen in Fig. 7 at energies of around $30 \mathrm{meV}$, while the typical Debye energy is at $15 \mathrm{meV}$.

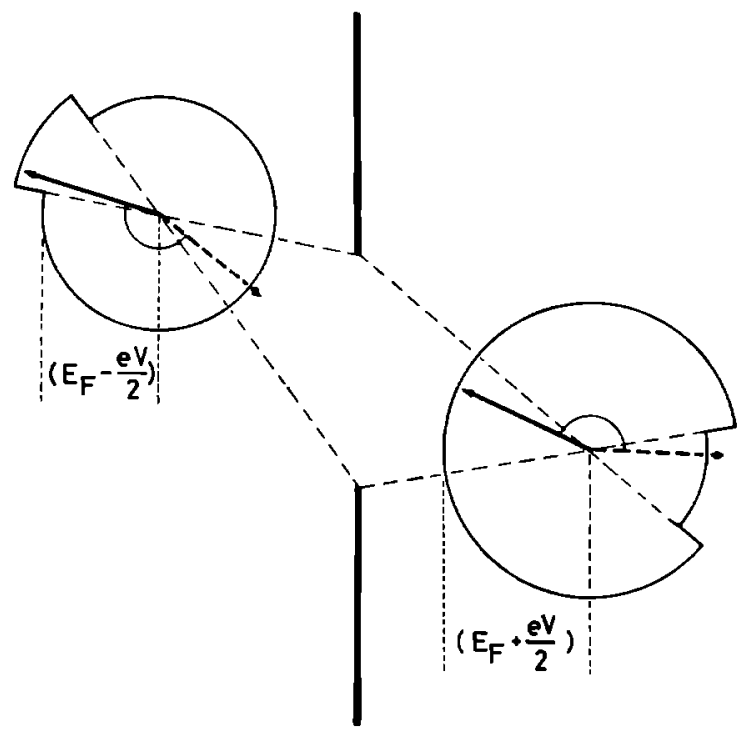

Fig. 9. Zeroth approximation electronic distribution function for the theoretical analysis of point contact spectroscopy.

In addition, due to the kinematics involved, the theory predicts an interesting efficiency factor. The measured signal is not quite $a^{2} F$, but

$$
\left.S(w)=N_{0} \int \frac{q d q}{2 k_{F}^{2}}\right)\left|g_{q}\right|^{2} \delta\left(w-w_{q}\right) n(q)
$$

where the efficiency factor $n(q)$ is a function of the scattering angle $\theta=\theta(\mathbb{D}, \underline{k})$. For instance in the expression for the simple dc-resistivity $\rho$, 
$n$ leads to the well known transport-relaxation time with the famous $n_{d c}(q)=\{1-\cos \theta(\underline{p}, \underline{k})\}$, while in the case of poirt contacts one has

$$
\eta_{p c}=\frac{1}{2}\{1-\theta / \operatorname{ta} \theta\} .
$$

As follows from $\mathrm{Eg}$. (12), $\eta_{p c}$ is effective for low phonon energies. This can clearly be seen in Fig. 10 where $a^{2} F$ measurements from point contact spectroscopy in the normal state of $\mathrm{Pb}$ are compared with measurements of $a^{2} F_{t}$ from strong coupling tunneling in the superconducting state using the Rowell-McMlllan scheme.

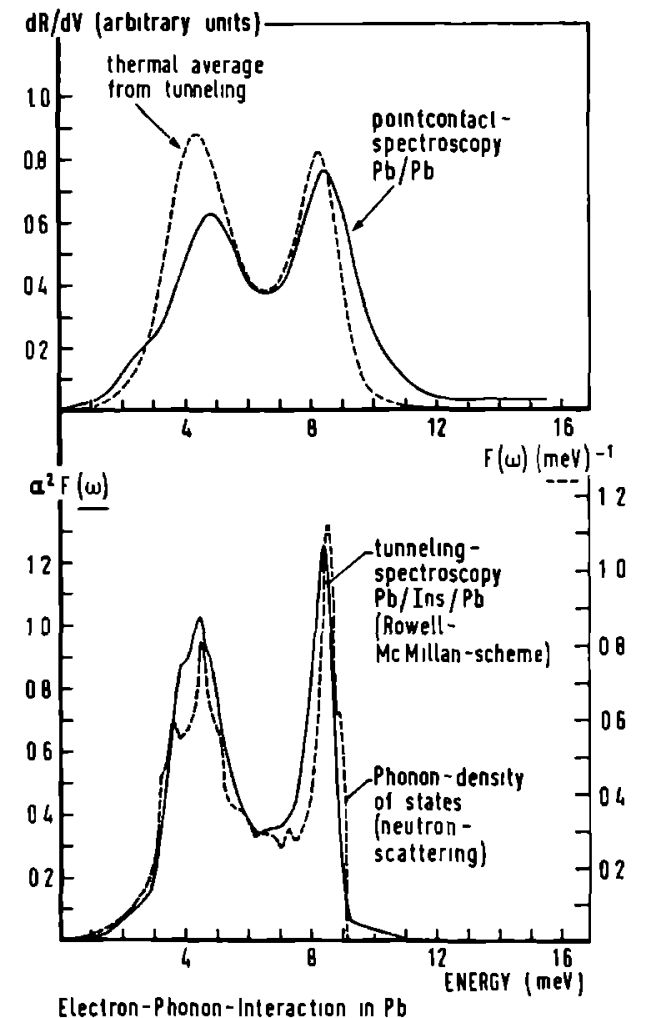

Fig. 10. Comparison of $\mathrm{a}^{2} \mathrm{~F}$ of $\mathrm{Pb}$ as measured by point contact spectroscopy in the normal state and superconducting tunneling in the superconducting state.
As there are no phonons present above the Debye cutoff frequency $\omega_{D}, 1 . e$. $F\left(\omega>\omega_{D}\right)=0$, the point contact spectrum should vanish for energles ev $>h_{\omega_{D}}$. Nevertheless, as is indicated in Fig. 2 with the short-dashed curve, a "background signal" is present on top of the $a^{2} F_{p c}$-signal which levels off at a constant non-zero value for energies above $\omega_{D}$. This can be understood by realizing that in these highly nonlinear processes the presence of phonons which are not in thermal equilibrium has to be taken expl1citly into account. It is possible to show that this background signal (constant for energies above $\omega_{D}$ ) is due to the stimulated enission of phonons during the back-flow process. 
1. I.K. Yanson, Zh. Eksp. Teor. F1z. 66, 1035 (1974)

[Sov. Phys. - JETP 39, 506 (1974)].

2. I.K. Yanson and I.O. Kulık, J. Phys. (Par1s) 39, C6-1564 (1978), and references therein.

3. A.G.M. Jansen, F.M. Mueller and P. Wyder, Phys. Rev. B16, 1325 (1977).

4. A.G.M. Jansen, F.M. Mueller and P. Wyder, Sclence 199, 1037 (1978).

5. J.C. Maxwell, A Treatise on Electricity and Maqnetism (Clarendon, Oxford, 1904).

6. Yu,v. Sharvin, Zh. Eksp. Teor. F1z. 4 B, 984 (1965)

[Sov. Phys. - JETP 21, 655 (1965)].

7. G. Wexler, Proc. Phys. Soc. Lond. 89, 927 (1966).

8. B. Hayman and J.P. Carbotte, J. Phys. F: Metal Phys. 1, 828 (1971); J.P. Carbotte and R.C. Dynes, Phys. Rev. 172, 476 (1968).

9. I.O. Kul2k, A.N. Omel'yanchuk and R.I. Shekheter, F1z. N1zk. Temp. 3. 1543 (1977) [Sov. J. Low Temp. Phys. 3, 740 (1978)] .

10. A.P. van Gelder, Solid state Commun. 25, 1097 (1978).

11. A.P. van Gelder, A.G.M. Jansen, S. Străssler and P. Wyder, J. Phys. (Par1s) 39, C6-602 (1978). 
Chapter VII : TEMPERATURE DEPENDENCE OF POINT-CONTACT SPECTROSCOPY IN COPPER.

\section{ABSTRACT}

The current-voltage characteristics of $\mathrm{Cu}$ point contacts have been studied experimentall $Y$ between 5 and $300 \mathrm{~K}$. The effect of the temperature on the measured $a^{2} v / d I^{2}$-curves is a broadening of the spectrum for the electron-phonon interaction in the metal. The measured resistance of a point contact is compared with the resistivity of bulk material as a function of the temperature. An analysis of the broadening of the spectra and the temperature dependence of the contact resistance is given. Differences between the temperature dependences of the resistance of a point contact and that of the bulk are ascribed to different transport efficiencies for the electron-phonon interaction in these two cases. 
I. Introduction.

During the past few years a new spectroscopıc method has been employed for studying the electron-phonon interaction in metals using point contacts between metals. The experimentally observed current (I) - voltage (V) characteristics of a metaliıc contact at liquid helium temperatures are non-Iınear and allow a direct determination of the well known Elıashberg function $\alpha^{2} F(\omega)$ by measuring the second derivative $d^{2} v / d I^{2}$ as a function of the applied voltage ${ }^{1,2} \cdot \alpha^{2} F(\omega)$ is the product of the density of states of the phonons and the squared matrix element for the electron-phonon interaction, averaged over the Fermi surface. The great importance of this point-contact method lies in the fact that it is up to now the only existing method to obtain such detalled information about the electronphonon interaction in normal metals. Recently also theoretical work 3,4 becomes avallable, which solves the Boltzmann equation for the pointcontact problem in order to explain the observed phenomena in the experiments.

In this paper we present point-contact experiments, which for the first time have been performed within the entire temperature range between Iıquia helıum and room temperature, in order to investıgate the temperature dependence of the spectroscopic data. With increasing temperature the experiments show that the measured $a^{2} \mathrm{~V} / \mathrm{dI}^{2}$-spectrum is broadened due to thermal averaging. A weight function given from a theoretical analysis of the point-contact problen describes the broadening very well. Furthermore, the theory predicts an analogous behaviour for the temperature dependence of the point-contact resistance and that of the bulk resistivity arising from the electron-phonon interaction. Our experiments show a strong similarity between the measured point-contact resistance and the 
bulk resistivity as a function of temperature. Small differences between the point-contact resistance and the bulk resistivity may be ascribed to small differences of the efficiency of the electron-phonon scattering process. In the next section we will discuss theoretically the current through a metallic point contact as a function of the temperature and the applied voltage. From the expression for the current we obtain the voltage derıvatıves (first and second) of the current. The experiment will then be described, and the experimentally determined $d v / d I-$ and $d^{2} v / d I^{2}-$ curves for $\mathrm{Cu}$ point contacts are presented and analysed theoretically.

II. Current-voltage relations for a point contact.

The interesting behaviour of a point contact in the clean limit, $1 . e$. If the linear dimension a of the metallic contact is smaller than the mean free path $\ell$ of the electrons, has been realized for the first time by Sharvin ${ }^{5}$. Due to the applied voltage over this type of junction, an electric field exists within the metal in the region of the contact, and the electrons are accelerated by the electric field near the orıfice. Via Inelastic scattering processes with elementary excltations (e.g. phonons) in the metal a relaxation of the accelerated electrons takes place, ylelding a non-linear current-voltage characteristic as a result of the energy-dependent electron-phonon interaction. To calculate the current through the orifice one reeds the distribution function of the electrons. The Boltzmann equation for the distribution of the electrons has been solved for this problem by iteration with respect to the collision term, taking into account the appropriate boundary conditions ${ }^{3,4}$.

In zeroth order (where the collision term in the Boltzmann equation is zero) a distribution function is obtained for the electrons with an 
energy difference for electrons which have or have not passed through the orifice, due to the potential drop ev over the contact. Using this disturbed distribution function, the sharvin current ${ }^{5}$ is obtained and is given for a circular orifice with ridius a by

$$
I_{0}=\frac{3 \pi a^{2}}{4 e \rho l} \int_{-\infty}^{\infty} d \varepsilon[f(\varepsilon-e V)-f(\varepsilon)]=\frac{3 \pi a^{2}}{4 \rho l} v=\frac{V}{R_{0}}
$$

The product $\rho \ell$ ( $\rho$ is the resistivity and $\ell$ the mean free path of the electrons) is a materıal constant and satısfies $\rho \ell=m v_{F} / n_{0} e^{2}$ (Drude formula), where $m$, $e$ and $v_{F}$ are the mass, the charge and the Fermi velocity of an electron and $n_{0}$ the density of electrons in the metal. $f(E)$ is the equilibrium Fermi distribution function for electrons with an energy $\varepsilon$ with respect to the Fermi level. We havo assumed that the electronic density of states is constant near the Fermi energy $E_{F}$ for applied voltages with $e V \ll E_{F} ;$ self-energy effects are hence ignored.

In next order, single collisıons involving phonons are taken into account. These inelastıc collısıons give a negative correction to the current, because the electrons can change their momentum and flow back through the orlfice after a scattering process has occurrcd. In first order, considering only single collisions and ignoring departures from equilibrium of the phonon system, the correction to the current is given by

$$
\begin{aligned}
I_{1}=-\frac{4 \pi a^{3}}{h e v_{F} \rho l} & \int_{-\infty}^{\infty} d \varepsilon[f(\varepsilon-e V)-f(\varepsilon)] \\
& \times \int_{0}^{\infty} d \omega \alpha^{2} F_{p}(\omega)[1+2 v(\omega)+f(\varepsilon+\omega)-f(\varepsilon-\omega)],
\end{aligned}
$$


where $v(w)$ is the Bose-Einstein distribution for the phonons. The factor $[f(\varepsilon-e V)-f(\varepsilon)]$ equals the density of injected electrons of energy $\varepsilon$, relative to the Ferml level of the low-voltage side of the contact. Tne energy levels $\varepsilon+\omega$ (with respect to the low voltage side) correspond with electrons, which flow back through the orlfice after spontaneous or stimulated emission $(\varepsilon-w)$, or stimulated absorption $(\varepsilon+w)$ of a phonon with energy w has taken place. In the expression above the electron-phonon interaction function $\alpha^{2} F$ has a subscript $p$ to indicate that in the specific point-contact geometry $\alpha^{2} F_{p}$ alffers from the usual $\alpha^{2} F$ of Ellashberg by an efficiency function $\eta(\theta)=\frac{1}{2}(1-\theta / \operatorname{tg} \theta)$, whlch depends on the scattering angle $\theta$ between the velocitydirections of the electron before and after the collision event with a phonon ${ }^{6}$. In a similar way, one has defined in the DC-transport theory for the calculation of the electrical resistivity the function $\alpha^{2} F_{t r}{ }^{7}$, lnvolving the well-known efficiency function $(1-\cos \theta)$. If the temperature $T$ is zero, equation (2) reduces to 4

$$
I_{1}=-\frac{4 r a^{3}}{h e v_{F} \rho \hat{\chi}} \int_{0}^{e V} d E_{2} \int_{0}^{\varepsilon_{1}} d \varepsilon_{1} \alpha^{2} F_{p}\left(E_{1}\right)
$$

It should be noted that $I_{1}$ is of first order $1 \mathrm{n} \mathrm{a} / \mathrm{l}_{\mathrm{ep}}$, because $I_{1} \sim I_{0} a / l_{e p} ;$ here

$$
1 / \ell_{\text {ep }}(\varepsilon)=\frac{2 \pi}{h v_{F}} \int_{0}^{\infty} d \omega \alpha^{2} F(\omega)[1+2 \nu(\omega)+f(\varepsilon+\omega)-f(\varepsilon-\omega)]
$$

is the inverse of the electron-phonon scattering length for an electron with energy $\varepsilon$ above the Fermi level (Fermi's golden rule). 
In point-contact spectroscopy, the derivatives of the current-voltage characteristics show interesting phenomena. Differentiating the current $I=I_{0}+I_{1}$ with respect to the applied voltage the temperature dependence of the resistance at zero voltage is given by:

$$
R_{0}(T)=R_{0}+R_{0}^{2} \frac{4 \pi a^{3}}{h v_{F} \rho l} \int_{0}^{\infty} d \omega \alpha^{2} F_{p}(\omega) \frac{\omega / 2 k_{B} T}{\sinh ^{2}\left(\omega / 2 k_{B} T\right)}
$$

for the case where $R_{0}(T)-R_{0}<R_{0}$. A similar expression can be found for the temperature dependent resistivity in bulk material with the electronphonon interaction function $\alpha^{2} F_{\operatorname{tr}}{ }^{7}$ :

$$
\rho(T)=\frac{2 \pi m}{h_{n_{0}} e^{2}} \int_{0}^{\infty} d \omega \frac{\omega / 2 k_{B} T}{\sinh ^{2}\left(\omega / 2 k_{B} T\right)} \alpha^{2} F_{t r}(\omega) .
$$

The second derivative of the current $I=I_{0}+I_{1}$ with respect to the voltage $V$ is

$$
\frac{d^{2} I}{d V^{2}}=-\frac{4 \pi a^{3} e}{h v_{F} \rho l} \int_{-\infty}^{\infty} d \omega \alpha^{2} F_{P}(\omega) \times\left(\frac{\omega-e V}{k_{B} T}\right)
$$

$x(z)$ is a bell-shaped function with half-width $5.4 \mathrm{k}_{\mathrm{B}} \mathrm{T}$ :

$$
x(z)=\frac{1}{k_{B}^{T}} e^{z} \frac{(z-2) e^{z}+z+2}{\left(e^{z}-1\right)^{3}}
$$


In inelastıc tunneling spectroscopy for metal-oxide-metal structures with normal metal films a similar thermal broadening was found ${ }^{8}$. For $T=0$ $\mathrm{d}^{2} \mathrm{I} / \mathrm{dV}^{2}$ becomes proportional to $\alpha^{2} \mathrm{~F}_{\mathrm{p}}(\mathrm{eV})$ and in point-contact experiments at liquid helium temperatures a direct determination of the electron-phonon interaction is possible. We have measured the spectra $d^{2} V / d I^{2}\left(=-R^{3} d^{2} I / d V^{2}\right)$ for $\mathrm{Cu}$ point contacts between low temperatures and roomtemperature. Using the spectrum for the lowest measuring temperature (5 K), which is proportional to $\alpha^{2} F_{p}$, it is possible to check the validity of equation (7). In addition, we have also investigated the temperature dependence of the point-contact resistance at zero voltage and compared this result with the measured electrical resistivity of bulk material.

\section{Experiments and results.}

The experiments were performed for copper point contacts. The point contacts consisted of a sharply etched needle, pressed against a flat surface of bulk material. The needle was fabricated by electro-chemically etching of a polycrystalline cu wire, $50 \mu$ in dianeter. The other part of the contact was a single crystal of $\mathrm{Cu}$ with the (110)-plane parallel to the contact surface. The point contact was mounted in a holder, which was totally fabricated from copper except for the electrical isolation, to avoid the instability of the contacts upon cooling down because of different thermal expansion coefficients of the materials used in the sample holder. With a screw mechanism stable metallic contacts were adjusted at roomtemperature and mounted in a variable temperature cryostat to control the temperature between 300 and $5 \mathrm{~K}$ with an accuracy of $0.5 \mathrm{~K}$. In this configuration it was possible to obtain stable point contacts, and reliable in the sense that the contact geometry remained unchanged during a run 
between roontemperature and helıumtemperature. The derıvatıves ( $\mathrm{dV} / \mathrm{dI}$ and $d^{2} v / d I^{2}$ ) of the current-voltage characterıstics were measured using phase sensitive detection with modulation voltages in the range of $500 \mu \mathrm{V}$. Because the change in the contact resistance is only a few percent, we used a bridge circull ${ }^{9}$ to compensate the unchanging part of the resistance in order to achıeve higher resolution.

In the Figs. 1 and 2 we have plotted the measured $d^{2} v / d I^{2}$-curves for $\mathrm{Cu}-\mathrm{Cu}$ point contacts between 5 and 40 resp. 270 ( $\mathrm{K})$. The observed second derivative is an anti-symmetric function around zero voltage. For the lowest measuring temperature, the transverse (17 mV) and longltudinal (28 mV) phononpeaks are clearly visıble. The measured structure in the Cu point-contact spectra is characterıstic for point-contact spectroscopy and has been observed in pressure-type point-contact experiments in all tre noble metals ( $\mathrm{Cu}, \mathrm{Ag}$ and $\mathrm{Au}$ ) at lıquid helium temperatures ${ }^{2}$. According to formula (7), the measured spectrum at low temperatures will be proportional to the electron-phonon interaction $\alpha^{2} F_{p}(e V)$. Sometirnes we observed (see Fig. 2) structure at the sum-frequency of transverse and longitudinal phonons $(45 \mathrm{mV})$. These signals at double phonon frequencies were recently observed in point-contact experiments ${ }^{10}$ and can be explained by an extra iteration in the solution of the Boltzmann equation involving double collısion processes ${ }^{4}$. For voltages higher than the Debye energy, the measured signal should vanısh because the phonon density of states is $z \epsilon$ ro. In the experiment however, a smooth background signal is observed which is nearly a constant above the Debye energy. It has been realized that the phonons are not in thermal equilibrium at the orifice ${ }^{10}$. The non-equilibrium phonons act as additional scattercenters for the electrons (stimulated emission and absorption processes), which yield an extra negative term to the current. By solving the transport equation for the 


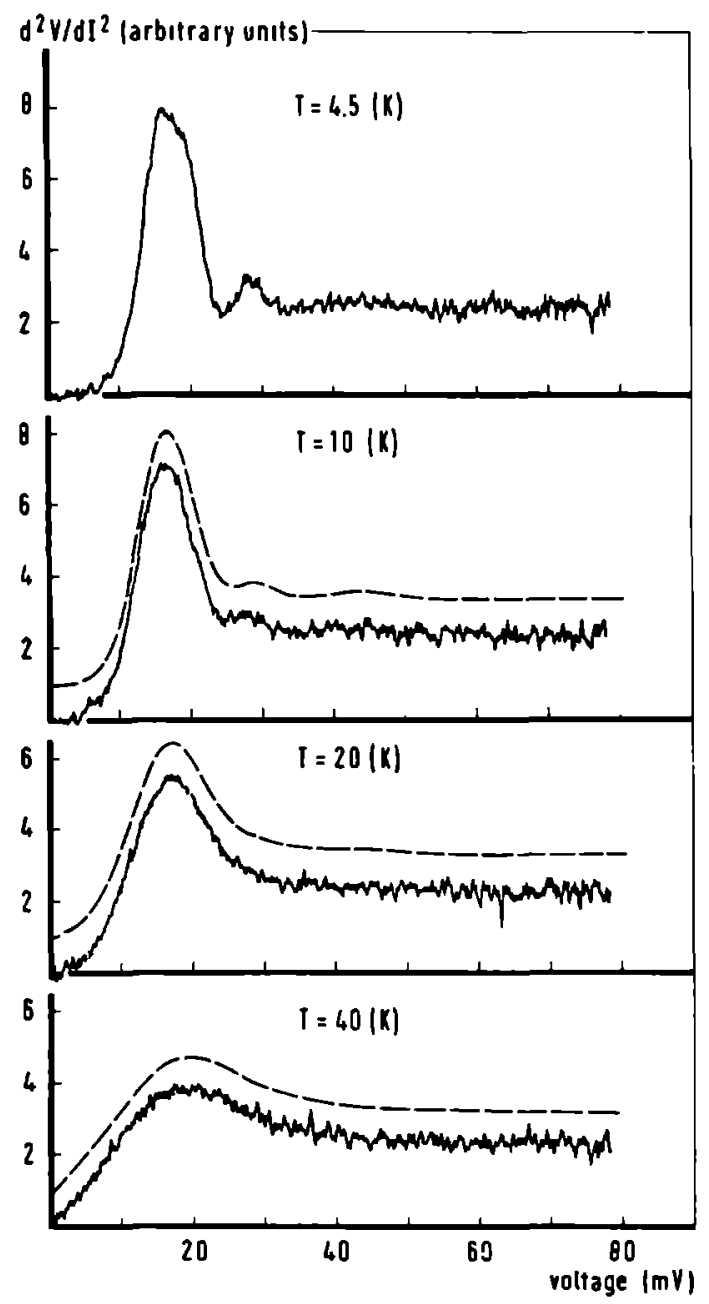

Fig. 1 : Point-contact spectra for Cu between 4.5 and $40 \mathrm{~K}$. The dashed lines (shifted one division on the vertical scale) have been calculated by means of the theoretical given thermal average of the spectrum at $4.5 \mathrm{~K}$. The point-contact resistance was $2.2 \Omega$ at low temperatures. 


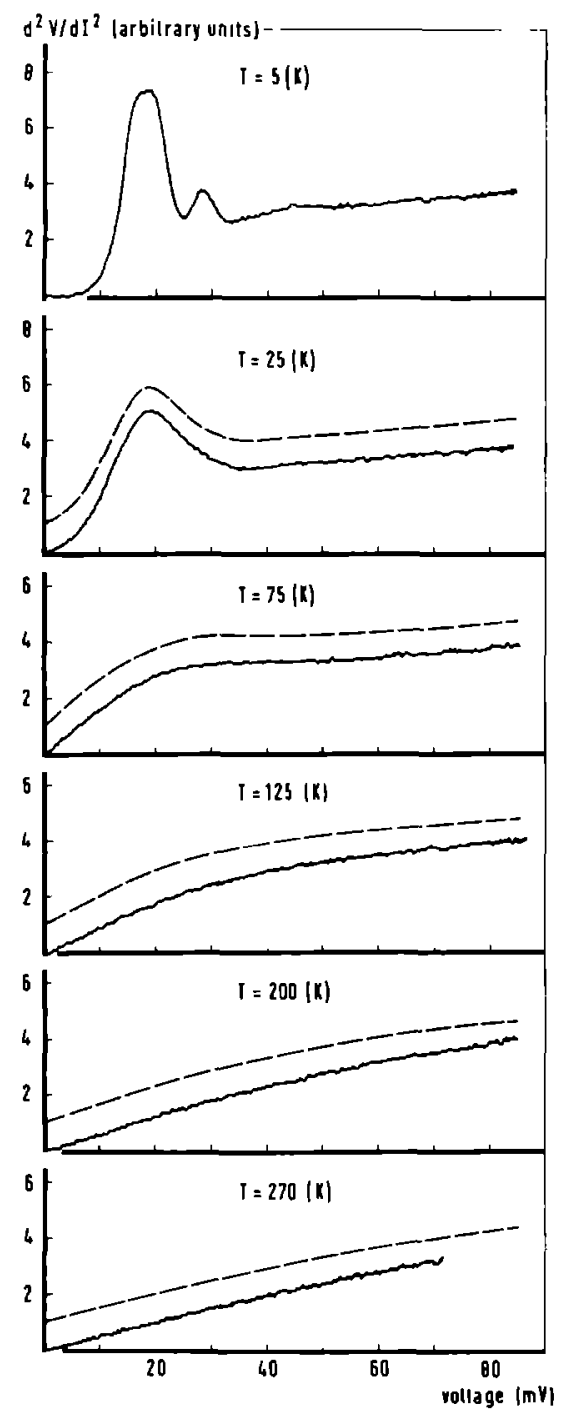

Fig. 2 : Point-contact spectra for $\mathrm{Cu}$ between 5 and $270 \mathrm{k}$. The dashed lines (shifted one division on the vertical scale) have been calculated by means of a theoratical given thermal average of the spectrum at $5 \mathrm{k}$. The polnt-contact resistance was $1.7 \Omega$ at low temperatures. 
phonons a signal in the point-corlact spectrum has been found, which is constant at voltages above the Debye energy 10 .

By increasing the tenperature from heliumterperature up to roomtemperature, the point-contact spectra were smeared out due to thermal averaging. Eq. (8) gives an expression for the thormal averaging function $x$. Using the measured spectrum at $\mathrm{T}_{0}-\sim 5 \mathrm{~K}$, we have calculated by a convolution product the spectra at higher iemperatures $T$ as follows:

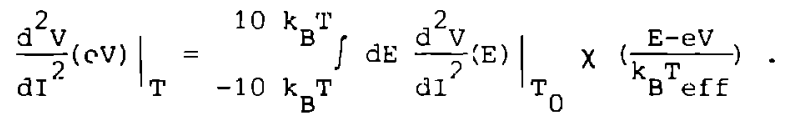

In the argument of the function $x$, we have taken an effective temperature $\mathrm{T}_{\text {eff }}=\sqrt{\mathrm{T}^{2}-\mathrm{T}_{0}^{2}}$ unstead of $\mathrm{T}$, as we are int.crested in the spcctral averaging due to the temperature difference between $\mathrm{T}$ and $\mathrm{T}_{0}$. We have subtracted the temperature $T$ from $T_{0}$ quadratically in analogy to the guadratical addition of the standard deviations, or equivalently the linewidths for tne superposition of Gadssian distributions. The introduction of this effective temperature will only have an effect on the andysis of the spectra at the lowest measuring temperatures. For the signal values at voltages beyond the measured voltage range, a linear interpolation of the spectrun at the lowest medsuring temperature was used. The calculated results, indicated by the dashed 1 ines in the Figs. 1 and 2 , show that the thermal averaging is very well described by the function $x$. In section II we haven't discussed the influence of the temperature on the background function, on which the electron-phonor interaction function $\alpha^{2} F_{p}$ is superimposed. Because the background function will be a smooth function of the applied voltage, the averaging will nardly infiuince the 
background for temperatures $\mathrm{T}<100 \mathrm{~K}$. For temperatures $\mathrm{T}>100 \mathrm{~K}$ the experiments show that the broadening of the background is equally well described by the function $x$.

A great part of the temperature dependence of the resistivity in a metal is determined by the electron-phonon interaction. Eqs. (5) and (6) show that the behaviour as a function of temperature is similar for the resistance of a point contact and the resistivity of a bulk metal, except for differences in the efficiency of the collisions. To look into this problem, we have also measured in detall the resistance of a point contact at zero voltage as a function of temperature. First we will look at the qualitative difference in the contact resistance between roomtemperature and liquid heliumtemperature. It is not unreasonable that the weighted integrals over $\alpha^{2} F_{p}$ and $\alpha^{2} F_{t r}$ in Eqs. (5) and (6) are roughly equal, because in the high temperature limit the weighted integrals reduce to the expression for the mass-enhancement parameter $\lambda=2 \int \alpha^{2} F(\omega) / \omega$ d $\omega$ for the electron-phonon interaction, which can be shown to be equal for $\alpha^{2} F_{p}$ and $\alpha^{2} F_{t r}$ due to a sum-rule argument ${ }^{6}$. With the equality for the weighted integrals we obtain for the temperature dependent part of the resistance of a metallıc contact, usıng Eqs. (1), (5), (6) and the Drude formula $\rho \ell=m v_{F} / n_{0} e^{2}:$

$$
R(T)-R_{0}=0.360 \frac{p(T)}{a} \text {. }
$$

This expression resembles the Maxwellıan constriction-resistance ${ }^{11}$ $\left(R_{M}=\rho / 2 a\right)$ for a circular contact in the dirty limit, 1.e. the electronic mean free path is small compared to the linear dimension of the contact, where Ohm's law is valıd. Using formula (10) and the resistivity $\rho(T)$ at 
roomterperature we can estimate from the experimentally measured variation in resistance between helium- and roomtemperature the linear dimension d of the orlfice. The radius of the orifice can also be determined from tine Sharvin resıstance measured at low temperatures (Eq. (1)). Using Eqs. (1) and (10) we established the values of the radius of the contact for all measured point contacts, which agreed within 10 : for the two determinations. From this we can conclude that the assumption of a single circular orifice gives a realistic description of the point-contact geometry.

In Fig. 3 we have plotted the measured temperature dependence of a $\mathrm{Cu}$ point-contact resistarce. In the same figure we have also given as a dashed line the resistance of a plece of bulk wire from which the sharp needle of the point contact was fabricated. For a relative comparison, the scaling of the two curves in Fig. 3 has been done in such a way that the curves fall together at low temperatures and have the same slope at higher temperatures. The overall dependence on temperature is similar for the point-contact resistance and the bulk resistance, but the observed difference is characteristic for our experiments on point contacts and bulk materıal. An explanation for this difference in the tempcrature dependence of the resistance could lie in the fact, that the two parts of the point contact have not ween faoricated from the same starting riaterial. The deviations in the temperature dependent resisitivity, due to diffexent puritıes of samples are known as Deviations of Mathiessen's Rule (DMR), which are snaller than the resiaual resistivity at low temperatures ${ }^{12}$. In our case the difference in the two curves at higher temperatures is approximately two times the residual resistance of the bulk wire and cannot likely be described $1 n$ terms of DMR. One part of the Cu point contact is a single crystal which might yield a different spectrum for $\alpha^{2} F_{p}$ compared with a polycrystaline sample. However, in pressure-type point-contact 


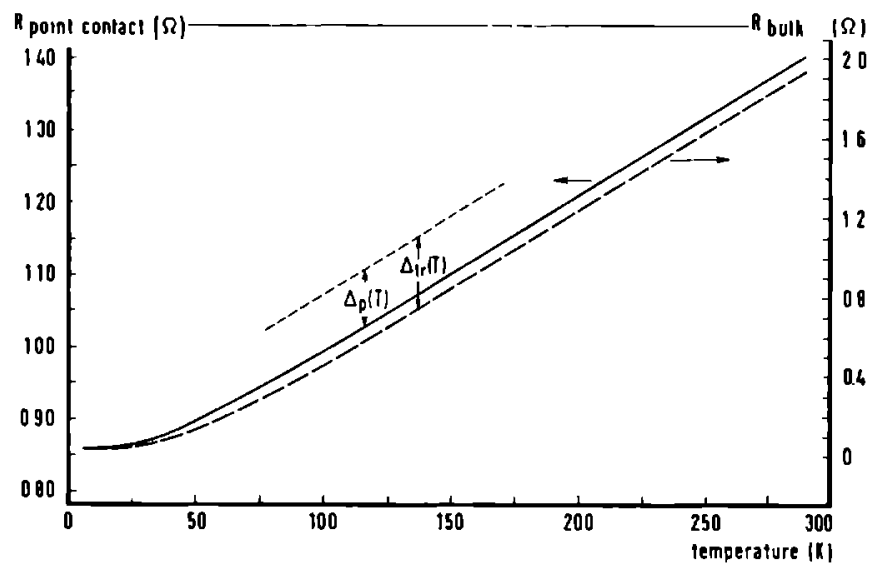

Fig. 3 : Point-contact resistance as a function of the temperature. For comparison the dashed line represents the resistance of a bulk-wire (spear-part of the point contact). The values $\Delta_{p}(T)$ and $\Delta_{t r}(T)$ correspond to the difference between the measured resistance and the straight line through $R(T=0)$ parallel to the high temperature limit of the resistance. 
experiments on $\mathrm{Cu}$ no strong dependence was observed in the measured spectra for different crystalline orlentations of the point contact ${ }^{2,13}$. We therefore conclude that the difference as shown in Fig. 3 might be due to the efficiency functions for the electron-phonon interaction in the bulk resistivity and the point-contact resıstance.

To show the influence of the efficiency function more clearly we will analyse Eqs. (5) and (6) for the resıstance of a point contact and a bulk sample. We define a function $\Delta(T)=T+[R(0)-R(T)] /[\mathrm{dR} / \mathrm{dT}]$, denoted as $\Delta_{p}(T)$ if $R$ is the resistance of the point contact, and as $\Delta_{t r}(T)$ if $R$ is the resistance of the bulk material. The function $\Delta(T)$ corresponds with the difference between the measured resistance and the straight line $\left(R(T)=T\left(\frac{d R}{d T}\right)_{T=\infty}\right)$ parallel to the resistance at high temperatures. The experimental results in Fig. 3 indicate that $\delta\{\Delta(T)\}=\Delta_{p}(T)-\Delta_{t r}(T)<0$. It follows from Eqs. (5) and (6), that $\Delta(T)=T-T[P(T)-Q(T)] /[P(T)+Q(T)]$ or, approximately

$$
\Delta(T) \approx 2 T Q(T) / P(T)
$$

for the case $(Q / P) \ll 1$, where

$$
\begin{aligned}
& Q(T)=\int d \omega \alpha^{2} F(\omega) q\left(\omega / k_{B} T\right) ; q(x)=\frac{x^{2} \operatorname{ch} x-x \operatorname{sh} x}{\operatorname{sh}^{3} x} \\
& P(T)=\int d \omega \alpha^{2} F(\omega) p\left(\omega / k_{B} T\right) ; p(x)=\frac{x^{2} \operatorname{ch} x}{\operatorname{sh}^{3} x}
\end{aligned}
$$

For the function $\alpha^{2} F$ in the definitions of $Q(T)$ and $P(T)$ we take $\alpha^{2} F_{p}$ for the function $\Delta_{p}(T)$ and $\alpha^{2} F_{t r}$ for the function $\Delta_{t r}(T)$. The ratio $(Q / P)$ 
decreases roughly like $(1 / T)$ and appears to be quite small compared to unity. Eq. (11) is useful for comparing the differences between the transport efficlencles for point-contact and bulk resistances. For this purpose the difference $\delta\{\Delta(T)\}=\Delta_{p}(T)-\Lambda_{t r}(T)$ can be related to the difference $\delta\left\{\alpha^{2} F(\omega)\right\}=\alpha^{2} F_{p}(\omega)-\alpha^{2} F_{t r}(\omega)$ in the electron-phonon intaraction functions

$$
\frac{\delta\{\Delta(T)\}}{\Delta(T)}=\frac{\delta Q}{Q}-\frac{\delta P}{P}=\int d \omega \delta\left\{\alpha^{2} F(\omega)\right\}\left[\frac{q(\omega / k T)}{Q(T)}-\frac{P(\omega / k \Gamma)}{P(T)}\right] .
$$

The right hand side of Eq. (13) vanishes if one substıtutes $\delta\left\{\alpha^{2} \mathrm{~F}\right\} \rightarrow$ const. $\alpha^{2} F$, by virtue of the defining equations (12). Consequently, the factor of the integrand between brackets on the right hand side of Eq. (13) must have at least one zero at $\omega_{0}\left(C<\omega_{0}<\omega_{D}\right)$ because $\alpha^{2} F \geqslant 0$. A number of conclusions may be drawn from the experimental fact that $\delta[\Delta(\mathrm{T})\}<0$. For instance, it is not possible that the function $\alpha^{2} F_{p}$ is shifted to higher frequencies compared with $\alpha^{2} F_{t r^{\prime}}$ such that $\alpha^{2} F_{p}<\alpha^{2} F_{\text {tr }}$ at $\omega<\omega_{0}$ and $\alpha^{2} F_{p}>\alpha^{2} F_{t r}$ at $\omega>\omega_{\circ}$. Because of the normalisation $\int d \omega \alpha^{2} F_{P}(\omega) / \omega=$ $\int d \omega \alpha^{2} F_{t r}(\omega) / \omega$ lt 15 not allowed that the difference between $\alpha^{2} F_{P}$ and $\alpha^{2} F_{t r}$ will be positıve or negative for all frequencies. We hence conclude that the function $\alpha^{2} F_{p}$ is snifted to lower frequencies compared with $\alpha^{2} F_{t r}$, such that $\alpha^{2} F_{P}>\alpha^{2} F_{\text {tr }}$ at $\omega<\omega_{0}$ and $\alpha^{2} F_{P}<\alpha^{2} F_{\text {tr }}$ at $\omega>\omega_{0}$. We want to compare this result for the electron-phonon interaction functions $\alpha^{2} F_{p}$ and $\alpha^{2} F_{t r}$ with the behaviour of the efficiency functions of the scattering mechanısm at small phonon frequencies. For normal processes the efficlency functions $\eta(\theta)$ and $(1-\cos \theta)$ behave both 1 lke $\omega^{2}$ for small angle scattexing at low frequencies, and therefore no difference is expected between $\alpha^{2} F_{P}$ and $\alpha^{2} F_{t r}$. Umklapp scatterıng however is much more dominant in the noble metals and particularly important in the point-contact case because of the 
singularity in the function $\eta(0)$ at $\theta=\pi$. Considering umklapp scattering as the main contribution to the electron-phonon interaction function, we find that $(1-\cos \theta) \propto \operatorname{const}$. and $n(\theta) \times \omega^{-\frac{1}{2}}$. According to these arguments the $w$-power will be smaller for ${ }^{2} F_{p}$ than for $\alpha^{2} F_{t r}$, which yield a stronger signal of $\alpha^{2} F_{p}$ at low energies. This is in agreement with the result, deduced from the experimentally measured temperature dependence of a pointcontact resistance and that of bulk material resistivity.

\section{Conclusions.}

Polnt-contact experiments were performed betweer 5 and $300 \mathrm{~K}$. The measured $d^{2} v / d I^{2}$-curves of $c u$ point contacts showed thermal broadening of the structure, caused by the electron-phonon interaction, upon increasıng the temperature. A thermal welgnt function, obtalned from a theoretical analysis, fits the experimental result very well. The measured resistance of $\mathrm{Cu}$ point contacts revealed interesting bohaviour as a function of the temperature, which rescmbled the resistivity of bulk material. In a relative comparison one observes that the resistance of a point contact increases more rapidly than the resistance of bulk materlal. We concluded that the relative difference could be reduced to differences in the electron-phonon interaction $\alpha^{2} F$ fCr a point contact geometry $\left(\alpha^{2} F_{P}\right)$ and bulk material $\left(\alpha^{2} F_{t r}\right)$. On the basis of simple arguments, which include that umklapp processes are dominant over normal processes in noble metals,

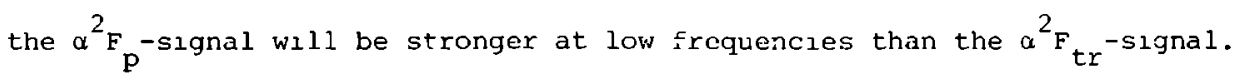
As the weight function in Eqs. (5) and (6) dominates at low frequencles, the difference in the $\alpha^{2}$ F-signals is in agreement with experimental observation. Note however that a more detalled analysis is needed to support this conclusion, for instance because of the non-sphericity of the Fermi- 
sphere in copper. These problems are similar to the calculation of the temperature dependent resistivity. It is of interest to determine the power law of the temperature dependence of the point-contact resistance at low temperatures to study the relevant scattering processes in a point-contact geometry, in analogy to experimental work on the temperature dependence of the resistivity in bulk material.

Acknowledgement.

Part of this work has been supported by the "stıchting voor Fundamenteel Onderzoex der Materıe" (FOM) with financial support of the "Nederlandse Organısatıe voor Zuıver Wetenschappelıjk Onderzoek" (zwo). 
1. I.K. Yanson, Zh. Eksp. Teor. Fız. 66, 1035 (1974) [ Sov. Phys. - JETP 39, 506 (1974) ]; I.K. Yanson and I.O. Kul 1k, J. Phys. (Parıs) 39, C6-1564 (1978), and references theren.

2. A.G.M. Jansen, F.M. Mueller and P. Wyder, Phys. Rev. B16, 1325 (1977) and Science 199,1037 (1978).

3. I.O. Kulık, A.N. Omel'yanchuk and R.I. Shekhter, Fiz. Nızk. Temp. 3 , 1543 (1977) [Sov. J. Low Temp. Phys. 3, 740 (1978)].

4. A.P. van Gelder, Solıd State Commun. 25, 1097 (1978).

5. Yu.V. Sharvin, Zh. Eksp. Teor. F1z. 48,984 (196') I Sov. Phys. - JETP $21,655(1965)]$.

6. A.P. van Gelder, to be published.

7. B. Hayran and J.P. Carbotte, J. Phys, F 2, 915 (1972).

8. J. Lambe and R.C. Jaklevıc, Phys. Rev. 165, 821 (1968).

9. J.G. Adler and J.E. Jackson, Rev. Sc1. Instr. 31, 1049 (1966).

10. A.P. van Gelder, A.G.M. Jansen, S. Strāssler and P. Wyder, J. Phys. (Par1s) 39, C6-602 (1978).

11. J.C. Maxwell, A Treatise on Flectricity and Magnetisn, 1891 (Dover, New York, 1954).

12. J. Bass, Adv. Phys. 21, 431 (1972).

13. I.K. Yanson and G. Batrak, Zh. Eksp. Teor. Fiz. Pis'ma Red. 27 , 212 (1978) [ JETP Lett. 27, 197 (1378) ]. 
Chapter VIII : APPLICATION OF POINT-CONTACT SPECTROSCOPY IN METALS TO THE KONDO PROBLEM.

\section{ABSTRACT}

A general relationship between the characteristic structure in the electrical resistance of a point contact between two metals and the dynamics of the scatterers is used to discuss new experimental results on Kondo systems. The method allows to measure directly the energy dependence of the relaxation time of the conduction electrons. 
The problem of point contact spectroscopy with respect to the electron-phonon interaction has recently been discussed in several papers ${ }^{1}$. Here we want to show that the method can be generalized to obtain information on the dynamics of arbitrary scatterers. In particular we have applied the method of point contact spectroscopy for the first time to Kondo systems.

The expression for a current I flowing through a point contact is given by $I=\iint d x d y J_{z}(x, y, 0)$, where the integral is taken over the surface of the orifice. The current density J is given by (unit volume) $\underline{I}(\underline{r})=2 e \sum_{\underline{k}} \underline{v}_{\underline{k}} f_{\underline{k}}(\underline{r})$. Here, $\underline{v}_{\underline{k}}$ is the band velocity with wave vector $\underline{k}$ and the electron distribution $f_{\underline{k}}(\underline{r})$ satisfies the Boltzmann equation

$$
\left.\underline{(\underline{\mathrm{v}} \cdot \underline{\nabla}-\underline{\underline{r}}}+\frac{e}{h} \underline{E} \cdot \underline{\nabla} \underline{\underline{k}}\right) f_{\underline{k}}=\left.\frac{\partial \underline{f}}{\partial t}\right|_{\operatorname{coll}} .
$$

The electric field $E$ must be determined from Poissons equation

$$
-\Delta \phi=\underline{e} \underline{\nabla} \cdot \underline{E}=-\frac{2 e \underline{E_{0}}}{\sum_{\underline{k}}} f_{\underline{k}}(\underline{r}) .
$$

The following boundary conditions must be satisfied:

$$
\mathrm{f}_{\underline{k}}(z \rightarrow \pm \infty)=\mathrm{f}_{T}(\varepsilon(\underline{k})), \phi(z \rightarrow \pm \infty)= \pm \mathrm{eV} / 2
$$

$f_{T}$ is the equilibrium Fermi function and $V$ the applied voltage over the point contact. $\mathrm{c}(\underline{\mathrm{k}})$ is the electronic energy measured relative to the chemical potential $\mu$. The collision term in Fq. (1) for scattering of the electron from an arbitrary target (phonons, spins, etc.) is 


$$
-\left.\frac{\partial f}{\partial t}\right|_{\operatorname{coll}}=\sum_{\underline{k}{ }^{\prime}} \Gamma\left(\underline{k}, \underline{k}^{\prime}\right) f_{\underline{k}}\left(1-f_{\underline{k}},\right)-\Gamma\left(\underline{k}^{\prime}, \underline{k}\right) \underline{f}_{\underline{k}},\left(1-\underline{f}_{\underline{k}}\right)
$$

where

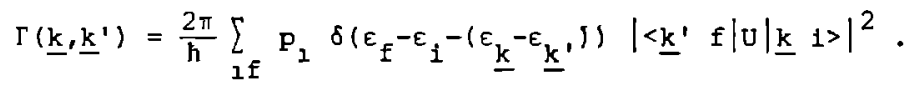

Here $p_{1}$ is the probablinty for the target to be in the state 1 and $E_{1}$ and $\varepsilon_{f}$ are the initial and final energies of the target. $U$ describes the interaction between scattering electrons and target.

of particular interest is the voltage dependence of the point contact resistance $R(V)=d V / d I$ because it contains information about the scatterer and the interaction. This information can be obtained easily only in the limit where the mean free path is comparable or larger than the diameter $d$ of the orifice. Therefore it is appropriate to solve Eqs. (1) and (2) by iteration with respect to the collision terms. In zeroth order one obtalns ${ }^{1} I^{(0)}=e_{0}\left\langle\left|v_{k z}\right|\right\rangle_{0} v$, where $D_{0}$ is the area of the orifice and $\langle\cdots\rangle_{\omega}=\sum_{\underline{k}} \cdots \delta\left(\varepsilon_{\underline{k}}-w\right)$. In first order the result 15 $I^{(1)}=I^{(0)}+\Delta I^{(1)}$, where $\frac{k}{\mathrm{k}}$

$$
\Delta I^{(1)}=-2 e \int_{0}^{e V} d \omega \int_{0}^{\omega} d \omega^{\prime}\left\langle\left\langle\Gamma\left(\underline{k}, \underline{k}^{\prime}\right) K\left(\underline{k}, \underline{k}^{\prime}\right)\right\rangle_{\omega^{\prime}} \omega^{\prime} .\right.
$$

The weight factor $K\left(\underline{k}, \underline{k}^{\prime}\right)$ in Eq. (6) is the common volume defined by the two cylinders constructed with the boundary line of the orifice and whose walls are // to $\underline{v}_{\underline{k}}$ or $\underline{v}_{\underline{k}}$, respectively. In the case of independent localized isotropic scatterers the change in the resistance is given by $R=R_{0}+\Delta R$, where $R_{0}=V / I(0)$ and 


$$
\Delta \mathrm{R}=\mathrm{C} \int_{0}^{\mathrm{eV}} \mathrm{d} \omega^{\prime} \Gamma\left(\mathrm{eV}, \omega^{\prime}\right) \mathrm{N}(\mathrm{eV}) \mathrm{N}\left(\omega^{\prime}\right)
$$

Here $\mathrm{C}=\mathrm{R}_{0}^{2} 2 \mathrm{e}^{2}\left\langle\left\langle\mathrm{k}\left(\underline{\mathrm{k}}, \underline{k}^{\prime}\right)\right\rangle_{0}\right\rangle_{0}$, and $\mathrm{N}(\omega)=\langle 1\rangle_{\omega^{\prime}} /\langle 1\rangle_{0}$ is the normalized density of states. The relaxation time $\tau$ for an electronic state with energy eV above the Fermi surface is

$$
\tau^{-1}(\mathrm{eV})=\sum_{\underline{k}^{\prime}} \Gamma\left(\underline{\mathbf{k}}, \underline{\mathbf{k}}^{\prime}\right) \equiv\langle 1\rangle \int_{0}^{\mathrm{eV}} \Gamma\left(\mathrm{eV}, \omega^{\prime}\right) \mathrm{N}\left(\omega^{\prime}\right) \mathrm{d} \omega^{\prime} .
$$

For a dilute system the resistance change linear in the target concentration is simply related to the relaxation time by $(\mathrm{N}(u)=1)$

$$
\Delta \mathrm{R}=\frac{\mathrm{C}}{\langle 1\rangle_{0}} \mathrm{~T}^{-1}(\mathrm{eV})
$$

It follows from $\mathrm{Eq}$. (9) that it is possible to measure directly the energy dependence of the relaxation time $\tau(\omega)$ by measuring the voltage dependence of the point contact resistance $R(V)$. We have applied this finding to Kondo systems.

For Kondo systems, the relaxation time has been evaluated theoretically in several Refs. ${ }^{2}$. In particular, Hamann ${ }^{2}$ gives for $e v>k_{B} T$ :

$$
\tau^{-1}(e V)=\frac{c}{\pi<1\rangle_{0}}\left\{1-\ln \left(\frac{e V}{k_{B} T_{K}}\right)\left[n^{2}\left(\frac{e V}{k_{B} T_{K}}\right)+s(s+1) \pi^{2}\right]^{-\frac{1}{2}}\right\}
$$

where $T_{K}$ is the characteristic Kondo temperature of the dilute alloy and $c$ the concentration of magnetic impurities with spin s. 
We have investigated the current-voltage characteristics of point contacts of two systems with a Kondo temperature above and below the measuring temperature of the helıum bath $(T=1.5 \mathrm{~K})$ : CuFe and AuMn, wlth $\mathrm{T}_{\mathrm{K}}$ resp. $\sim 30 \mathrm{~K}$ and $\sim \mathrm{O} \mathrm{K}$ and with nominal magnetic impurity concentrations between 0.03 and 1.0 at. ${ }^{3}$. The point contacts were of the pressure type and could be adjusted by pressing a sharply etched wire against a flat surface, botn parts fabricated from the same starting material. The derivatives dV/aI of the contacts were measured using phase sensitive detection. The modulation voltage was in the range of 100-300 $\mathrm{V}$ and didn't exceed the expected thermal broadening of a few $k_{B} T$. With a superconducting coll a magnetic field up to $30 \mathrm{kG}$ could be applied perpendicular to the contact surface.

In all point contacts of the unvestigated alloy systems the measured second derivative $d^{2} v / d I^{2}$ showed structures at voltages corresponding to the phonon frequencies, as explained for the pure metal case in terms of the electron-phonon interaction ${ }^{1}$ (see the inserts in the figures 1 and 2 ). At voltages lower than the phonon frequencies we observed maxima in the resistance dV/dI, which were practically symmetric around zero voltage. Because of the observed phonon structure in the point contact spectra our observations in the metal-metal contacts are essentially different from the spectroscopy with metal-oxide-metal tunneljunctions, where the exchange coupling between the electrons and the magnetic impurities in the oxıde layer results in a minimum (as compared with the maximum in the point contact case) in the resistance around zero voltage, as firstly recognized experimentally by wyatt ${ }^{4}$.

In Fig. 1 the measured resistance of a AuMn point contact is shown as function of the applied voltage. Because of the small kondo temperature of the AdMn system $\left(\left|\operatorname{lnT} \mathrm{T}_{K}\right|>>1\right)$ Eq. (10) leads to $\tau^{-1}(\mathrm{eV})$ a 


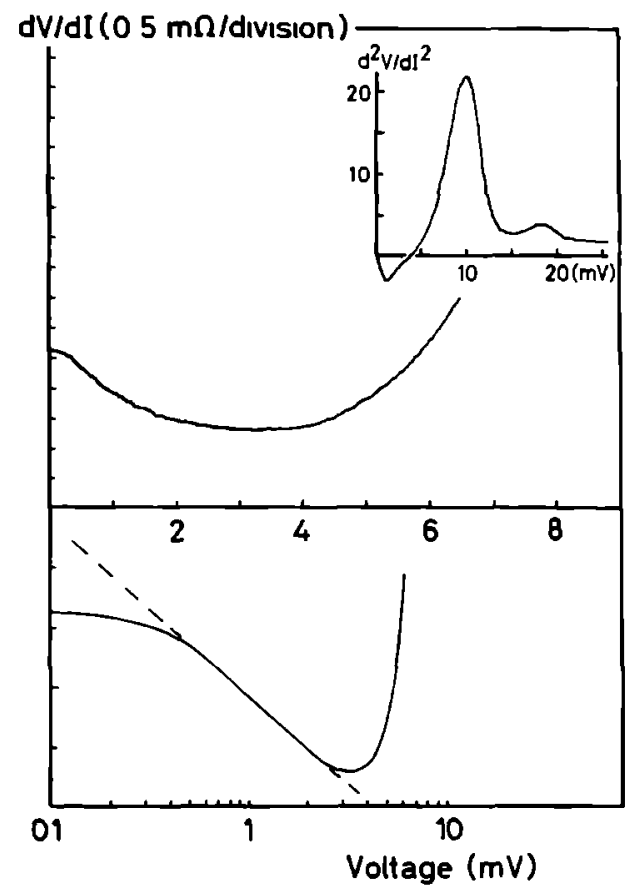

Fig. 1 : Differential resistance dV/dI of a Au-0.03 at. 8 Mn point contact as a function of the applied voltage on a linear and a logarıthmıc scale $(\mathrm{R}=2.1 \Omega ; \mathrm{T}=1.5 \mathrm{~K})$. The dashed ine gives the theoretically expected logarithmic behaviour. The inserted $d^{2} v / d I^{2}$-curve indicates the well known typical point contact behaviour with the phonon peaks for Au at 10 and $18 \mathrm{mV}$. 


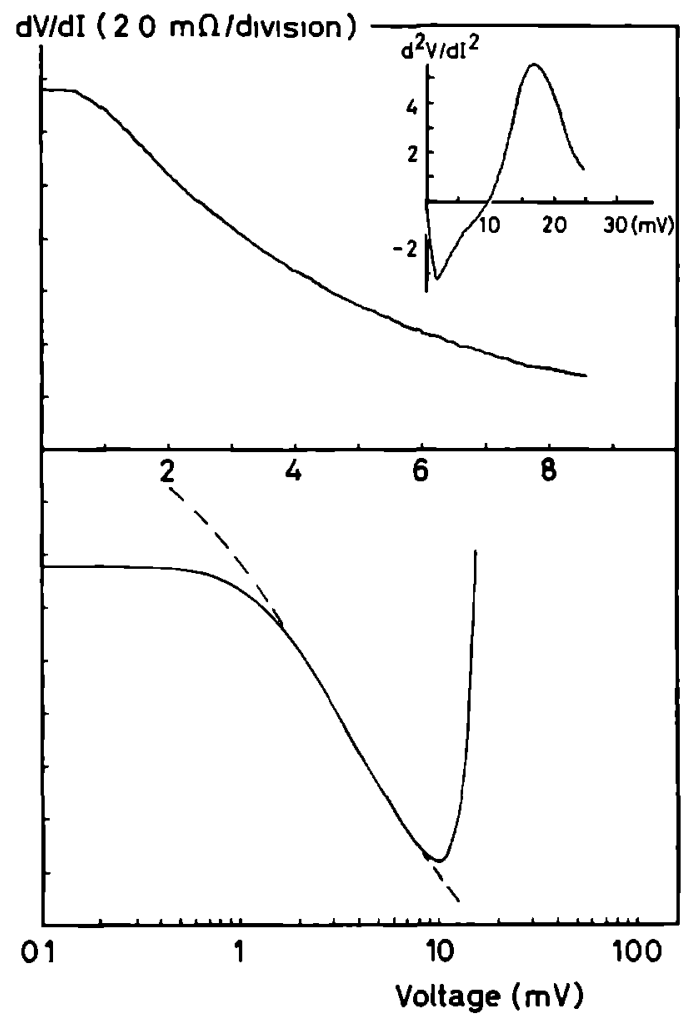

Fig. 2 : Differential resistance dV/dI of a Cu-0.1 at. 8 Fe point contact as function of the applied voltage on a linear and a logarıthmıc scale $(R=1.2 \Omega ; T=1.5 \mathrm{~K})$. The dashed curve is the fitted Hamann-function with $\mathrm{k}_{\mathrm{B}} \mathrm{T}_{\mathrm{K}}=3.5 \mathrm{mV}$ and $\mathrm{s}=0.5$. The inserted $\mathrm{d}^{2} \mathrm{v} / \mathrm{dI}^{2}$-curve indicates the well known typlcal polnt contact behaviour with the phonon peak for $\mathrm{Cu}$ at $17 \mathrm{mV}$. 
$\left[1-2 \ln (e V) / \ln \left(k_{B} T_{K}\right)\right]$. As shown in $F_{1 g}$. 1 on a log-scale the resistance behaves over a limited range of $\mathrm{V}$ like logV around $1 \mathrm{mV}$. At higher voltages the resistance increases due to the electron-phonon interaction. At lower voltages the deviation is probably due to thermal averaging around zero voltage and ordering effects. Note that ordering effects have also been observed in measurements of the temperature dependence of the electrical resistivity in bulk Au samples with Mn concentrations $\geq 0.02$ at. $z^{5}$. In Fig. 2 the measured voltage dependence of a CuFe point contact resistance is given on a linear scale and a log-scale. Between 2 and $7 \mathrm{mv}$ a logv behaviour is seen for the CuFe syster as expected according to formula (10) at voltages corresponding to the kondo temperature $(30 \mathrm{~K} \sim 2.6 \mathrm{mV})$. For snaller voltages the contact resistance becomes nearly constant and a fit to the Hamann-function isn't successful. Note that using the Hamann-formula the obtalned temperature dependence of the electrical resistivity is similar to the energy dependence of the scattering rate ${ }^{2}$. Below the kondo temperature the same deviation has been measured in the electrical resistivity of the bulk material (see Fig. 18 in Ref. 6). The point contact resistance becomes a constant at applied voltages which are roughly twice the corresponding temperatures in the electrical resistivity measurements.

By applying a magnetic field the resistance maximum at zero voltage splits up in two maxima for the case of AuMn, as shown in Fig. 3. For the CuFe case the magnetic fields up to $30 \mathrm{~kg}$ lead only to a rounding off of the flat resistance maximum, without significartly influencing the amplitude of the change in resistance. Assuming that the "Kondo field" $\mathrm{H}_{\mathrm{K}}=\mathrm{k}_{\mathrm{B}} \mathrm{T}_{\mathrm{K}} / \mu_{\mathrm{B}}$ acts in a similar way as the temperature, the quantitatıve difference for the two systems can be understood. A similar magnetic field dependence ras been observed in bulk resistivity measurements as 


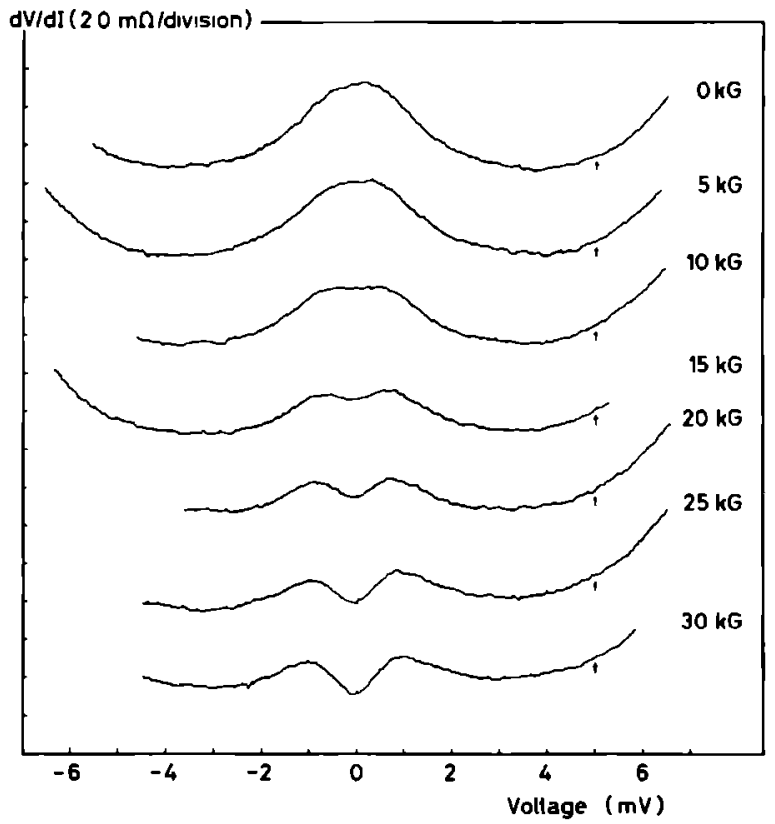

Fig. 3 : Magnetic field dependence of the differential resistance $\mathrm{dV} / \mathrm{dI}$ of a Au-0.1 at. $\mathrm{m} \mathrm{Mn}$ point contact as function of the applied voltage $(R=2.5 \Omega ; T=1.5 \mathrm{~K})$. The curves are shifted with respect to the reference point indicated by ( ) . 
function of temperature for dilute alloys with a low and a high Kondo temperature ${ }^{7}$. With increasing $M_{n}$ concentration in the Au samples the curves of the point contact resistances as function of voltage resembled the pattern plotted in Fig. 3 upon increasing the magnetic field, probably due to ordering or, equivalently, internal fields. This has also been seen experimentally in bulk resistivity measurements ${ }^{5}$.

In the case of CuFe point contacts we also observed minima in the resistance around zero voltage in some cases. It should be noted however that zero blas anomalies (resistance minima and maxima and no structure at all) have also been observed in the pure metal point contacts in an irreproducible way. It is of interest to establish experimentally whether these anomalies in the pure metal case are due to magnetic inpurities. Furthermore, it is not unlikely for the used nominal concentrations of $\mathrm{Fe}$ in $\mathrm{Cu}$, that the impurities are not distributed homogeneously over the sample.

We conclude from our observations on the voltage and magnetic field dependence of the resistance of point contacts in Kordo systems to have extended the usefulness of the point contact spectroscopy to obtain the energy dependence of the scattering rate of the conduction electrons in a kondo system.

We are most thankful to Dr. B. Knook from the Kamerlingh Onnes Laboratorium in Lelden for preparing the Kondo samples and very grateful to Prof. J. Bass for his stimulating interest in a early stage of this study. Part of this work has been supported by the Stichting FoM. 


\section{$\underline{\text { References }}$}

1. I.K. Yanson, Zh. Eksp. Teor. Fiz. 66, 1035 (1974) [ Sov. Phys. - JETP 39, 506 (1974) ] ; A.G.M. Jansen, F.M. Mueller and P. Wyder, Phys. Rev. B 16, 1325 (1977); A.G.M. Jansen, F.M. Mueller and P. Wyder, Science 199, 1037 (1978); I.K. Yanson and I.O. Kulik, J. Phys. (Paris) 39, C6-1564 (1978) and references therein.

2. Magnetisn, vol. V, edited by H. Suhl (Academic Press, New York, 1973); D.R. Hamann, Phys. Rev. 158, 570 (1967).

3. The samples were prepared by Dr. B. Knook at the Kamerlingh Onnes Laboratorium in Leiden and are registrated under KoL 78131-132 and KOL $78134-137$.

4. A.F.G. Wyatt, Phys. Rev. Lett. 13,40 (1964).

5. J.W. Loram, T.E. Whall and P.J. Ford, Phys. Rev. B 3.953 (1971).

6. J.W. Loram, T.E. Whall and P.J. Ford, Phys. Rev. B 2,857 (1970).

7. P. Monod, Phys, Rev. Lett. 19, 1113 (1967). 

This thesis deals with an experimental investigation of interesting deviations from Ohm's law which have been observed at low temperatures in metallic point contacts, if the linear dimension of the contact becomes comparable to the mean free path of the electrons. This geometry allows to have an electric field within a metal in the region of the contact due to an applied roltage. The conduction electrons are accelerated in this electric field and come back in equilibrium via collisions with elementary excitations (1.e. phonons) in the metal. Because these inelastic scattering processes depend strongly on the energy of the accelerated electrons, the current-voltage characteristic of a point contact is nonlinear. The observed non-linearity allows an energy resolved spectral analysis of the inelastic collisions of the electrons in a metal using point contacts. The measured second derıvatıve $d^{2} v / d I^{2}$ of metallic point contacts reveal structures at applied voltages which are in agreement with the phonon frequencles of the metal and allow a direct measurement of the energy dependence of Ellashbergselectron-phonon interaction parameter $\alpha^{2} F$.

After a general introduction to the spectroscopic method, chapter II gives a survey on the theoretical and experımental work done in pointcontact spectroscopy up to now. The full non-linear Boltzmann equation for the current through a metallic constriction is solved, using an iterative procedure with respect to the inelastic collision term in the transport equation. It is found that the measured resistance of a point contact as a function of the applied voltage, is proportional to the inverse of the energy-dependent scattering time of the electrons in a metal, forming the contact. For the electron-phonon interaction, this means that the second derivative signal is proportional to the $\alpha^{2} F$-spectrum, taking into account a transport efficiency function due to the specific geometry. The experimental techniques used for polnt-contact spectroscopy are discussed. The data for varzous metals give detalled and novel results on the energy dependence of the electron-phonon interaction, especially for the normal (1.e. non-superconducting) metals.

The following chapters contain experiments dealing with certain aspects of polnt-contact spectroscopy. Experiments with pressure-type point contacts of the noble metals (chapter III and IV) give a remarkable inte- 
resting $\alpha^{2}$ F-spectrum which shows that the coupling of the electrons is stronger with transverse phonons than with longltudinal phonons. It is shown that the observed background signal, on which the $\alpha^{2}$ F-spectrum 15 thought to be superimposed, can not be explained by double phonon processes only, and non-equilibrium phonons have to be 1 nvolved (chapter $v$ ). In chapter VII experiments on the temperature dependence of the spectroscoplc method are described, and one finds an analogy between the temperature dependence of the contact resistance at zero voltage and that of the bulk resıstivity,except for small alfferences arısing from the different geometrical transport efficiencles in the two cases. In chapter VIII, experimental evidence is given for the detection of the energy dependent exchange interaction between electrons and paramagnetic impurities in point-contact experiments with magnetically dilute alloys (Kondo systems). 
Dit proefschrift behandelt een experımenteel onderzoek van interessante afwijkıngen van de wet van Ohn, die bıj lage temperaturen $z 1 j n$ waargenomen in puntkontakten tussen metalen, waarvan de afmetingen vergelıjkbaar zıjn met de vrıje weglengte van de elektronen. Door het aanleggen van een spanning over het puntkontakt ontstaat bij het kontakt een elektrisch veld in het metal. De geleldingselektronen worden versneld in dit elektrische veld en kunnen weer in evenwicht komen door middel van botsıngen met elementaire excitaties (b.v. fononen) in het metaal. Ondat deze ınelastısche verstroolıngsprocessen sterk afhangen van de energie van de versnelde elektronen, is het verband tussen stroom en spannıng in een puntkontakt niet linealr. De waargenomen afwıjkıng van de wet van Ohm maakt het mogelıjk de inelastısche botsingen van de elektronen in een metaal als funktie van de energie van de elektronen spektraal te analyseren met behulp van puntkontakten. In de tweede afgeleıde $d^{2} v / d I^{2}$, gemeten aan een puntkontakt tussen metalen, is een struktuur te zlen bij aangelegde spannıngen, die overeenkomen met de frequenties van de fononen in het metaal en de Ellashberg functie $\alpha^{2} F$ voor de elektron-fonon interaktie valt hlerult direkt te bepalen. Na een algemene Inlelding op de methode, geeft hoofdstuk II een overzlcht van het theoretische en experimentele werk, dat tot op heden aan puntkontaktspektroskopie gedaan 1s. De Boltzmann vergelıjking voor het transport van elektronen door een puntkontakt wordt opgelost door gebrulk te maken van een iteratieve methode ten opzichte van de inelastısche verstroolingsterm in de transportvergelıjking. Men komt tot de conclusie dat de weerstand van een puntkontakt, die als funktıe van de aangelegde spannıng wordt gemeten, omgekeerd evenredig is met de verstroolıngstıjd van de elektronen in het metaal, die van de energie van de elektronen afhangt. Dit heeft voor de elektron-fonon interaktıe tot gevolg dat het signaal van de tweede afgeleıde evenredig is met het $\alpha^{2}$ F-spektrum, daarbıJ moet rekening worden gehouden met de effectivitelt van het transport van elektronen in de specifleke geometrıe van een puntkontakt. De experımen tele technleken, die gebrulkt worden voor puntkontakten, worden besproken. De experimentele resultaten voor de verschlllende metalen laten gedetallleerd zien hoe de elektron-fonon interaktıe van de energie afhangt; met name voor de normale (d.w.z. niet-supergeleldende) metalen kan deze af- 
hankelıjkheld voor het eerst experımenteel bepaald worden.

De volgende hoofdstukken bevatten experımenten die bepaalde aspecten van puntkontaktspektroskople behandelen. Experımenten met puntkontakten van de edele metalen, (hoofdstuk III en IV) geven een opmerkelıjk $\alpha^{2} F-$ spektrum te zien, waarvoor geldt dat de koppeling van de elektronen sterker is met transversale fononen dan met longltudinale fononen. De waargenomen achtergrond in het gemeten signaal kan niet alleen verklaard worden door processen, waarbıj de elektronen aan twee fononen verstroold worden; in een analyse van dit achtergrondsignaal moet rekening worden gehouden met het felt dat de elektronen niet in evenwicht zijn (hoofdstuk V). In hoofdstuk VII worden puntkontakten als funktie van de temperatuur bestudeerd, en wat betreft de afhankelıjkneld van de temperatuur zıet men een analoog gedrag voor de puntkontaktweerstand en voor de weerstand van bulk materıaal, behoudens kleine verschillen in de effectiviteit van de verstrooling van de elektronen.

In hoofdstuk VIII laten experımenten aan puntkontakten met kondosystemen zien dat ook de wisselwerking tussen elektronen en paramagnetische verontreınıgingen in een metaal met deze methoden bestudeerd kunnen worden. 


\author{
A.G.M. Jansen
}

Geboren : 3 februarı 1952 te Breda.

1964 - 1970 : Middelbare schooloplelding aan het Onze Lleve Vrouwe Lyceum te Breda; afgesloten met het eindexamen Gymasium B.

1970 - 1977 : Unıversitalre studıe natuurkunde aan de Facultelt der W1skunde en Natuurwetenschappen van de Katholieke Unlversitelt Nijmegen; afgesloten met het doctoraalexamen natuurkunde met als hoofdvak experimentele natuurkunde.

1977 - 1980 : Promotıeonderzoek op de afdelıng Experımentele Natuurkunde 4, Faculteit der Wiskunde en Natuurwetenschappen, Katholıeke Unıversıtelt Nijmegen, in het kader van een FOM-beleıdsrulinte projekt onder leıding van Prof. P. Wyder. 

Met behulp van foto-thermische lonisatie-spektroskople kunnen donoren en acceptoren in halfgeleiders afzonderlıjk worden waargenomen. Uıt het teken van de bijdrage van de minderheldsonzulverheden aan de electrische geleiding kunnen conclusies worden getrokken over de relevante recombunatie processen. H.W.H.M. Jongbloets, Proefschrift, N1jmegen 1980.

II

Bij de interpretatie van de resultaten van magnetısche metingen aan een koper(I)-koper(II) complex is de door Schugar en medewerkers genntroduceerde antiferromagnetische wisselwerking voor een groot deel toe te schrijven aan verzadiglngseffecten ten gevolge van de grootte van het aangelegde veld. De temperatuurafhankelıjkheld van hun metıngen blj lage temperaturen wordt beter beschreven door deze hypothetische antıferromagnetische wisselwerking nul te kiezen, waarbij echter wel de schaalfactor moet worden aangepast. H.J. Schugar et al., Inorg. Chem. 19, 543 (1980).

III

De levensduur van geexclteerde electronen blj cyclotron resonantie in halfgeleiders kan worden bepaald ust een gecombineerde meting van de absorptie van stralıng ten gevolge van cyclotron resonantie en van de corresponderende veranderıng van het electrische geleidingsvermogen voor halfgeleideıs met parabolısche banden en als functie van het ingestraalde vermogen. J.C. Maan, Proefschrift, Nıjmegen 1979.

Door de gelıjkrıchtende werkıng van puntkontakten tussen metalen te bestuderen als functie van de ingestraalde frekwentıe ( $>$ THz) kunnen de relaxatıetıjden van de electron-fonon wisselwerkıng in een metaal bepaald worden. 
Indien de waargenomen verhoging van de kritische stroom en temperatuur voor een supergeleider onder Invloed van mlcrogolf straling toe te schrifven is aan een verhoging van de orderparameter van de supergeleider kan dit nader worden onderzocht door een bepaling van het kritische veld.

T.M. Klapwijk, Proefschrift, Delft 1977.

VI

Verschillen tussen de met behulp van puntkontakten gemeten spektra van de functie $\alpha^{2} F$, die worden toegeschreven aan anisotropie effecten ten gevolge van de verschillende kristalorientaties in de buurt van het kontakt, kunnen worden geanalyseerd door de spektra opgebouwd te denken ult 3 functles voor de verschillende kristalrichtingen.

VII

De uitgever verhoogt de service aan de lezers van zlfn boeken door, zoals in het verleden gebruikelijk, bij zijn boeken weer een antwoordkaart of soortgelijke bladwijzer te voegen.

VIII

Het kraken van woningen werkt de maatschappelifke integratle van de politie tegen.

IX

Uit het feit dat in de identiteitsbewijzen van de gehuwde vrouw het bifvoegsel "echtgenote van" staat en niet in die van de gehuwde man, blifkt dat de formele gelifkstelling van man en vrouw bif de betreffende overheldsInstanties nog niet ten volle is doorgedrongen.
A.G.M. Jansen
N1jmegen, 27 maart, 1980. 

